\title{
Early-time light curves of Type Ib/c supernovae from the SDSS-II Supernova Survey ${ }^{\star \star \star \star}$
}

\author{
F. Taddia ${ }^{1}$, J. Sollerman ${ }^{1}$, G. Leloudas ${ }^{2,3}$, M. D. Stritzinger ${ }^{4}$, S. Valenti ${ }^{5,6}$, L. Galbany ${ }^{7,8}$, R. Kessler ${ }^{9,10}$, \\ D. P. Schneider ${ }^{11,12}$, and J. C. Wheeler ${ }^{13}$
}

${ }^{1}$ Department of Astronomy, The Oskar Klein Centre, Stockholm University, AlbaNova, 10691 Stockholm, Sweden e-mail: francesco.taddia@astro.su.se

2 The Oskar Klein Centre, Department of Physics, Stockholm University, AlbaNova, 10691 Stockholm, Sweden

3 Dark Cosmology Centre, Niels Bohr Institute, University of Copenhagen, Juliane Maries Vej 30, 2100 Copenhagen, Denmark

${ }^{4}$ Department of Physics and Astronomy, Aarhus University, Ny Munkegade 120, 8000 Aarhus C, Denmark

5 Las Cumbres Observatory Global Telescope Network, 6740 Cortona Dr., Suite 102, Goleta, CA 93117, USA

${ }^{6}$ Department of Physics, University of California, Santa Barbara, Broida Hall, Mail Code 9530, Santa Barbara, CA 93106-9530, USA

7 Millennium Institute of Astrophysics, Universidad de Chile, Casilla 36-D Santiago, Chile

8 Departamento de Astronomía, Universidad de Chile, Casilla 36-D Santiago, Chile

9 Department of Astronomy and Astrophysics, The University of Chicago, 5640 South Ellis Avenue, Chicago, IL 60637, USA

${ }_{10}$ Kavli Institute for Cosmological Physics, The University of Chicago, 5640 South Ellis Avenue, Chicago, IL 60637, USA

11 Department of Astronomy and Astrophysics, The Pennsylvania State University, University Park, PA 16802, USA

12 Institute for Gravitation and the Cosmos, The Pennsylvania State University, University Park, PA 16802, USA

13 Department of Astronomy, University of Texas at Austin, Austin, TX 78712, USA

Received 31 March 2014 / Accepted 20 October 2014

\section{ABSTRACT}

Context. Type $\mathrm{Ib} / \mathrm{c}$ supernovae $(\mathrm{SNe} \mathrm{Ib} / \mathrm{c}$ ) have been investigated in several single-object studies; however, there is still a paucity of works concerning larger, homogeneous samples of these hydrogen-poor transients, in particular regarding the premaximum phase of their light curves.

Aims. In this paper we present and analyze the early-time optical light curves (LCs, ugriz) of $20 \mathrm{SNe}$ Ib/c from the Sloan Digital Sky Survey (SDSS) SN survey II, aiming to study their observational and physical properties, as well as to derive their progenitor parameters.

Methods. High-cadence, multiband LCs are fitted with a functional model and the best-fit parameters are compared among the SN types. Bolometric LCs (BLCs) are constructed for the entire sample. We also computed the black-body (BB) temperature $\left(T_{\mathrm{BB}}\right)$ and photospheric radius $\left(R_{\mathrm{ph}}\right)$ evolution for each $\mathrm{SN}$ via $\mathrm{BB}$ fits on the spectral energy distributions. In addition, the bolometric properties are compared to both hydrodynamical and analytical model expectations.

Results. Complementing our sample with literature data, we find that SNe Ic and Ic-BL (broad-line) have shorter rise times than those of SNe Ib and IIb. The decline rate parameter, $\Delta m_{15}$, is similar among the different subtypes. SNe Ic appear brighter and bluer than $\mathrm{SNe} \mathrm{Ib}$, but this difference vanishes if we consider host galaxy extinction corrections based on colors. Templates for SN Ib/c LCs are presented. Our SNe have typical $T_{\mathrm{BB}}$ of $\sim 10000 \mathrm{~K}$ at the peak and $R_{\mathrm{ph}}$ of $\sim 10^{15} \mathrm{~cm}$. Analysis of the BLCs of SNe Ib and Ic gives typical ejecta masses $M_{\mathrm{ej}} \approx 3.6-5.7 M_{\odot}$, energies $E_{\mathrm{K}} \approx 1.5-1.7 \times 10^{51} \mathrm{erg}$, and $M\left({ }^{56} \mathrm{Ni}\right) \approx 0.3 M_{\odot}$. Higher values for $E_{\mathrm{K}}$ and $M\left({ }^{56} \mathrm{Ni}\right)$ are estimated for SNe Ic-BL $\left(M_{\mathrm{ej}} \approx 5.4 M_{\odot}, E_{\mathrm{K}} \approx 10.7 \times 10^{51} \mathrm{erg}, M\left({ }^{56} \mathrm{Ni}\right) \approx 1.1 M_{\odot}\right)$. For the majority of SNe Ic and Ic-BL, we can put strong limits $(<2-4$ days) on the duration of the expected early-time plateau. Less stringent limits can be placed on the duration of the plateau for the sample of SNe Ib. In the single case of SN Ib 2006lc, a >5.9 days plateau seems to be detected. The rising part of the BLCs is reproduced by power laws with index $<2$. For two events (SN 2005hm and SN 2007qx), we find signatures of a possible shock break-out cooling tail.

Conclusions. Based on the limits for the plateau length and on the slow rise of the BLCs, we find that in most of our SNe Ic and Ic-BL the ${ }^{56} \mathrm{Ni}$ is mixed out to the outer layers, suggesting that $\mathrm{SN}$ Ic progenitors are de facto helium poor. The derived progenitor parameters $\left({ }^{56} \mathrm{Ni}, E_{\mathrm{K}}, M_{\mathrm{ej}}\right)$ are consistent with previous works.

Key words. supernovae: general

\section{Introduction}

Recent supernova ( $\mathrm{SN}$ ) surveys are dramatically changing the landscape in observational $\mathrm{SN}$ research. Not only are we finding

* Figures 1, 7 and Tables 1-7 are available in electronic form at http://www . aanda.org

$\star \star$ Light curve templates are only available at the CDS via anonymous ftp to cdsarc.u-strasbg. fr (130.79.128.5) or via http://cdsarc.u-strasbg.fr/viz-bin/qcat?]/A+A/574/A60 many new kinds of explosions (e.g., super-luminous SNe, Gal-Yam 2012), but we are also able to study them over a wider parameter space in time and wavelength. One particular aspect in modern time-domain astronomy is the possibility to systematically discover stellar explosions at an extremely early stage. These detections have hitherto been done rather serendipitously, often for nearby objects.

In this paper we focus on the early phases (premaximum light) of Type $\mathrm{Ib} / \mathrm{c} \mathrm{SNe}(\mathrm{SNe} \mathrm{Ib} / \mathrm{c})$. These classes of $\mathrm{SNe}$ lack 
hydrogen lines in their spectra. Strong helium lines characterize the spectra of $\mathrm{SNe} \mathrm{Ib}$, whereas $\mathrm{SNe}$ Ic are also heliumpoor (e.g., Filippenko 1997). A subclass of SNe Ic (Ic-broad line, Ic-BL) shows high expansion velocities $\left(\sim 3 \times 10^{4} \mathrm{~km} \mathrm{~s}^{-1}\right.$ a few days after explosion). These $\mathrm{SNe}$ are often associated with long-duration gamma-ray bursts (GRBs, e.g., Galama et al. 1998). Transitional events called SNe IIb present hydrogen lines in their early spectra, but at the maximum light, these objects exhibit the characteristic helium lines of SNe Ib (e.g., SN 1993J, Filippenko et al. 1993; SN 2011dh, Ergon et al. 2014).

The sample of $\mathrm{SNe} \mathrm{Ib/c} \mathrm{in} \mathrm{the} \mathrm{literature} \mathrm{is} \mathrm{still} \mathrm{relatively}$ small ${ }^{1}$. Drout et al. (2011) present a compilation of $V$ - and $R$-band light curves for 25 stripped-envelope (SE) SNe. There are also several studies that present optical (and sometimes near infrared) light curves and spectra for single events. Recently, Cano (2013) has compiled and modeled the photometric data for $61 \mathrm{SNe} \mathrm{Ib/c} \mathrm{and} \mathrm{Ic-BL} \mathrm{in} \mathrm{the} \mathrm{literature.} \mathrm{Richardson} \mathrm{et} \mathrm{al.}$ (2014) present absolute $B$-band peak magnitudes for $69 \mathrm{SNe} \mathrm{Ib/c}$ and IIb.

The early emission from the resulting $\mathrm{SNe}$ is powered by both the shock energy and energy from the radioactive decay of ${ }^{56} \mathrm{Ni}$ (see, e.g., Piro \& Nakar 2013, hereafter PN13, for a pedagogical introduction). The first electromagnetic signal from a SN Ib/c is the actual shock breakout (e.g., SN 2008D, Soderberg et al. 2008; although see Mazzali et al. 2008 for a different interpretation). In the optical regime this feature is observable only in the cooling phase, and is typically short-lived for compact stars. It has been predicted that a plateau phase, which could be rather faint, follows this stage (Dessart et al. 2011; Bersten et al. 2013; PN13), until the radioactive decay energy starts the light curve rise in earnest. $\mathrm{SNe} \mathrm{Ib} / \mathrm{c}$ exhibit light curves that rise to peak in just $\sim 15-20$ days. The current information on the rise times of these explosions was summarized by Valenti et al. (2011). Their compilation of $16 \mathrm{SNe}$ suggested that there is a diversity in rise times and that helium-rich (IIb/Ib) SNe may have longer rise times, because of either ${ }^{56} \mathrm{Ni}$ mixed to outer layers (PN13) and/or higher ejecta masses.

The two leading scenarios for the nature of SN Ib/c progenitor stars are those that either consider a single, massive ( $M_{\text {ZAMS }} \gtrsim 25 M_{\odot}$, Massey 2003) Wolf-Rayet (WR) star, which sheds its hydrogen envelope through a prevalent line-driven wind or, alternatively, a binary system where a less massive progenitor loses its hydrogen-rich envelope also via tidal stripping from a companion. Studies of the environments of $\mathrm{SNe} \mathrm{Ib} / \mathrm{c}$ (Modjaz et al. 2011; Kuncarayakti et al. 2013) could suggest differences in local metallicity between $\mathrm{SNe} \mathrm{Ib}$ and Ic, with the former showing lower metallicities. This result would indicate that metal-line winds play an important role in distinguishing $\mathrm{SN}$ Ib and Ic progenitors, favoring the single-star scenario. However, this result is not confirmed by other works (Anderson et al. 2010; Leloudas et al. 2011; Sanders et al. 2012). More and better data is needed to differentiate a single-star from a binary origin on the basis of different metallicities. Considerations of SN rates (Smith et al. 2011) and age constraints on SN locations (Leloudas et al. 2011) do indicate that at least some SNe Ib/c originate in binary systems. For SNe IIb there is direct evidence that their progenitors belong to binary systems (e.g., SNe 1993J, Maund et al. 2004). Recently, light-curve modeling of SN Ib iPTF13bvn (Fremling et al. 2014) and SN IIb 2011dh (Ergon et al. 2014, 2015) (both $\mathrm{SNe}$ have pre-explosion detection of

\footnotetext{
1 During the refereeing process, the light curves of 64 SE-SNe and optical spectra of 73 SE-SNe were released by Bianco et al. (2014) and Modjaz et al. (2014), respectively.
}

the progenitor star) revealed low-mass progenitor stars for these transients, implying that they belong to binary systems.

In this paper we present the light curves of a sample of $20 \mathrm{SNe} \mathrm{Ib} / \mathrm{c}$ in their photospheric phase obtained by the Sloan Digital Sky Survey (SDSS-II) SN survey (York et al. 2000; Sako et al. 2008, 2014; Frieman et al. 2008). The properties of the sample are quantified and compared among the spectral subclasses. Progenitor properties are estimated from the bolometric light curves that we build from the multiband (ugriz) data. The cadence of the SDSS-II SN survey allows the discovery of $\mathrm{SNe}$ soon after explosion, thereby acting as a forerunner to ongoing and future surveys like $\mathrm{iPTF}^{2}$ and $\mathrm{ZTF}^{3}$ (intermediate Palomar Transient Factory and Zwicky Transient Facility; Kulkarni 2012), where the rationale is to increase the cadence, thereby systematically discovering and monitoring transients at very early stages. Surveys like PTF have in fact already discovered a few events soon after explosion, such as SN 2011dh (Arcavi et al. 2011). The detection of $\mathrm{SNe} \mathrm{Ib} / \mathrm{c}$ in the early stages is important for probing the explosion physics by investigating of the ${ }^{56} \mathrm{Ni}$ mixing, as well as the properties of the progenitors from constraints on the stellar radius. These discoveries have focused recent theoretical interest toward the early light curves of SE CC SNe (PN13, Dessart et al. 2011), and our aim here is to complement these works by exploring what can be done with a larger observational sample of such discoveries, in particular when multiband data are available.

This paper is organized as follows. In Sect. 2 we introduce the dataset from the SDSS-II SN survey used in this paper and describe the observations and reductions. The analysis of these light curves is given in Sect. 3, and then a more general discussion is provided in Sect. 4. Our conclusions and future outlook are provided in Sect. 5.

\section{Data from SDSS-II}

The data used in this paper were obtained by SDSS-II and released by Sako et al. $(2014)^{4}$. The SDSS-II SN survey operated three fall campaigns in 2005, 2006, and 2007 using the $2.5 \mathrm{~m}$ telescope (Gunn et al. 2006) at Apache Point Observatory to scan $300 \mathrm{deg}^{2}$ of sky. The cadence was in principle as often as every second night, although practical conditions (e.g., weather) often decreased that somewhat (see below).

Each photometric observation consisted of nearly simultaneous $55 \mathrm{~s}$ exposures in each of the five ugriz filters (Fukugita et al. 1996) using the wide-field SDSS CCD camera (Gunn et al. 1998). High-quality light curves were obtained on a photometric system (Ivezić et al. 2004) calibrated to an uncertainty of $1 \%$ (Mosher et al. 2012). Details on the photometric measurements are provided by Holtzman et al. (2008) and Sako et al. (2014).

The primary aim of the SN survey was to discover and characterize several hundred SNe Ia at intermediate redshifts, in order to fill in the SN redshift desert, located between the lowand high $z$, samples for cosmological studies. Results based on the first season of SN Ia data were presented in Kessler et al. (2009), Sollerman et al. (2009), and Lampeitl et al. (2010), while Betoule et al. (2014) make use of the intermediate-redshift $(0.05<z<0.4)$ SN Ia data collected during all three SDSS-II seasons. The SDSS-II SN survey provided a large and uniform

\footnotetext{
2 http://www.ptf.caltech.edu/iptf

http://www.ptf.caltech.edu/ztf

4 Data are available at:

http: //sdssdp62. fnal.gov/sdsssn/DataRelease/index .html
} 
sample of SNe, which allowed a detailed study of their observational properties. The rise times of SNe Ia were discussed in detail by Hayden et al. (2010a,b). The rolling search strategy and the high cadence of the SDSS-II SN survey assured that many of the SDSS SNe have well-defined, densely sampled light curves, even on the rise portion. The data also include pre-explosion flux limits.

Although CC SNe were not the primary aim of the SDSS-II SN survey, many such events were also discovered, monitored, and spectroscopically confirmed (Sako et al. 2008, 2014). A sample of nearby SNe IIP was published by D'Andrea et al. (2010), and some individual peculiar CC SNe have also been reported previously (e.g., Leloudas et al. 2012). Leloudas et al. (2011) discuss the spectroscopic classification of several of these objects in relation to a study of their host galaxies. Here we focus on a set of 20 SDSS-II SNe discovered between 2005 and 2007 in untargeted galaxies, which were spectroscopically confirmed as $\mathrm{SNe} \mathrm{Ib} / \mathrm{c}$. The basic information on these $\mathrm{SNe}$ are provided in Table 1.

The classification of each SN (Table 1, 4th column) is based on spectra taken by the SDSS-II Supernova Survey team that were cross-correlated to SN spectral templates (Zheng et al. 2008). After the SDSS-II SN survey classification, some of the SNe were typed again by Leloudas et al. (2011), and we adopt the latter classification ${ }^{5}$. Our sample consists of nine $\mathrm{SNe} \mathrm{Ib}$, six SNe Ic, and five SNe Ic-BL. SN 2005em, classified as a SN Ic by Zheng et al. (2008), was typed as a SN IIb by Morrell et al. (2005) and is not included in our sample. We reclassified SN 14475 and SN 2006nx as SNe Ic-BL based on the best SNID (Blondin \& Tonry 2007) fits for the known host galaxy redshifts. (The 3 other SNe Ic-BL were classified by Zheng et al. 2008.) Selected spectra of our targets are displayed in Fig. 1. Many of our SNe have multiple spectra (Sako et al. 2014), and a spectral $\log$ is provided in Table 2. The spectra shown in Fig. 1 were selected based on their quality and phase (their epochs are marked by vertical red lines in Fig. 4 and by a star in Table 2), aiming to illustrate the spectral differences among the three types (Ib, Ic Ic-BL). We chose to only display spectra obtained around or after $r$-band maximum (which was possible in all but a few cases) in order to clearly show which SNe are helium-rich and which are helium-poor. As already noted by Leloudas et al. (2011), the detailed subclassification of SNe Ib and Ic that was made from the SDSS-II spectra is sometimes problematic.

The SN redshifts, usually obtained from the host galaxy spectra (Zheng et al. 2008), range from 0.016 (SN 2006lc) to 0.264 (SN 2005fk). On average, the SNe Ic and SNe Ib discovered by SDSS-II were located at similar redshifts $\left(\bar{z}_{\text {Ic }}=0.064 \pm\right.$ $\left.0.023, \bar{z}_{\mathrm{Ib}}=0.057 \pm 0.052\right)$, whereas SNe Ic-BL were typically found at somewhat higher redshifts $\left(\overline{\bar{z}}_{\mathrm{Ic}-\mathrm{BL}}=0.156 \pm 0.063\right)$.

Luminosity distances were computed from the redshifts assuming WMAP (5years) cosmological parameters $\left(H_{0}=\right.$ $70.5 \mathrm{~km} \mathrm{~s}^{-1} \mathrm{Mpc}^{-1}, \Omega_{\mathrm{m}}=0.27, \Omega_{\Lambda}=0.73$, Komatsu et al. 2009), using the cosmology calculator by Wright (2006) via $\mathrm{NED}^{6}$. The visual extinction due to our Galaxy is taken from Schlafly \& Finkbeiner (2011) via NED and is also listed in Table 1.

The reported $g$-band absolute magnitude $\left(M_{g}^{\mathrm{gal}}\right)$ for each host galaxy was obtained through the apparent magnitude listed in

\footnotetext{
5 SN 2006lc is considered an intermediate case between Ib and Ic by Leloudas et al. (2011). Here we include it in the SN Ib sample (as also done in Modjaz et al. 2014), as it shows some helium features, although weak.

6 http://ned.ipac. caltech.edu
}

Hakobyan et al. (2012) or in the SDSS catalog ${ }^{7}$. We K-corrected the galaxy magnitudes using the $g-r$ color of each galaxy ${ }^{8}$ (Chilingarian et al. 2010; Chilingarian \& Zolotukhin 2012). The absolute galaxy magnitudes range from $M_{g}^{\text {gal }}=-15.5$ mag to $M_{g}^{\text {gal }}=-21.2$ mag. The host galaxies of SNe Ic-BL appear slightly fainter $\left(\left\langle M_{g}^{\text {gal }}(I c-B L)\right\rangle=-18.82 \pm 0.43 \mathrm{mag}\right)$ than those of SNe Ib $\left(\left\langle M_{g}^{\mathrm{gal}}(I b)\right\rangle=-19.61 \pm 0.56 \mathrm{mag}\right)$ and Ic $\left(\left\langle M_{g}^{\mathrm{gal}}(I c)\right\rangle=-19.55 \pm 0.58 \mathrm{mag}\right)$.

Light curves for each SN and for each filter (ugriz) are shown in Figs. 2-6. The fluxes are presented in $\mu \mathrm{Jy}$, and the plots also include the pre-explosion epochs, where the fluxes are consistent with zero emission. For each light curve, the temporal axis is stretched by the factor $1 /(1+z)$ in order to correct for time dilation. The times in this paper are all in the rest frame of the supernova unless stated otherwise. In Figs. 2-6, we also indicate the last epoch of non-detection and the first detection. The first detection corresponds to the first epoch on the light curve rise when the $g$ and/or $r$-band flux with its $1 \sigma$ error is not compatible with zero emission. The epochs of the spectra are marked by vertical segments in the $r$-band plot. The first spectral observation occurred before $r$-band maximum for eight out of the $20 \mathrm{SNe}$.

The average observed cadence of the SN photometric observations is $4.4 \pm 0.6$ days. In Fig. 7 (left panel) the distribution for the $r$-band cadence shows that SNe Ib and Ic were observed with similar cadence. The righthand panel of Fig. 7 presents the distribution of the follow-up duration (in $r$ band), showing that these $\mathrm{SNe}$ were observed on average for $51 \pm 27$ days, with a minimum duration of nine days (SN 2005mn) and a maximum of 89 days (SN 2006fe). Therefore most of these $\mathrm{SNe} \mathrm{Ib} / \mathrm{c}$ are observed in their photospheric phase.

The $r$-band light curves (Fig. 4) show that 15 objects were observed both before and after maximum and that 12 of them also have pre-explosion images (15 objects in total have preexplosion images). In summary, this SDSS data set of SNe Ib/c is well-suited to studying these fast transients given its unique high cadence, its multiband coverage and the availability of preexplosion images.

\section{Analysis}

\subsection{Light curve fit}

To quantitatively compare the SN light curves, we performed fits with the phenomenological model used by Bazin et al. (2011). In this model the flux $F(t)$ in each filter is given by

$F(t)=A \frac{\mathrm{e}^{-\left(t-t_{0}\right) / \tau_{\text {fall }}}}{1+\mathrm{e}^{-\left(t-t_{0}\right) / \tau_{\text {rise }}}}+c$.

To illustrate the different parameters that Eq. (1) includes and the light curve properties that we derive and discuss in the next sections, Fig. 8 presents an example of this model fit to the $r$ band light curve of SN $2005 \mathrm{hm}$.

The model is shown by a black solid line, which smoothly fits the flux at each epoch after first detection. In this specific case, we excluded the epoch of first detection from the fit (indicated by a vertical red dashed line), because it may belong to the early shock break-out cooling tail ${ }^{9}$. The last non-detection is denoted by a vertical dashed blue line. The explosion epoch

\footnotetext{
http://skyserver.sdss3.org

8 http://kcor.sai.msu.ru/about/

9 The same was done for the first epoch of SN 2007qx, and the first two epochs of SN 2006lc.
} 

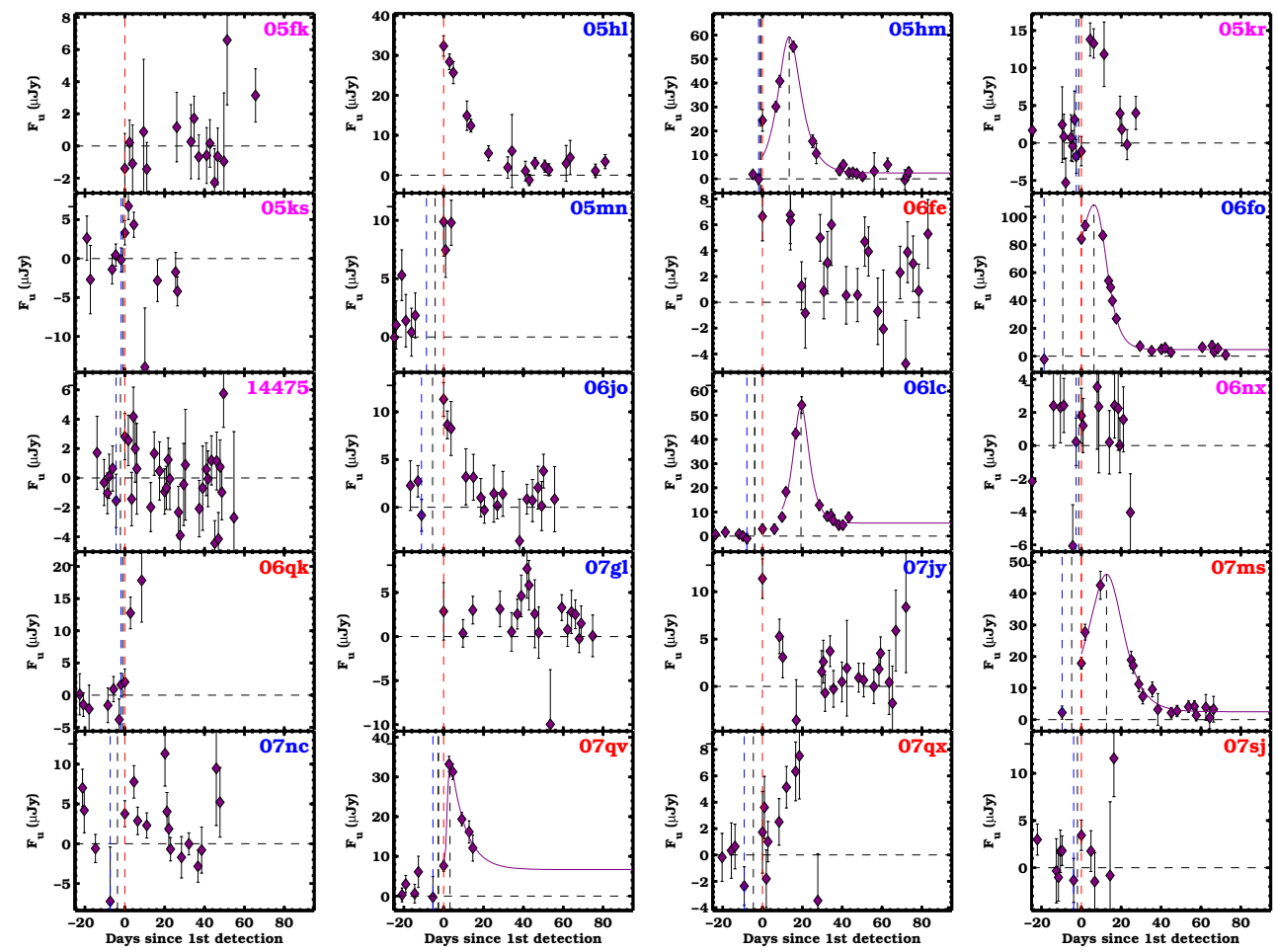

Fig. 2. $u$-band light curves of 20 SDSS SNe Ib, Ic, and Ic-BL. Best fits on the SN light curves observed before peak are shown by a violet solid line. Vertical blue and red dashed lines mark the last non-detection and the first detection epoch. Vertical black dashed lines indicate the derived explosion date and the peak epoch. Light curves are shown after correction for time dilation. Blue, red, and magenta labels correspond to SNe Ib, Ic, and Ic-BL, respectively.

is assumed here to be the mean between the last non-detection and the first detection epochs $\left(t_{\text {expl }}=\frac{1}{2}\left(t_{\text {last non det. }}+t_{1 \text { st det. }}\right)\right)$, and it is shown by a vertical black dashed line in the figure. The uncertainty in this quantity corresponds to half the time interval between those two epochs $\left(\Delta t_{\text {expl }}=\frac{1}{2}\left(t_{\text {1st det. }}-t_{\text {last non det. }}\right)\right)$. The light-curve rise time $t_{\text {rise }}$ (see the double-sided arrow in the top left corner of Fig. 8) is then defined as the time interval between this assumed explosion epoch and the time of the peak $\left(t_{\max }\right.$, see the black dotted vertical line). The epoch of maximum is measured from the fit and is given by $t_{\max }^{k}=t_{0}+\tau_{\text {rise }} \ln \left(\tau_{\text {fall }} / \tau_{\text {rise }}-1\right)$.

The light curve model can be understood as the combination of two main factors: a function (magenta in Fig. 8) with asymptotic values zero (at $t=-\infty$ ) and $\sqrt{A}($ at $t=\infty$ ) that modulates an exponential declining function (green in Fig. 8) with normalization $\sqrt{A}$. The parameter $t_{0}$ represents both the inflection point for the modulating function and the epoch when the exponential function is equal to its normalization. The product of these two factors plus an additional positive constant c (horizontal black dotted line in Fig. 8) reproduce the general light curve shape very well, with a fast rise followed by an exponential decline. The time scales of rise and fall are set by the parameters $\tau_{\text {rise }}$ and $\tau_{\text {fall }}$. The lower the value of $\tau_{\text {rise }}$, the steeper the rise of the light curve (given that all the other parameters are constant). Since at late times (when $t-t_{0} \gg \tau_{\text {rise }}$ ) the model approaches an exponential decline, the late time slope is determined by $\tau_{\text {fall }}$; i.e., the higher the value of $\tau_{\text {fall }}$, the slower the decline of the light curve (given the same normalization parameter). We emphasize that this formula does not have any physical motivation. It is, however, useful to obtain good fits even for poorly sampled data and therefore used to quantify many of the properties of the SDSS-II SE-SN light curves.

The fit was applied to all objects whose maximum was covered by observations. Each fit was made on the epochs following and including discovery. We present the best fit to each light curve with solid, colored lines in Figs. 2-6 and the fit parameters are presented in Table 3. The error on each parameter was estimated by fitting $N=1000$ Monte Carlo simulated light curves ${ }^{10}$ and taking the standard deviation of the resulting $\mathrm{N}$ parameter values. When the parameter errors were considerably larger than the parameters themselves, we considered the best fit to be unreliable and we excluded the results from Table 3. This occurred in a few cases (e.g., in the $z$ band for SNe 2007nc and 2007qv) where the light curves are poorly sampled and/or have large photometric uncertainties. Beside the fit parameters, Table 3 also presents the epoch of maximum light $\left(t_{\max }\right)$, the observed flux at maximum $\left(F_{\max }\right)$, the difference in magnitude between maximum epoch and +15 and -10 days since maximum $\left(\Delta m_{15}\right.$, $\Delta m_{-10}$ ), the rise time $t_{\text {rise }}$ (see Sect. 3.2.2), $t_{\text {expl }}$, the peak apparent magnitude $\left(\mathrm{m}_{\max }\right)$, and the peak absolute magnitude $\left(M_{\max }\right)$. In the following, we discuss these parameters for the different filters and SN types.

\subsection{Light curve properties}

\subsubsection{Peak epoch as a function of the effective wavelength, $t_{\text {max }}\left(\lambda_{\text {eff }}\right)$}

The phenomenological fit to the light curves allows a comparison of the light curve properties of the different events. A first result from the fit is that each SN peaks first in the $u$ band, then in the $g$ band, and successively later in the redder bands. (The $z$ band sometimes peaks at the same time or slightly earlier than the $i$ band.) This systematic property is shown in the top panel

\footnotetext{
${ }^{10}$ For each point of the light curve we produced $N$ normally-distributed flux values, with the central value of the normal distribution equal to the flux at that epoch and $\sigma$ equal to the flux uncertainty.
} 
F. Taddia et al.: Early-time light curves of SDSS-II SNe Ib/c
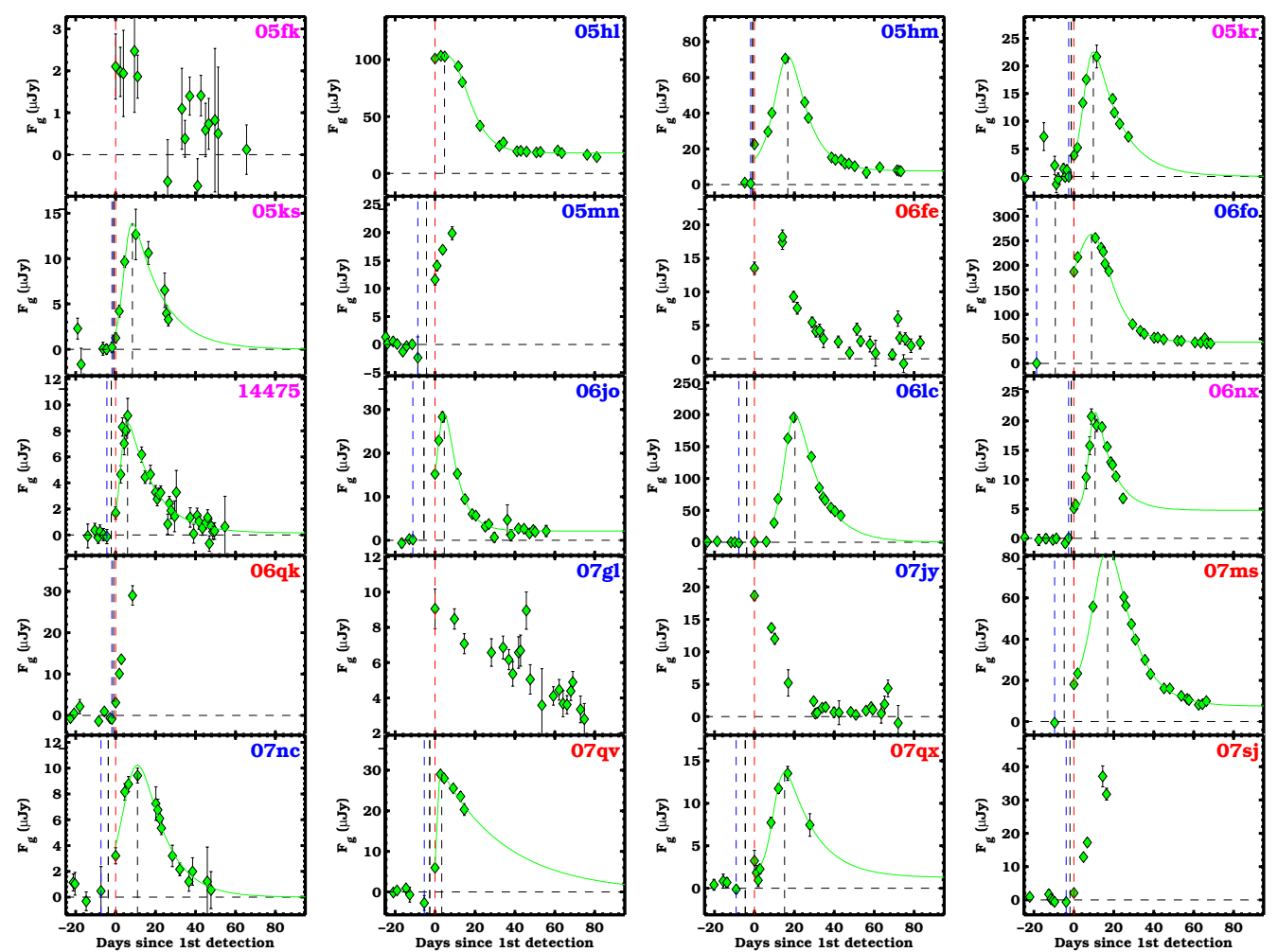

Fig. 3. $g$-band light curves of 20 SDSS SNe Ib, Ic, and Ic-BL. Best fits on the SN light curves observed before the peak are shown by a green solid line. Vertical blue and red dashed lines mark the last non-detection and the first detection epochs. Vertical black dashed lines indicate the derived explosion date and the peak epoch. Light curves are shown after correction for time dilation. Blue, red, and magenta labels correspond to SNe Ib, Ic, and Ic-BL, respectively.
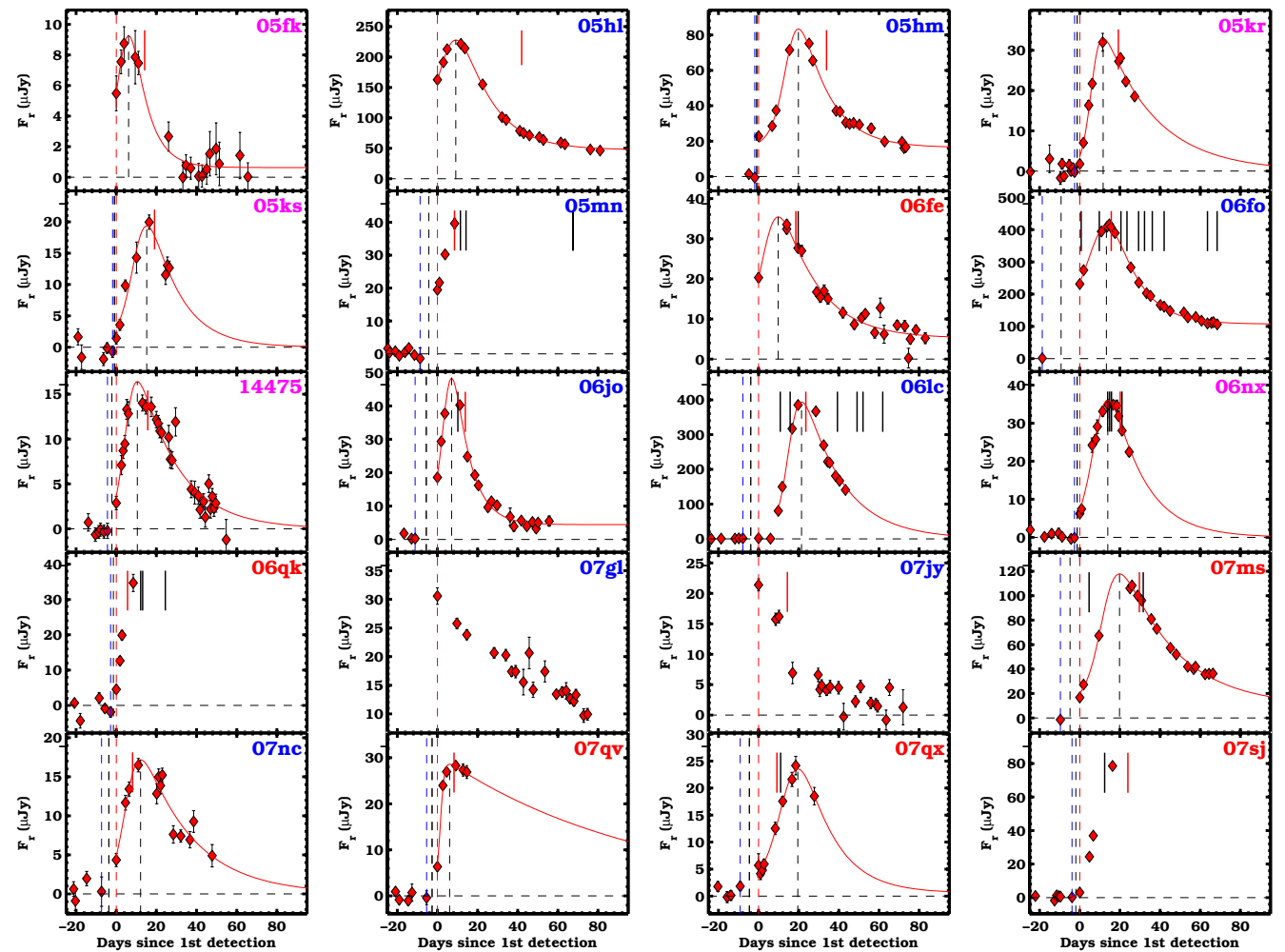

Fig. 4. $r$-band light curves of 20 SDSS SNe Ib, Ic, and Ic-BL. Best fits on the SN light curves observed before the peak are shown by a red solid line. Vertical blue and red dashed lines mark the last non-detection and the first detection epochs. Vertical black dashed lines indicate the derived explosion date and the peak epoch. Black and red vertical segments mark the spectral epochs; in particular, the red ones indicate the epoch of the spectra shown in Fig. 1. Light curves are shown after correction for time dilation. Blue, red, and magenta labels correspond to SNe Ib, Ic, and Ic-BL, respectively. 

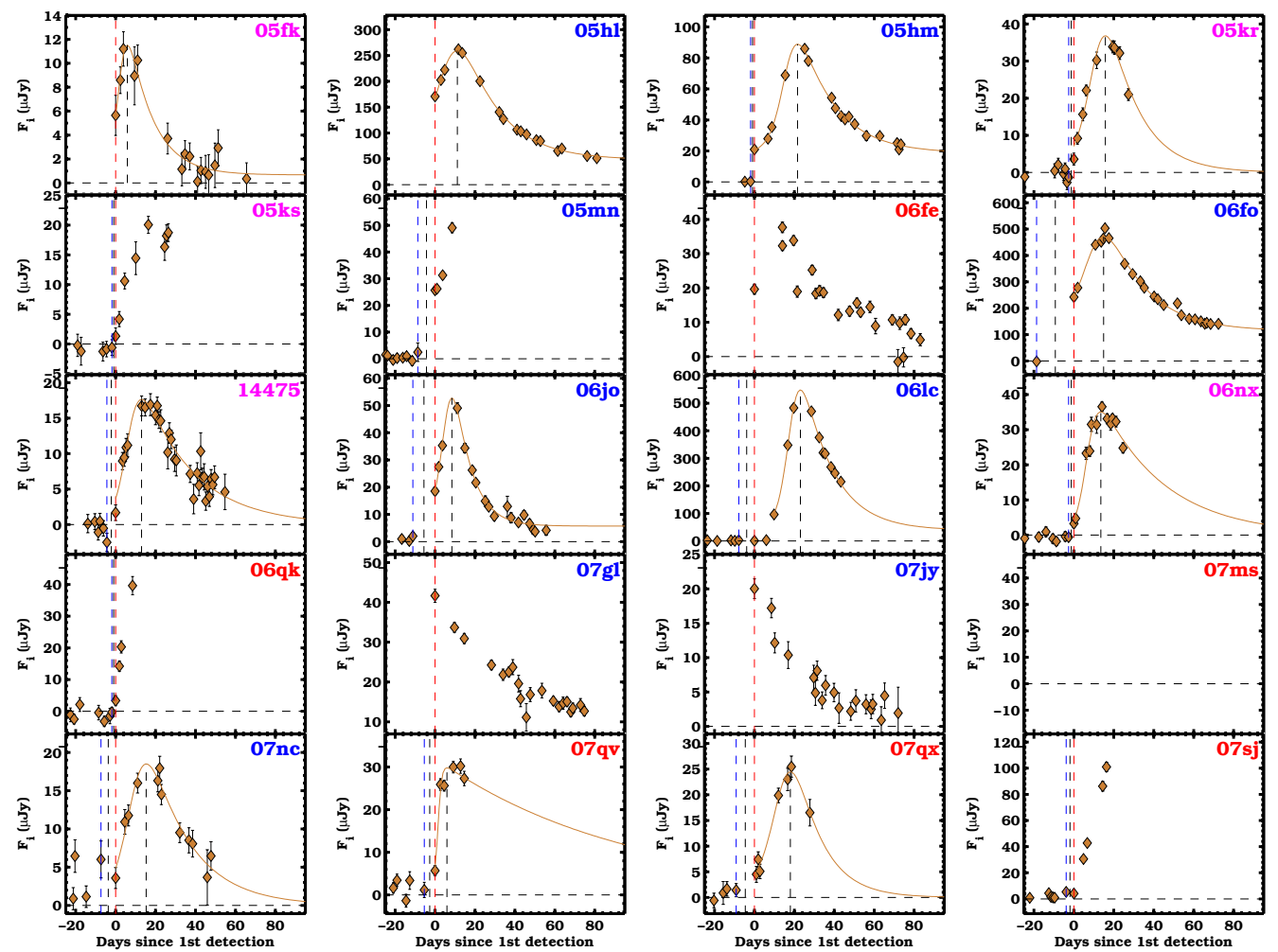

Fig. 5. $i$-band light curves of 20 SDSS SNe Ib, Ic, and Ic-BL. Best fits on the SN light curves observed before peak are shown by a dark yellow solid line. Vertical blue and red dashed lines mark the last non-detection and the first detection epoch. Vertical black dashed lines indicate the derived explosion date and the peak epoch. Light curves are shown after correction for time dilation. Blue, red, and magenta labels correspond to $\mathrm{SNe} \mathrm{Ib}$, Ic, and Ic-BL, respectively.
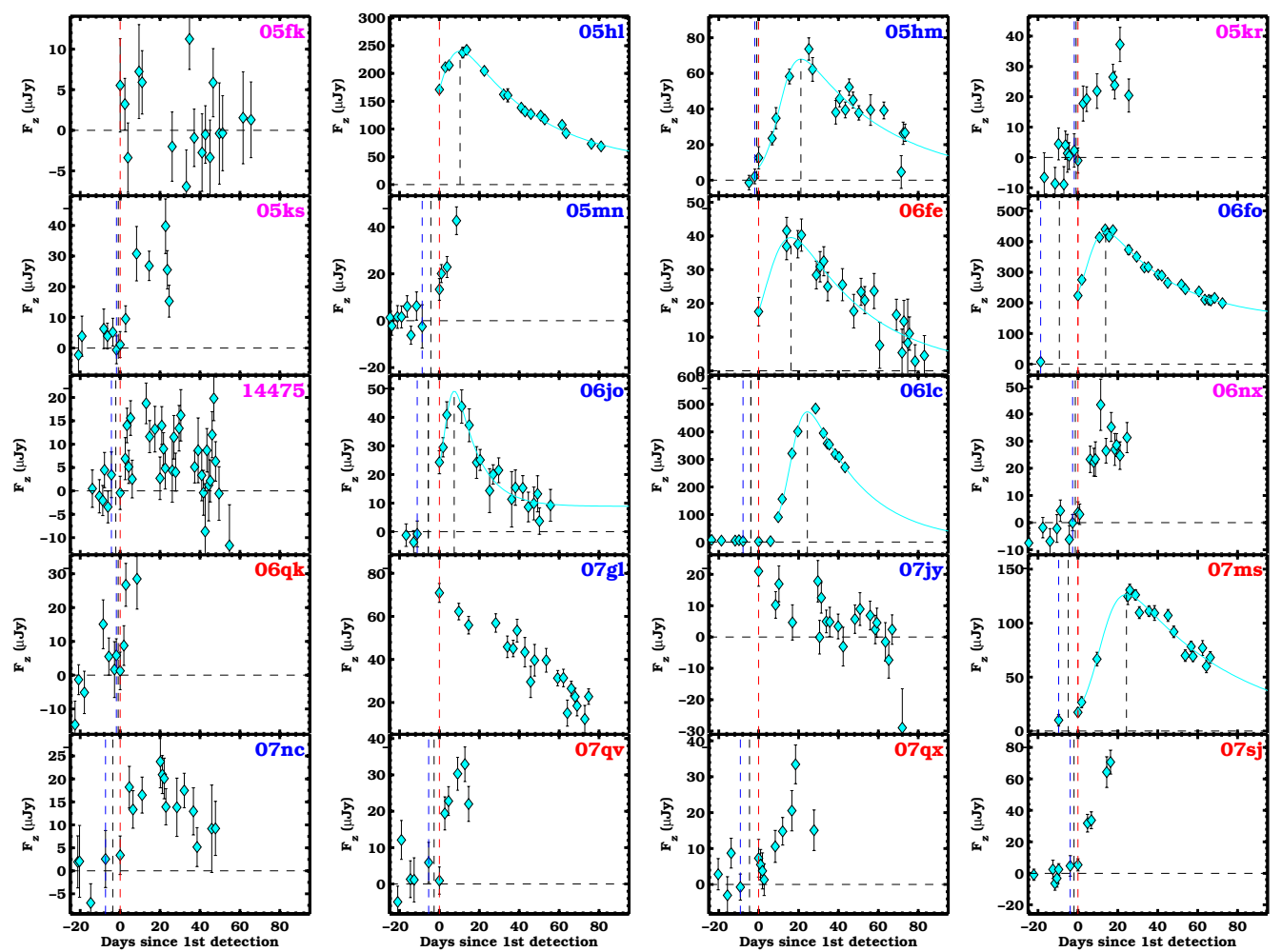

Fig. 6. $z$-band light curves of 20 SDSS SNe Ib, Ic, and Ic-BL. Best fits on the SN light curves observed before peak are shown by a cyan solid line. Vertical blue and red dashed lines mark the last non-detection and the first detection epoch. Vertical black dashed lines indicate the derived explosion date and the peak epoch. Light curves are shown after correction for time dilation. Blue, red, and magenta labels correspond to SNe Ib, Ic, and Ic-BL, respectively. 


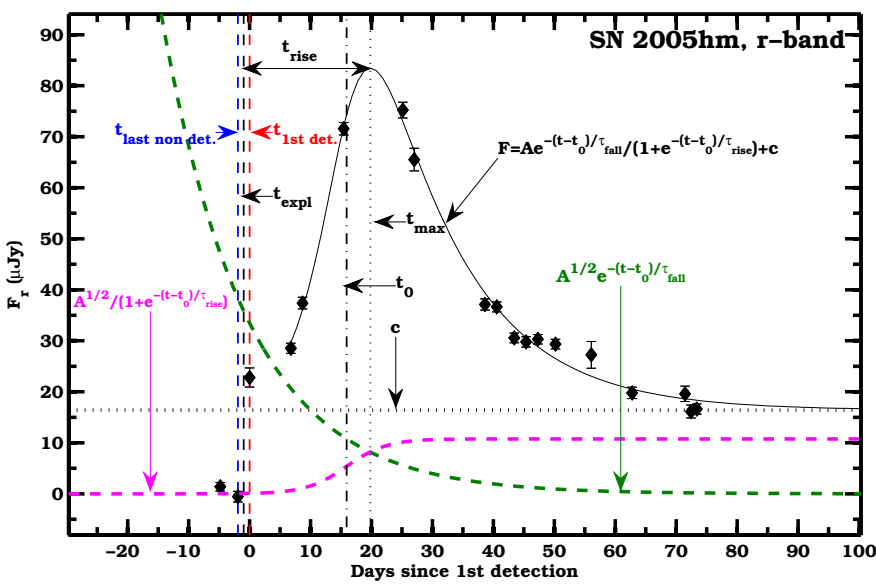

Fig. 8. $r$-band light curve of SN $2005 \mathrm{hm}$ fit by the model given in Eq. (1). We label the different parameters that we discuss in the paper and show the main components of the model that produce a fast rising and exponential declining light curve. The light curve is shown after correction for time dilation.

of Fig. 9, where we plot the time of the peak epoch for each light curve with respect to the epoch of $r_{\max }$ as a function of the effective wavelength $\left(\lambda_{\text {eff }}\right)$ for each filter in the rest frame ${ }^{11}$. The time delay between $u_{\max }$ and $z_{\max }$ is close to five to ten restframe days, depending on the object.

The bottom panel of Fig. 9 displays the peak epochs of each light curve with respect to the peak epoch interpolated to $\lambda_{\mathrm{eff}}\left(r_{z=0}\right)=6231 \AA$ as a function of the effective wavelength of the filter for each object. This comparison reveals a common behavior in the time of the peak in the different bands, which we can describe with a polynomial obtained through a fit to the data. The polynomial coefficients are reported in the caption of Fig. 9. Using this fit, once the epoch of $r_{\max }$ is known for any

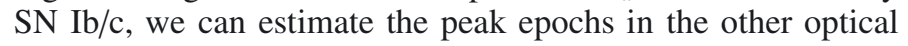
bands with a fairly small uncertainty $(1 \sigma= \pm 1.3$ days). The sequential order of the peak epochs from the bluer to the redder bands reflects the progressive cooling of the photospheric temperature of these objects, as shown in Sect. 3.3.4. This behavior holds for both $\mathrm{SNe} \mathrm{Ib}$ and Ic and has been noticed in several single-object papers (e.g., SN 2004aw, Taubenberger et al. 2006; SN 2007Y, Stritzinger et al. 2009; SN 1999ex Stritzinger et al. 2002; SN 2003jd Valenti et al. 2008; SN 2007gr Hunter et al. 2009; SN 2009bb Pignata et al. 2011; SN 2003dh Lipkin et al. 2004; SN 2010bh Cano et al. 2011), but not previously generalized for a larger SE SN sample (with the exception of the recent paper by Bianco et al. 2014). SNe Ia do not show such a simple relation; for example, Contardo et al. (2000) show that $\mathrm{SNe}$ Ia $I$-band light curves peak before those in the $B$ band.

\subsubsection{Rise times, $t_{\text {rise }}$}

With accurate estimates of the epoch of maximum light for the majority of the $\mathrm{SNe}$, we can establish the rise time in the different bands for those $\mathrm{SNe}$ that have pre-explosion images and therefore a constraint on the explosion epoch $t_{\text {expl }}$. As explained in Sect. 3.1, $t_{\text {expl }}$ is defined as the average between the epochs of last non-detection and first detection, with a conservative uncertainty as half the time interval between them. The difference

\footnotetext{
${ }^{11}$ Effective wavelengths for the SDSS filters are listed at http://skyserver.sdss.org/dr1/en/proj/advanced/color/ sdssfilters.asp
}
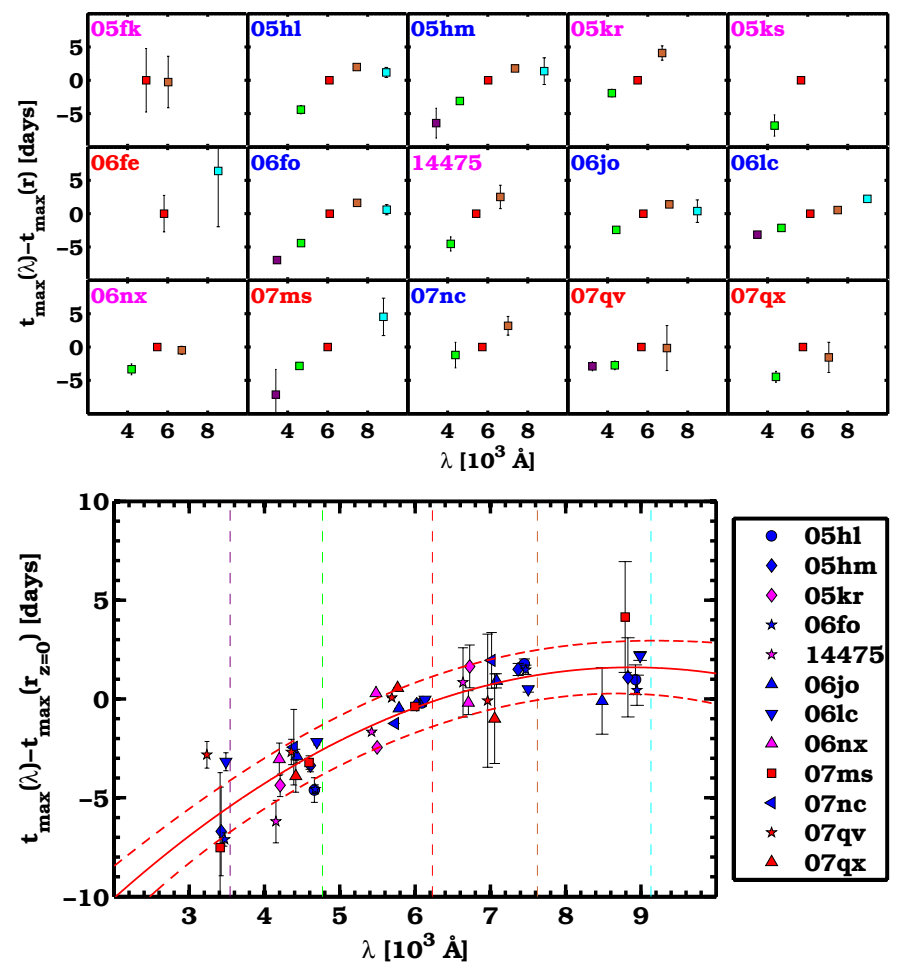

Fig. 9. Top panel: epoch of the peak $\left(t_{\max }\right)$ for each light curve with respect to $t_{\max }$ in the $r$ band as a function of the effective wavelength of the filters in the rest frame for $15 \mathrm{SNe} \mathrm{Ib/c}$ in the SDSS sample (violet, green, red, dark yellow, and cyan represent ugriz filters). Blue, red, and magenta labels correspond to $\mathrm{SNe} \mathrm{Ib}$, Ic, and Ic-BL, respectively. Bottom panel: same as in the top panel, but $t_{\max }$ for each light curve and for each object is plotted with respect to the interpolated $t_{\max }$ at the effective wavelength of $r$ band at redshift $z=0$ (6231 $\AA$ ). Three out of 15 objects (SNe 2005fk, 2005ks, 2006fe) are excluded from the fit since the interpolation to $6231 \AA$ is not possible or reliable (e.g., for SN 2006fe, we do not have the $i$-band maximum epoch). The 2 nd-order polynomial fit to the data (and plotted in red) corresponds to $t_{\max }(\lambda)-t_{\max }\left(r_{z=0}\right)=$ $a \lambda^{2}+b \lambda+c$, where $a=-2.47 \times 10^{-7}, b=+4.38 \times 10^{-3}, c=-17.86 . t_{\max }$ is expressed in days and $\lambda$ in $\AA$. This fit allows predicting peak epochs in the whole optical range given a measured peak in a single band, with little uncertainty ( $\sigma=1.3$ days). Vertical dashed lines indicate the effective wavelengths for the ugriz filters.

between $t_{\text {expl }}$ and the peak epoch $\left(t_{\max }\right)$ corresponds to the rise time $\left(t_{\text {rise }}\right)$; this is reported in the last column of Table 3 for each $\mathrm{SN}$ in each band. The uncertainty on $t_{\text {rise }}$ is the square root of the sum of the squared errors for $t_{\text {expl }}$ and $t_{\max }$, where the uncertainty on the explosion epoch largely dominates. This definition of $t_{\text {expl }}$ ignores a possible faint-plateau phase between core collapse and light curve rise, which has been predicted by the theoretical models of Dessart et al. (2011). We discuss the accuracy and implications of this assumption in Sects. 3.4.1, 3.4.3, and 3.4.4.

In Fig. 10 (top panel), the rise time at the different $\lambda_{\text {eff }}$ for each filter in the SN rest frame is shown (marked by colored squares) for $12 \mathrm{SNe}$. To allow for a direct comparison of the rise times of different objects, we linearly interpolated the observed rise times to the effective wavelengths of ugri filters at redshift $z=0$ (marked by empty diamonds). Given the cadence of the SDSS-II SN survey observations, the uncertainty on the explosion dates, hence on the rise times, is relatively large for most of the objects. However, for six objects we know the $r_{z=0}$-band rise time with an uncertainty $\$ 3$ days. We use these objects, together with other events in the literature with well-constrained 

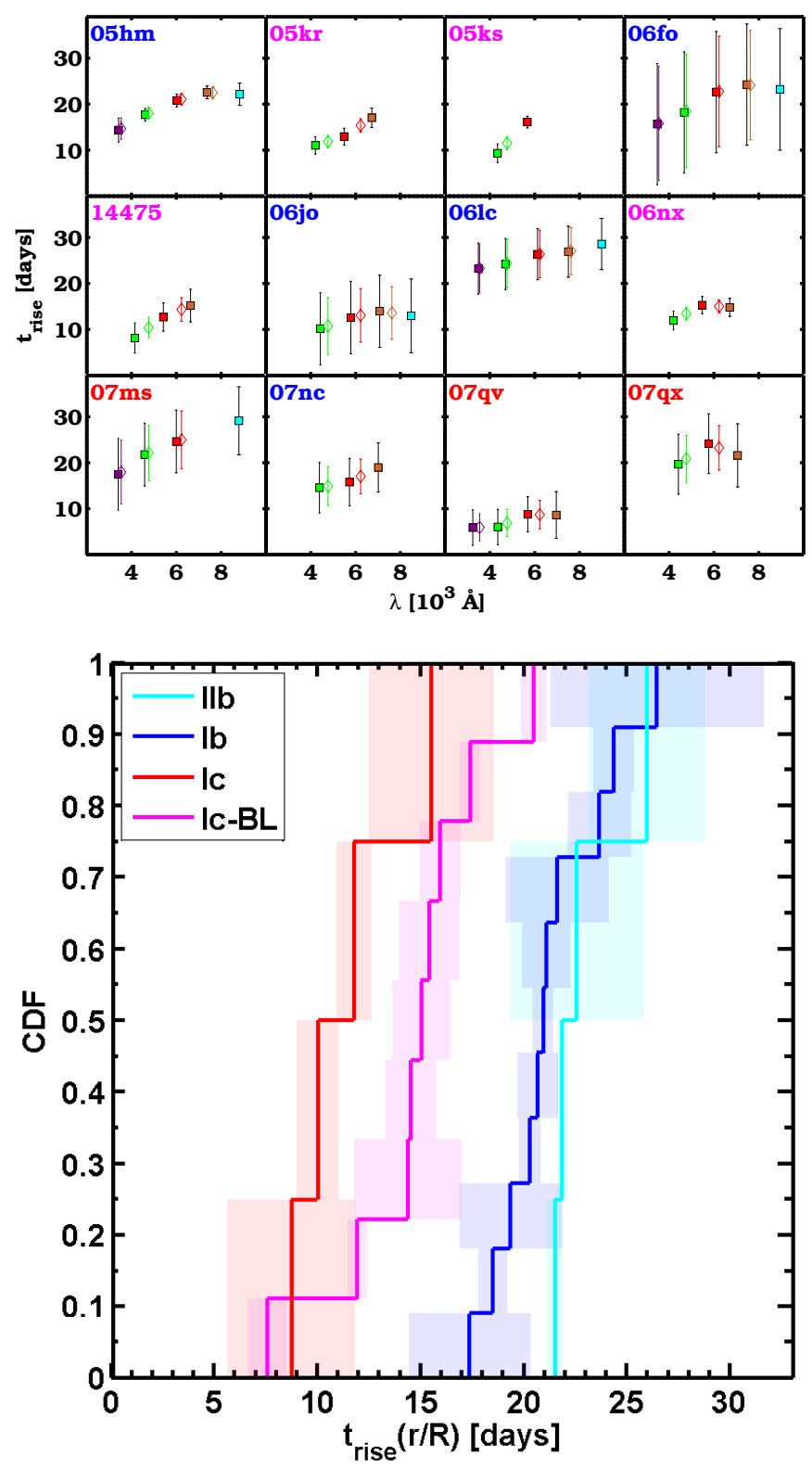

Fig. 10. Top panel: rise times for $12 \mathrm{SNe} \mathrm{Ib} / \mathrm{c}$ in the SDSS sample as a function of the effective wavelength of each filter in the rest frame (violet, green, red, dark yellow, and cyan represent ugriz filters). Bluer bands rise faster than redder bands. Blue, red, and magenta labels correspond to $\mathrm{SNe} \mathrm{Ib}$, Ic, and Ic-BL, respectively. Bottom panel: cumulative distribution functions for the $r / R$-band rise times of $\mathrm{SNe} \mathrm{Ib}(11)$, Ic (4), Ic-BL (9), and IIb (4), from the SDSS sample and from the literature (see Table 4). Helium-poor SNe (Ic, Ic-BL) rise to maximum in a shorter time than helium-rich transients ( $\mathrm{Ib}, \mathrm{IIb}$ ), and this result is statistically significant $(p$-value $<0.05)$. The shaded areas indicate the uncertainties for the rise times.

rise times in the $r / R$-band light curves, to compare the rise times of SNe Ib, Ic, Ic-BL, and also IIb. This comparison is shown in the bottom panel of Fig. 10, where the cumulative distribution function of the rise times is given for the different SN types. (We also indicate the errors on the rise times with shaded area.) All the $r / R$-band rise times are also reported in Table 4 with their errors.

Our result confirms what the data presented in Valenti et al. (2011) suggested: helium-poor SNe (Ic and Ic-BL) rise to maximum faster than helium-rich $\mathrm{SNe}$ (Ib and $\mathrm{IIb}$ ). We found $\left\langle t_{\text {rise }}(I I b, I b, I c, I c-B L)\right\rangle=22.9 \pm 0.8,21.3 \pm 0.4,11.5 \pm 0.5,14.7 \pm$ 0.2 rest frame days, where the quoted uncertainties are the standard deviations in the rise time divided by the square root of the number of objects in each sample $(4,11,4$, and $9 \mathrm{SNe} \mathrm{IIb}, \mathrm{Ib}$, Ic, and Ic-BL, respectively). We note that the sample of SNe Ic is rather small.

The difference between SNe Ib and Ic is statistically significant. A Kolmogorov-Smirnov (K-S) test comparing these two populations gives a $p$-value $=0.001$. Aiming to estimate the robustness of the $p$-value, we made $10^{4}$ Monte Carlo simulations of the rise times ${ }^{12}$ and performed $10^{4} \mathrm{~K}-\mathrm{S}$ tests on the resulting couples of $\mathrm{SN} \mathrm{Ib}$ and $\mathrm{SN}$ Ic rise-time simulations. In this exercise $68 \%$ of the simulated $p$-values were included within 0.005 , confirming the statistical significance of the difference, we measured between these two populations. The bottom panel of Fig. 10 also shows that $\mathrm{SNe} \mathrm{Ib}$ and $\mathrm{SNe}$ Ic-BL display a statistically significant difference. In fact, we found $p$-value $=0.001(0.001)$, where the number in parenthesis corresponds to the value within which $68 \%$ of the simulated $p$ values are included. Statistically significant differences were also found when we compared SNe IIb to Ic, $p$-value $=0.011(0.011)$, and $\mathrm{SNe}$ IIb to Ic-BL, $p$-value $=0.002(0.009)$. The peculiar SN Ic 2011 bm (Valenti et al. 2012) was excluded from the rise time comparison, which has an unusual $\sim 40$ days rise time.

\subsection{3. $\Delta m_{15}$ and $\Delta m_{-10}$}

Besides the rise time, another important parameter that characterizes the light curve shapes of radioactively powered SNe is $\Delta m_{15}$, i.e., the difference in magnitude between the value at peak and at 15 days (in the rest frame) past maximum $\left[\Delta m_{15}=-2.5 \log _{10}\left(F\left(t_{\max }+15\right) / F_{\max }\right)\right]$. It is particularly useful for $\mathrm{SNe} \mathrm{Ia}$, where its correlation to peak brightness allows for their use as standardizable candles (Phillips 1993). GRB SNe (Li \& Hjorth 2014; Cano 2014) have also been recently suggested as standardizable candles showing luminosity-decline relations ( $\mathrm{Li}$ et al. 2014; Cano \& Jakobsson 2014). From the fits on the light curves we derived $\Delta m_{15}$ for $15 \mathrm{SNe}$ in the sample, and plot the measured values as a function of the effective wavelength of each filter in Fig. 11 (top panel). It is clear that $\Delta m_{15}$ is larger for the bluer bands than for the redder ones. A similar trend is observed in the optical light curves of SNe Ia (Folatelli et al. 2010). When we overplot all the $\Delta m_{15}$ values as a function of wavelength in the central panel of Fig. 11, the common $\Delta m_{15}$ behavior for all the SDSS-II SNe Ib/c becomes evident. If we fit a polynomial to these data, an analytical expression for $\Delta m_{15}$ as a function of the effective wavelength of the filters can be derived (Fig. 11, see the caption for the polynomial expression).

Because we have all the $\Delta m_{15}$ values in different filters, we linearly interpolated the observed $\Delta m_{15}$ at the effective wavelength of the gri filters at $z=0$, to allow for a direct comparison between the different $\mathrm{SN}$ types. In the bottom panel of Fig. 11, we show that the $\Delta m_{15}$ distribution for the $r_{z=0}$ band of SNe Ib does not differ significantly from that of SNe Ic or Ic-BL. Here we consider the SDSS-II data and the $\Delta m_{15}(R)$ from Table 5 of Drout et al. (2011), where a couple of SNe IIb are also reported. We included a total of 2, 14, 14, and $9 \mathrm{SNe} \mathrm{IIb}$, Ib, Ic, and Ic-BL, respectively. The $\Delta m_{15}$ distributions for $\mathrm{SNe} \mathrm{Ib}$, Ic, and Ic-BL are also similar when we consider the $g_{z=0}$ instead of the $r_{z=0}$ band (including only the SDSS-II data).

12 For each event we produced $10^{4}$ normally distributed random rise times with central values of the normal distribution equal to the measured rise time of the $\mathrm{SN}$ and $\sigma$ equal to the rise-time uncertainty. 

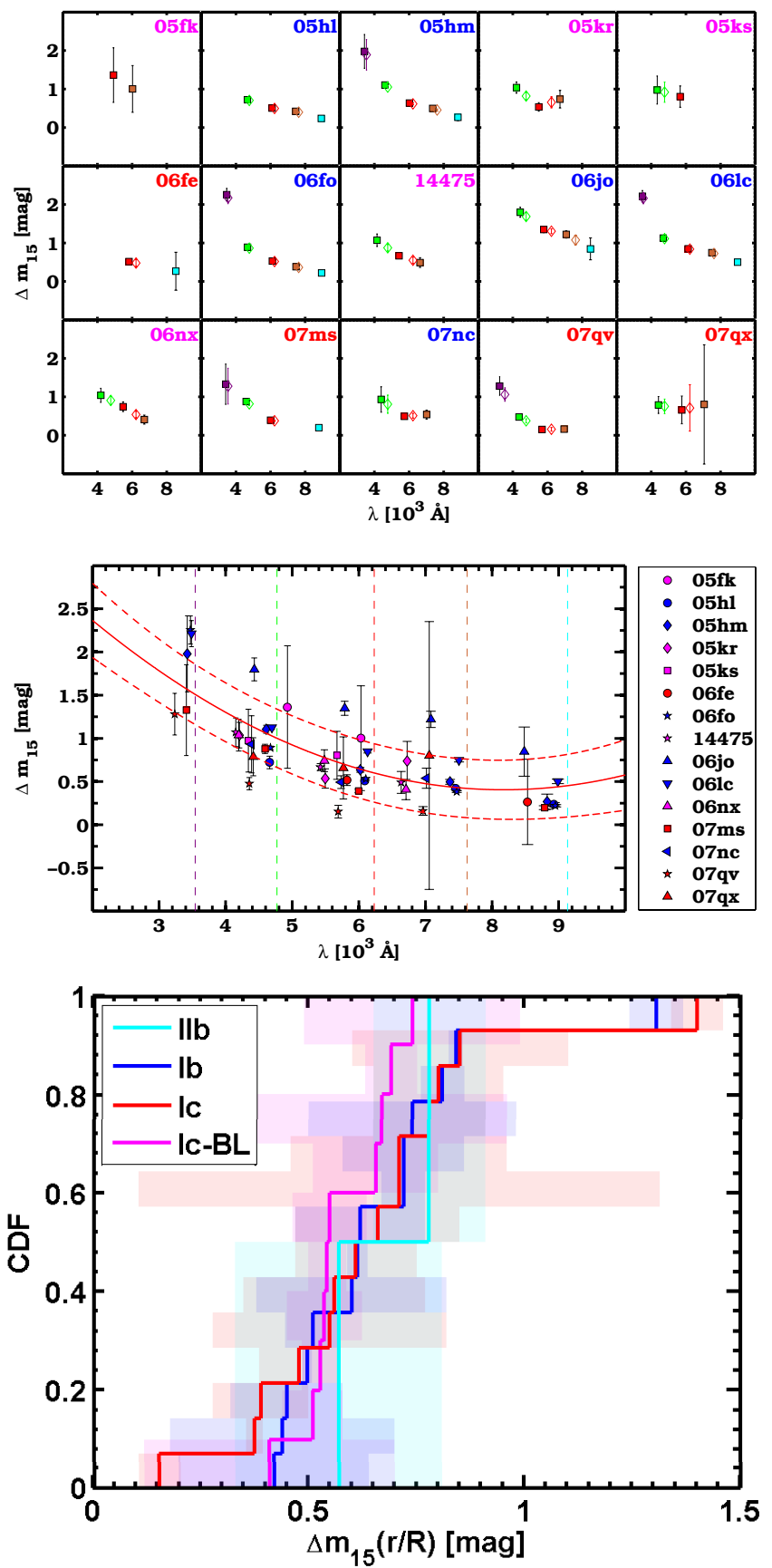

Fig. 11. Top panel: $\Delta m_{15}$ for $15 \mathrm{SNe} \mathrm{Ib} / \mathrm{c}$ in the SDSS sample as a function of the effective wavelength of each filter in the SN rest frame (violet, green, red, dark yellow and cyan represent ugriz filters). Bluer bands decline faster than redder bands. Central panel: Same as in the top panel, the 2 nd order polynomial fitted to the data (and plotted in red) corresponds to $\Delta m_{15}(\lambda)=a \lambda^{2}+b \lambda+c$, where $a=5.14 \times 10^{-8}$, $b=-8.41 \times 10^{-4}$ and $c=3.84 . \Delta m_{15}$ is expressed in magnitudes and $\lambda$ in $\AA$. Typical $1 \sigma$ uncertainties on $\Delta m_{15}(\lambda)$ are on the order of 0.4 mag. Vertical dashed lines indicate the effective wavelengths for the ugriz filters. Bottom panel: cumulative distribution functions for the $r / R$-band $\Delta m_{15}$ of SNe Ib (14), Ic (14), Ic-BL (10) and IIb (2), from the SDSS sample and from Drout et al. (2011). SDSS $\Delta m_{15}$ values are those interpolated at $\lambda_{\mathrm{eff}}\left(r_{z=0}\right)$. Shaded areas indicate the uncertainties for $\Delta m_{15}$. There is no significant difference among the distributions of the different classes, with $p$-values $>0.05$.

The width of the light curves on the rising part is evaluated by measuring $\Delta m_{-10}$, i.e., the difference in magnitude between the value at peak and what was measured ten days before
$\operatorname{maximum}\left[\Delta m_{-10}=-2.5 \log _{10}\left(F\left(t_{\max }-10\right) / F_{\max }\right)\right]$. This provides an estimate for the steepness of the rise, which is independent of knowledge of the exact explosion date, and the potential presence of an early plateau phase. In Fig. 12 (top panel) we show the rest frame $\Delta m_{-10}$ values for each $\mathrm{SN}$ in the sample as a function of the effective wavelength of the filters (colored squares). We also interpolate (empty symbols) these values at the gri effective wavelengths for $z=0$ in order to obtain $\Delta m_{-10}$ values suitable for a comparison among the different $\mathrm{SN}$ types.

The $\Delta m_{-10}$ values are higher at short wavelengths than in the redder bands; i.e., the SNe rise is steeper in the bluer bands. This is confirmed by overplotting the data for all $14 \mathrm{SNe}$ in the central panel of Fig. 12. A linear fit to the data is shown and its expression is reported in the corresponding caption. The scatter around the fit is $\sim 0.66 \mathrm{mag}$. We compare the $\Delta m_{-10}$ values interpolated at $r$-band effective wavelengths among the different SN types (Fig. 12, bottom panel). Here we consider the $\Delta m_{-10}$ values of our $\mathrm{SNe}$ (excluding those with extrapolated values of $\Delta m_{-10}$ ) and those of other $\mathrm{SNe} \mathrm{Ib} / \mathrm{c}$ (and IIb) whose $r / R$-band light curves are available in the literature (see Table 4). SNe Ic and SNe Ic-BL exhibit a steeper rise compared to SNe Ib and IIb, consistently with the different rise times. The difference between $\mathrm{SNe} \mathrm{Ib}$ and Ic is marginally significant ( $p$-value $=0.18$ ), whereas the K-S test between $\mathrm{SNe} \mathrm{Ib}$ and Ic-BL gives $p$-value $=0.003$.

Armed with the interpolated values of $\Delta m_{-10}$ and $\Delta m_{15}$ at the effective wavelength of the gri filters, we searched for a correlation between these two parameters. Apart from the single object SN 2006jo, which displays high values for both parameters in all bands, there is little evidence of any strong correlation between the shape of the rising part and the falling part of the light curve. The rise time of SNe Ia is also not strongly correlated with the fall, even though the slowest decliners tend to be among the fastest risers (Hayden et al. 2010a).

\subsubsection{Light curve templates}

The previous analysis showed that the light curve shapes for $\mathrm{SNe} \mathrm{Ib}$ and Ic/Ic-BL are similar overall. Using the light curve fit and the derived parameters for most of the objects, we construct $\mathrm{SN} \mathrm{Ib/c} \mathrm{light} \mathrm{curve} \mathrm{templates} \mathrm{in} \mathrm{the} \mathrm{ugriz} \mathrm{bands.} \mathrm{We} \mathrm{selected}$ only objects at redshift $z<0.1$ in order to avoid the need for large $k$-corrections. Each light curve was normalized by the flux at the peak, and the time axis was shifted so that the epoch of the peak is at zero. Following the approach by Hayden et al. (2010a) for SNe Ia, we applied a double stretch to the ugriz-band light curves. We measured the half width at half maximum (HWHM) before and after the peak for each light curve and took the ratio between each HWHM and its average value in the sample as the stretch factor, both before and after the peak. The result for the ugriz bands is shown in Fig. 13. A single stretch based on the full width at half maximum (FWHM) of the light curves would lead to a higher dispersion on the rising part of the normalized light curves, which is obviously reduced by applying a double-stretch correction. For the $r$ band, with a double stretch, we obtain a fit with RMS $=0.046$ (in normalized flux units), whereas the RMS is 0.052 (in normalized flux units) when a single stretch is applied. We fit the double-stretched and normalized light curves with polynomials to obtain templates, which are made available online ${ }^{13}$. It is confirmed that the bluer bands rise and decline faster than the redder bands. The best fit is shown in Fig. 13. At

\footnotetext{
${ }^{13}$ http://ttt.astro.su.se/ ftadd/template.txt and at the CDS.
} 

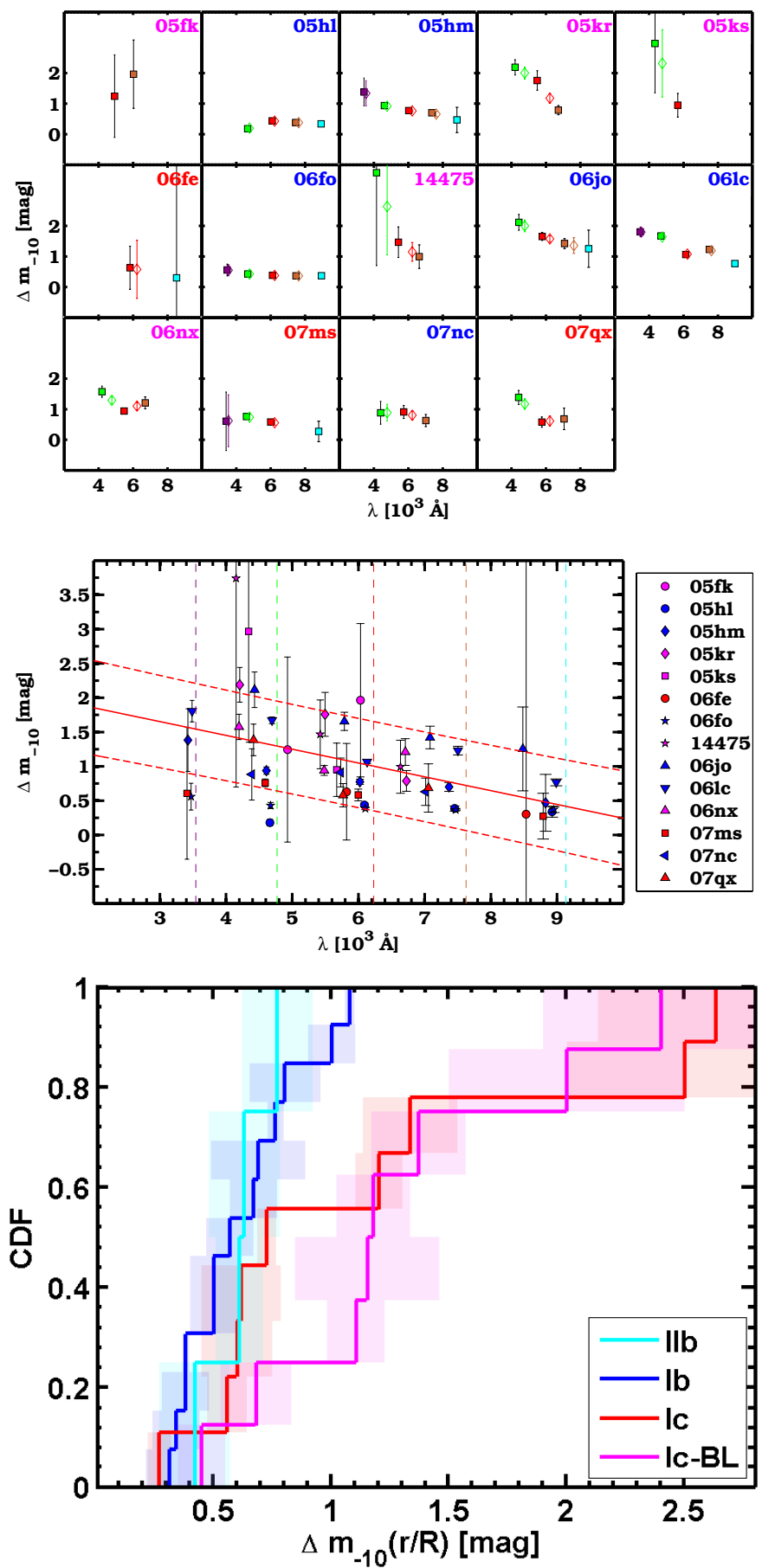

Fig. 12. Top panel: $\Delta m_{-10}$ for $14 \mathrm{SNe} \mathrm{Ib} / \mathrm{c}$ in the SDSS sample as a function of the effective wavelength of each filter in the SN rest frame (violet, green, red, dark yellow and cyan represent ugriz filters). Bluer bands rise faster than redder bands for most of the objects. Blue, red and magenta labels correspond to $\mathrm{SNe} \mathrm{Ib}$, Ic and Ic-BL, respectively. Central panel: same as in the top panel, the line fitted to the data (and plotted in red) corresponds to $\Delta m_{-10}(\lambda)=-2.01 \times 10^{-4} \lambda+2.26$, with $\Delta m_{-10}$ expressed in magnitudes and $\lambda$ in $\AA$. Uncertainties on $\Delta m_{-10}(\lambda)$ are $\sim 0.66$ mag. Bottom panel: cumulative distribution functions for the $r$-band $\Delta m_{-10}$ of SNe IIb (4), Ib (13), Ic (9), and Ic-BL (8) from the SDSS sample and from the literature. Shaded areas indicate the uncertainties for $\Delta m_{-10}$. SNe Ic and Ic-BL show higher values of $\Delta m_{-10}$ when compared to $\mathrm{SNe} \mathrm{Ib}$ ( $p$-values $=0.18$ and 0.003 , respectively).

early times, only a few points are clearly off with respect to the best fit and were excluded (in the ugr bands). These points belong to SNe 2005hm and 2007qx and are likely to be signatures
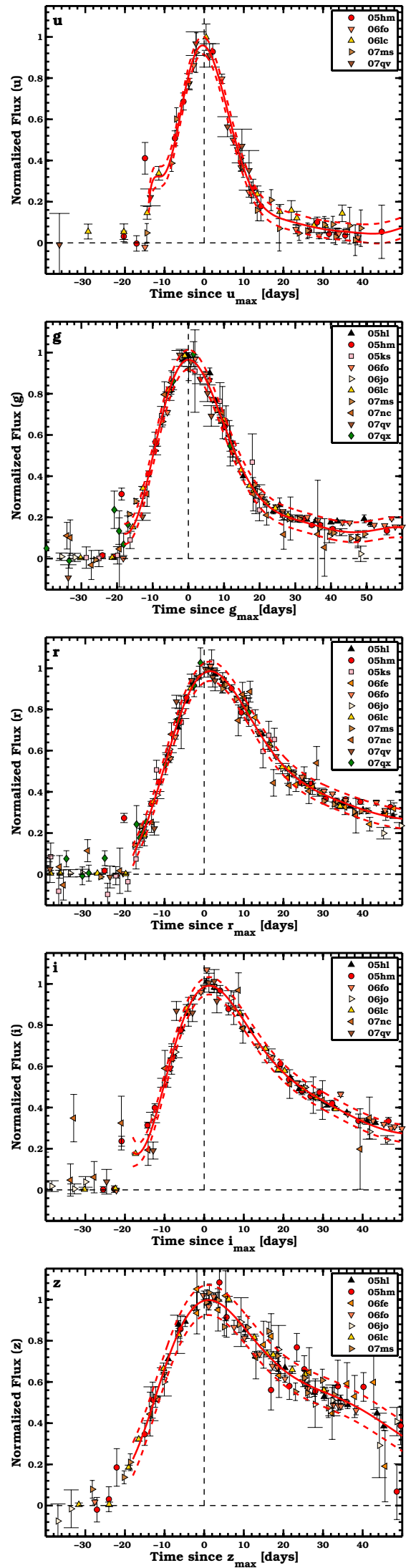

Fig. 13. Normalized and double-stretched ugriz light curves of $\mathrm{SNe} \mathrm{Ib} / \mathrm{c}$ from the SDSS sample. Only the objects at redshift $z<0.1$ were selected. We show each object individually and fit the data with a polynomial (12th order). The best fit is shown by a red solid line, uncertainties by dashed red lines. We make these templates available in electronic form at the CDS. 

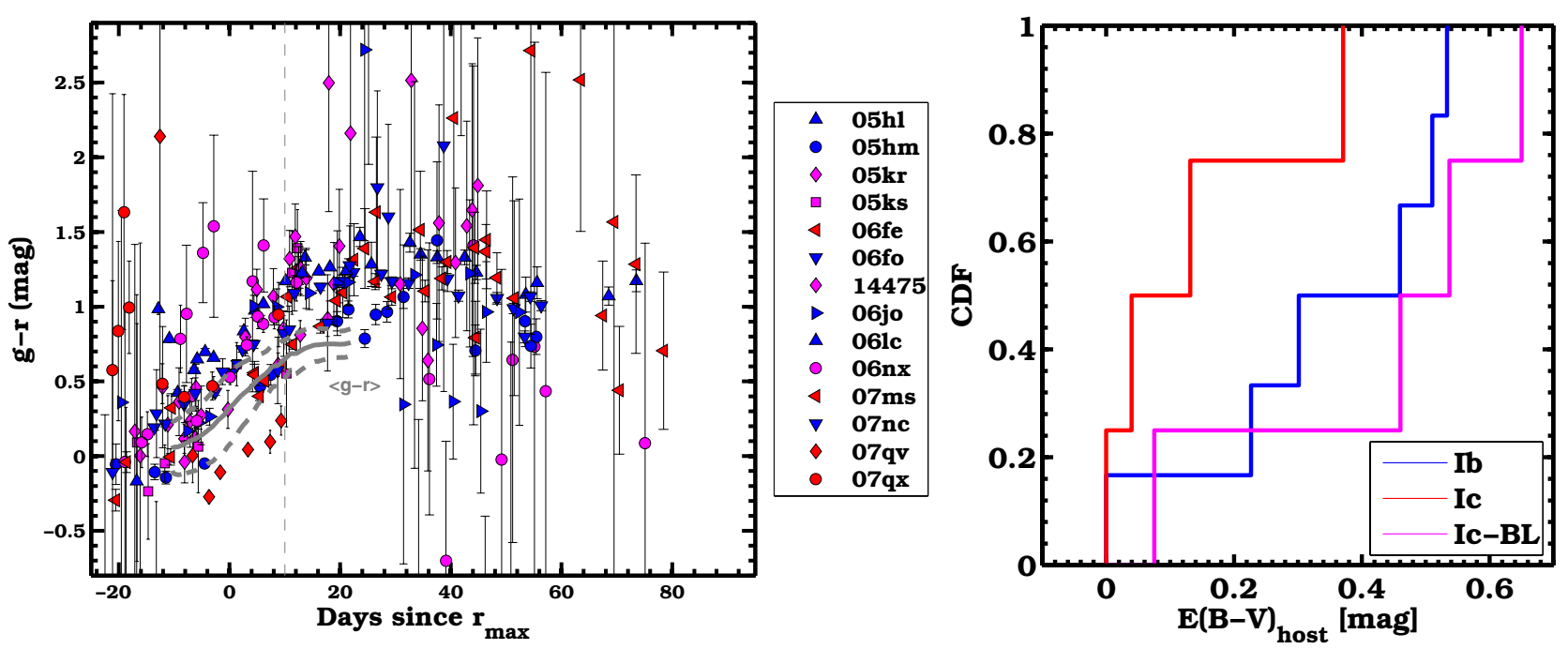

Fig. 14. Lefthand panel: $g-r$ color (corrected only for the Galactic extinction) as a function of time since $r_{\max }$ for 14 SDSS SNe Ib/c. The average extinction-corrected $\langle g-r\rangle$ color of seven SE SNe (see Sect. 3.3.1 for details) is shown by a solid gray line. Its value at 10 days (this epoch is labeled by a vertical dashed gray line, where $g-r=0.64 \pm 0.13 \mathrm{mag}$ ) is assumed as the intrinsic colour for computing the host extinction for each SN. A Cardelli et al. (1989) reddening law with $R_{V}=3.1$ is adopted in the computation. Righthand panel: cumulative distribution functions of $E(B-V)_{\text {host }}$ for SNe Ib, Ic, and Ic-BL. The corrections for SNe Ib and Ic-BL appear larger than those needed for SNe Ic. The uncertainty on each $E(B-V)_{\text {host }}$ is $0.2 \mathrm{mag}$.

of the shock break-out cooling tail observed in these events (see Sect. 3.3.5).

\subsubsection{Summary of the light curve properties}

We list some main results that we determined through the analysis described in the previous sections:

- Both SNe Ib and SNe Ic/Ic-BL peak first in the bluer and then successively in the redder bands.

- $\mathrm{SNe}$ Ic and Ic-BL rise to maximum in a shorter time than $\mathrm{SNe} \mathrm{Ib}$ and IIb, a result that is statistically significant in the $r$ band.

- The steepness of the light curves after maximum $\left(\Delta m_{15}\right)$ is similar for the different SE SN types.

- The steepness of the light curves on the rise $\left(\Delta m_{-10}\right)$ is greater for $\mathrm{SNe}$ Ic-BL and Ic than for $\mathrm{SNe} \mathrm{Ib}$ and IIb.

- $\Delta m_{15}$ and $\Delta m_{-10}$ are larger in the bluer bands.

\subsection{Light-curve absolute maxima and bolometric properties}

\subsubsection{Host extinction estimates}

Lacking high resolution spectra for our objects, we cannot use the relations between extinction and $\mathrm{NaI} \mathrm{D}$ equivalent width (EW) suggested in the literature (e.g., Poznanski et al. 2012). Our low resolution spectra are not of sufficient quality to measure the EW of the unresolved Na I D lines (which is anyway a poor proxy of the extinction, Poznanski et al. 2011). Therefore, following the approach by Drout et al. (2011), we based our host extinction estimates on SN colors. In particular we make use of $g-r$ as this color is the closest to $V-R$, which is the one used by Drout et al. (2011). Also, $g$ - and $r$-band light curves show higher quality compared to those obtained with other filters in our dataset.

First we determine the average, galaxy and host extinctioncorrected $\langle g-r\rangle$ from seven SE SNe in the literature with wellknown host extinction, available $g$-band and $r$-band photometry, and well-known $r$-band peak epoch. We stress that, as in
Drout et al. (2011), the host extinction value reported in the literature for each object is in itself uncertain, given that most of them are obtained through the EW of unresolved $\mathrm{Na}$ I D lines. We select objects belonging to the different SE-SN subclasses: SNe Ib 2007Y (Stritzinger et al. 2009), 2008D (Soderberg et al. 2008), 2009jf (Valenti et al. 2011), SNe Ic 2009bb (Pignata et al. 2011), 20011bm (Valenti et al. 2012), and SNe IIb 2010as (Folatelli et al. 2014), 2008ax (Pastorello et al. 2008). The $\langle g-r\rangle$ evolution along with its standard deviation are shown by grey lines in Fig. 14 (lefthand panel). The dispersion of $\langle g-r\rangle$ has its minimum $(0.13 \mathrm{mag})$ about ten days past $r_{\max }$, similar to what Drout et al. (2011) show for their $\langle V-R\rangle$. Therefore we chose the value of $\langle g-r\rangle$ at this epoch $\left(\langle g-r\rangle_{10}=0.64 \pm 0.13 \mathrm{mag}\right)$ as the intrinsic color.

We then interpolate at ten days past $r_{\max }$ all the $g-r$ profiles of our SDSS SNe (corrected only for the Galactic extinction), which are shown in Fig. 14 (lefthand panel). The difference in color $(E(g-r))$ at this phase between each $\mathrm{SN}$ and $\langle g-r\rangle$ is assumed to only be produced by the effect of host extinction. We assume a Cardelli et al. (1989) reddening law with $R_{V}=3.1$ to obtain $E(B-V)_{\text {host }}$ from $E(g-r)$. The derived values for $E(B-V)_{\text {host }}$ are reported in Table 1 . The uncertainty on the derived $E(B-V)_{\text {host }}$ is $0.2 \mathrm{mag}$, as derived from the dispersion of $\langle g-r\rangle$. SNe Ic exhibit smaller host-extinction estimates compared to those of SNe Ib and Ic-BL ( $p$-value $=0.25$ and 0.11$)$, as shown in Fig. 14. Two events are found to be unreddened (SNe 2005hm and 2007qv; the latter is found to be intrinsically bluer than typical SE SNe).

In the following, we do not simply add the Galactic and the host-galaxy color excess to compute the extinction in each filter. Instead, we compute the rest-frame extinction separately, because it depends on the redshift. This is important for the most distant objects of our sample.

\subsubsection{Light curve absolute maxima, $L_{v}^{\max }$}

The fit of the light curves presented in Sect. 3.1 also provides the value of the observed peak fluxes (see $F_{\max }$ in Table 3 ). 

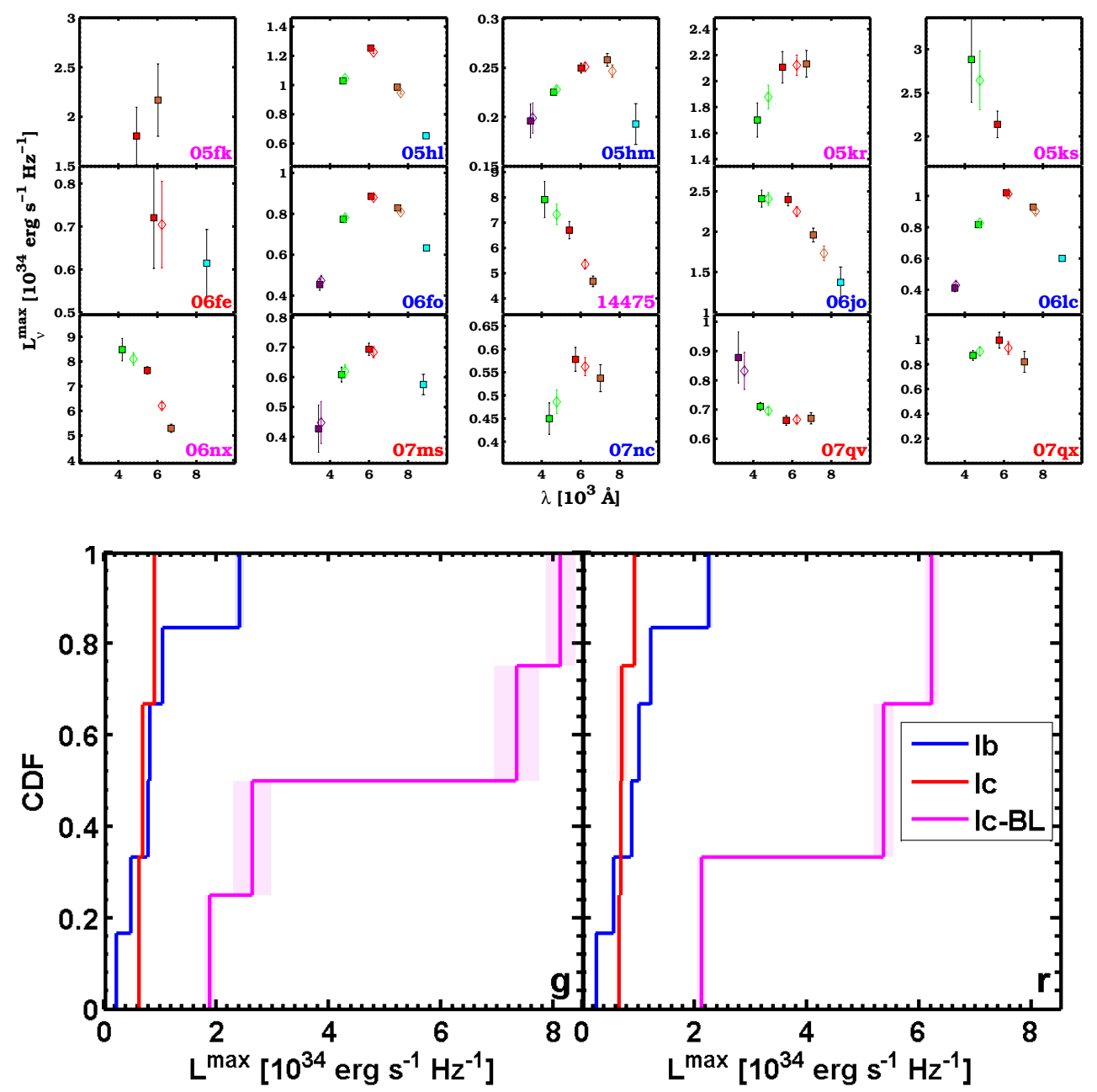

Fig. 15. Top panel: peak luminosity per unit frequency as a function of the effective wavelength of the filter in the rest frame for $15 \mathrm{SNe} \mathrm{Ib} / \mathrm{c}$ in the SDSS sample (with host-extinction corrections). Violet, green, red, dark yellow, and cyan correspond to ugriz filters. Blue, red, and magenta labels correspond to SNe Ib, Ic and Ic-BL, respectively. Bottom panels: cumulative distributions of the peak luminosity per unit frequency in $g_{z=0}($ left $)$ and $r_{z=0}$ (right) bands for SNe Ib (6), Ic (3-4) and Ic-BL (4-3) in the SDSS sample. Shaded areas indicate the uncertainties for $F_{\max }$. SNe Ic-BL are clearly brighter than $\mathrm{SNe} \mathrm{Ib}$ and Ic.

With the known luminosity distance $\left(D_{L}\right)$ and total extinction for each object (see Table 1), we compute the peak luminosities (per unit frequency, $L_{\gamma}^{\max }$ ) for each light curve and $\mathrm{SN}$ $\left(L_{v_{e}}^{\max }=4 \pi D_{L}^{2}(1+z)^{-1} F_{v_{\mathrm{o}}}^{\max }\right)$. Here $v_{\mathrm{o}}$ corresponds to the observed effective frequency of the SDSS filter, while $v_{e}$ corresponds to the rest frame of the SN. To compare the results among objects with different redshifts, we interpolate the $L_{v}^{\max }$ at the effective wavelengths of the gri filters. $L_{v}^{\max }$ as a function of wavelength is shown in the top panel of Fig. 15, where the empty diamonds represent the interpolated fluxes. The cumulative distributions of $L_{v}^{\max }$ in the $g$ and $r$ bands are shown for SNe Ib, Ic and Ic-BL in the bottom panel of Fig. 15. These plots show that SNe Ic-BL are clearly more luminous than $\mathrm{SNe} \mathrm{Ib}$ and Ic in both bands, with high significance ( $p$-value $\leq 0.05$ in both bands). SNe Ic and Ib have a similar distribution of $L_{v}^{\max }$. This result is similar to what was found by Cano (2013).

If we exclude the host-extinction corrections, SNe Ic-BL are still more luminous than both $\mathrm{SNe}$ Ic and $\mathrm{SNe} \mathrm{Ib}$, and $\mathrm{SNe}$ Ic appears slightly brighter than $\mathrm{SNe} \mathrm{Ib}$ ( $p$-value $=0.20,0.13$ for the $g$ and $r$ bands, respectively). A similar trend was visible for the $V$ and $R$ bands in the sample by Drout et al. (2011, their Fig. 10).

\subsubsection{Pseudo-bolometric luminosity}

Using $D_{L}$, multi-band fluxes, $E(B-V)_{\mathrm{MW}}$ and $E(B-V)_{\text {host }}$, we computed pseudo-bolometric ugriz light curves for each SN. Each broadband flux was corrected for the extinction computed at its corresponding wavelength assuming a Cardelli et al. (1989) reddening law $\left(R_{V}=3.1\right)$. For each epoch, the ugriz SED was de-redshifted, multiplied by $1+z$, and integrated between rest frame $3500 \AA$ and $9000 \AA$, in order to compare the same SED portion for each event. The resulting flux was then multiplied by $4 \pi D_{L}^{2}$ to obtain the pseudo-bolometric luminosity $(L)$, and is displayed in Fig. 16. Each pseudo-bolometric light curve observed before and after maximum was fit with the expression in Eq. (1), and the resulting best fit is shown. This fit allowed us to measure the peak luminosity and its epoch, as well as the parameters derived for the single-band light curves. In Table 5 we report these parameters for 14 pseudo-bolometric light curves.

Comparing the host extinction-corrected $L_{\max }$ between $\mathrm{SNe} \mathrm{Ib}$ and Ic, we found that the two distributions overlap (Fig. 17, lefthand panel). SNe Ic-BL are more luminous than the other types, with high statistical significance $(p<0.05)$. This result was also found by Drout et al. (2011) and Cano (2013). If the host-extinction corrections are neglected, SNe Ic appear 
F. Taddia et al.: Early-time light curves of SDSS-II SNe Ib/c

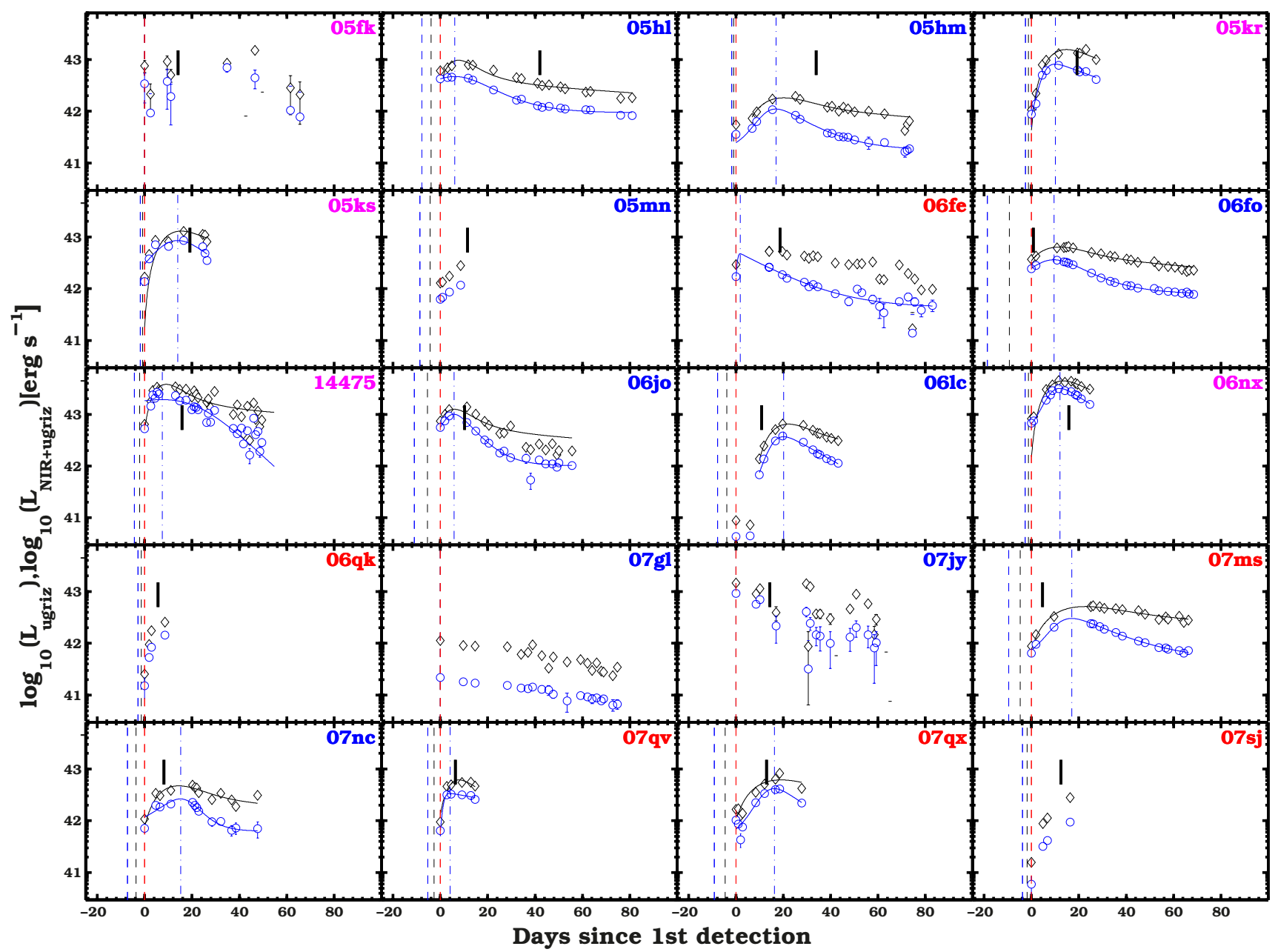

Fig. 16. Pseudo-bolometric ( $L_{\text {ugriz }}$, blue circles) and bolometric ( $L_{\mathrm{NIR}+u g r i z}$, black diamonds) light curves for the sample of SNe Ib/c from SDSS. Discovery epochs are marked by red dashed lines, the last non-detections by dashed blue lines. Each pseudo-bolometric light curve has been fit with Eq. (1), and the best fit is shown by a solid blue line. The peak epoch is marked by a blue/black dashed-dotted line. Each bolometric light curve has been fit with the Arnett model (see Sect. 3.4.2) when the explosion date and the maximum epoch are known, and the best fit is shown by a black solid line. The epoch of the first spectrum is marked by a black segment. Blue, red, and magenta labels correspond to SNe Ib, Ic, and Ic-BL, respectively.
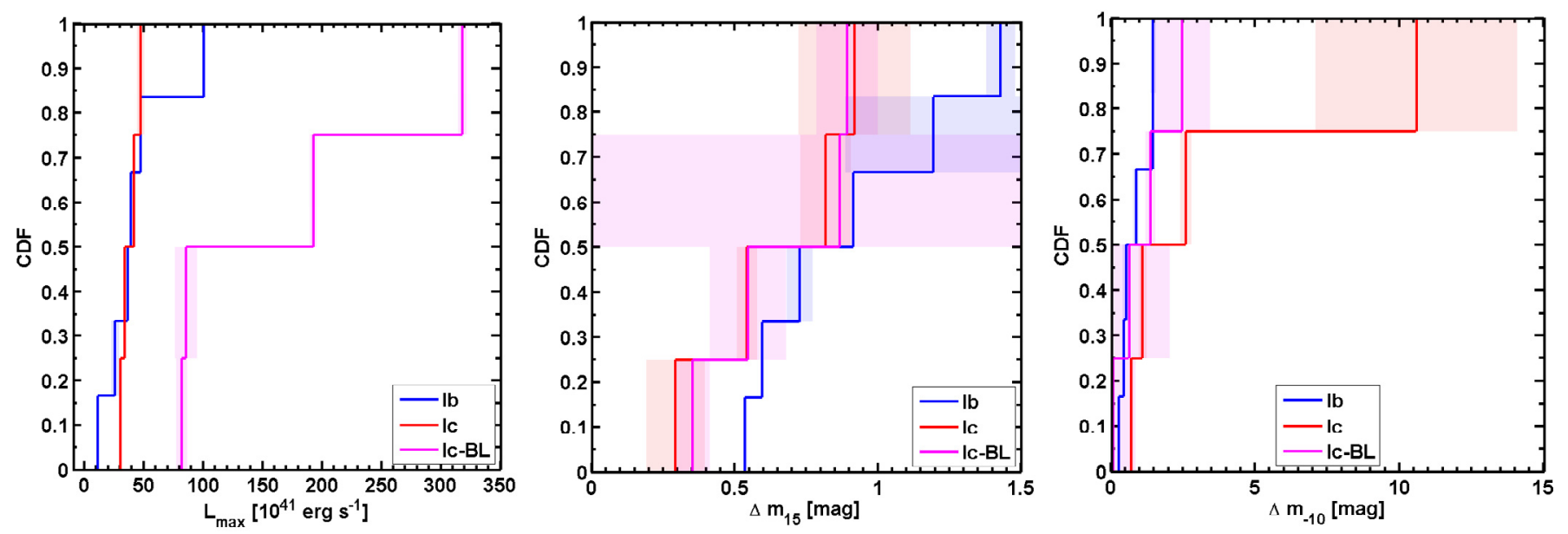

Fig. 17. Lefthand panel: cumulative distributions for the pseudo-bolometric peak luminosity with host-extinction correction. SNe Ib and Ic show similar distributions, SNe Ic BL are more luminous. Center panel: $\Delta m_{15}$ of SNe Ib, Ic and Ic-BL. There is no significant difference among the three SN classes. Righthand panel: $\Delta m_{-10}$ of SNe Ib, Ic and Ic-BL. Helium-poor SNe show higher values compared to those of SNe Ib. Shaded areas indicate the uncertainties for each $\mathrm{SN}$. 


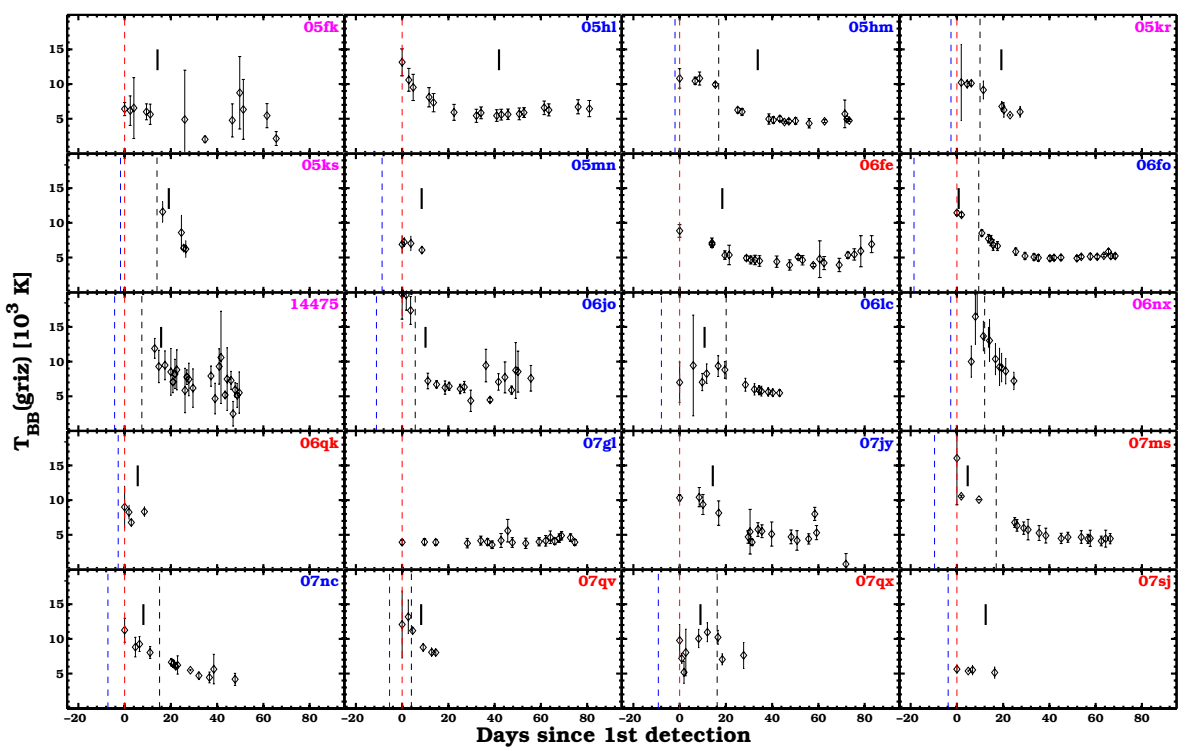

Fig. 18. Temperature evolution for the 20 SDSS $\mathrm{SNe} \mathrm{Ib} / \mathrm{c}$ from the $\mathrm{BB}$ fits on the griz SEDs. Discovery epochs are marked by red dashed lines, the last non-detections by dashed blue lines. The epoch of the first spectrum is marked by a black segment. Here we include the hostextinction corrections. Maximum luminosity epochs are marked by black, vertical dashed lines. Blue, red, and magenta labels correspond to $\mathrm{SNe} \mathrm{Ib}$, Ic, and Ic-BL, respectively.
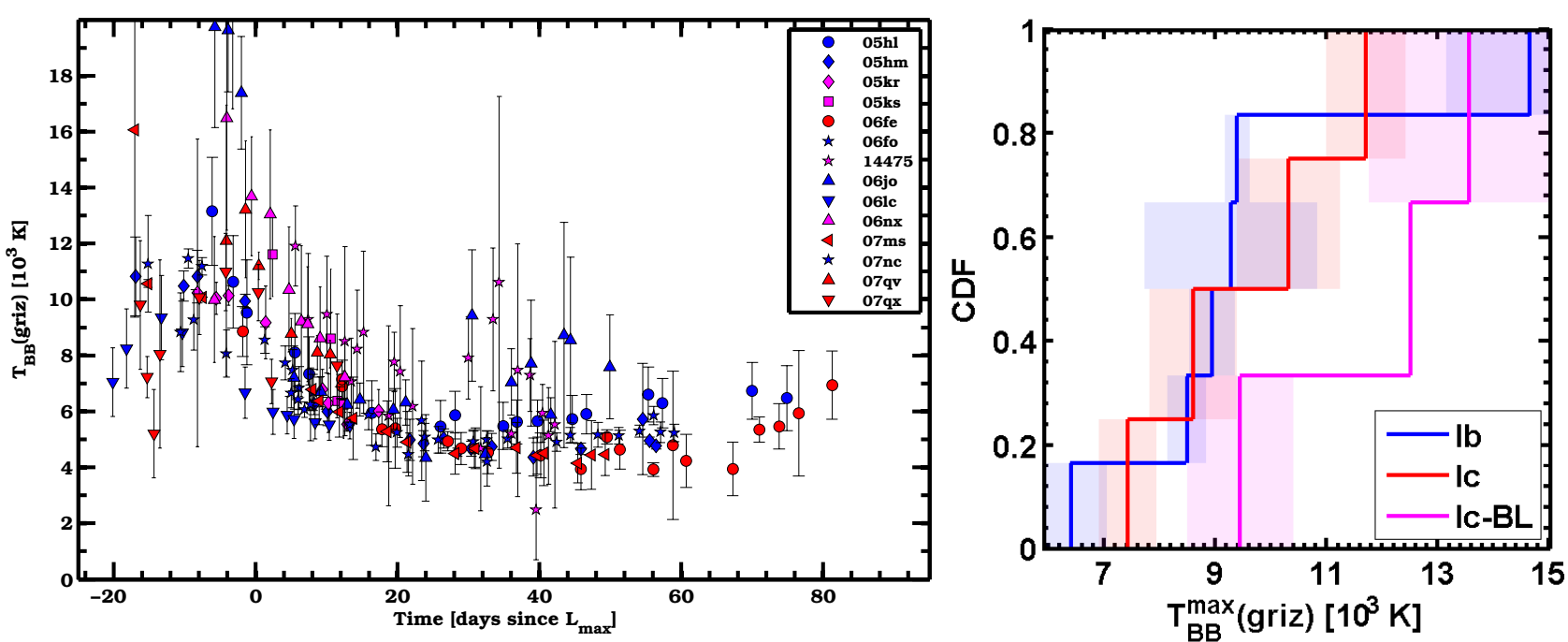

Fig. 19. Lefthand panel: temperature evolution for the 14 SDSS SNe Ib/c with known $L_{\max }$ epoch. Here we include host-extinction corrections. The temperature evolution is similar for all the events. Righthand panel: cumulative distributions for the temperatures at maximum light of SNe Ib, Ic, and Ic-BL. Here we include host-extinction corrections. There is no significant difference among the three SN types. Shaded areas indicate the uncertainty for each SN.

brighter than $\mathrm{SNe} \mathrm{Ib}$, consistent with the individual filtered peak fluxes but with higher statistical significance ( $p$-value $=0.07)$.

There is no visible difference between the SN types when we compare the pseudo-bolometric $\Delta m_{15}$ (Fig. 17, bottom left panel). The pseudo-bolometric $\Delta m_{-10}$ comparison (bottom right panel of Fig. 17) reveals that SNe Ic and Ic-BL have slightly higher values than those of $\mathrm{SNe} \mathrm{Ib}$ (same trend as in Fig. 12, this time with $p$-value $=0.43$, respectively). These results do not strongly depend on the extinction corrections.

\subsubsection{Temperature}

To compute the temperature $\left(T_{\mathrm{BB}}\right)$ evolution for each object, we fitted the rest-frame SEDs (including host-extinction correction) with a black-body (BB) function. We excluded the $u$ band from the fit, given the strong line-blanketing effect at these wavelengths (also $g$ band is partially affected), as well as the large statistical errors (due to poor sensitivity) for this pass-band for many $\mathrm{SNe}$, as well as large extinction uncertainties.

We show the $T_{\mathrm{BB}}$ evolution in Fig. 18 for each object. It is clear that $T_{\mathrm{BB}}$ generally decreases in the first $\sim 30$ days, until it reaches an almost constant value. For a few objects (e.g., SNe 2006lc and 2007qx) we also observe a smooth rise in the first days after detection, since we likely detected them soon after outburst. In Fig. 19 (lefthand panel) we plot all the $T_{\mathrm{BB}}$ profiles to highlight their similar evolution. Around maximum light, the measured temperatures after host-extinction corrections are similar among the three $\mathrm{SN}$ classes $\left(T_{\mathrm{BB}}^{\max }=7000-15000 \mathrm{~K}\right.$, only SNe Ic-BL appear marginally hotter). This is a consequence of the assumption that all the SNe have the same intrinsic value of $g-r$ at just ten days past maximum. Figure 19 (righthand panel) displays the cumulative distribution functions (CDFs) of $T_{\mathrm{BB}}^{\max }$ for $\mathrm{SNe} \mathrm{Ib}$ and $\mathrm{Ic} / \mathrm{Ic}-\mathrm{BL}$. The temperatures at tens of days after maximum light are less affected by the extinction corrections given that most of the flux at those epochs is emitted in the 


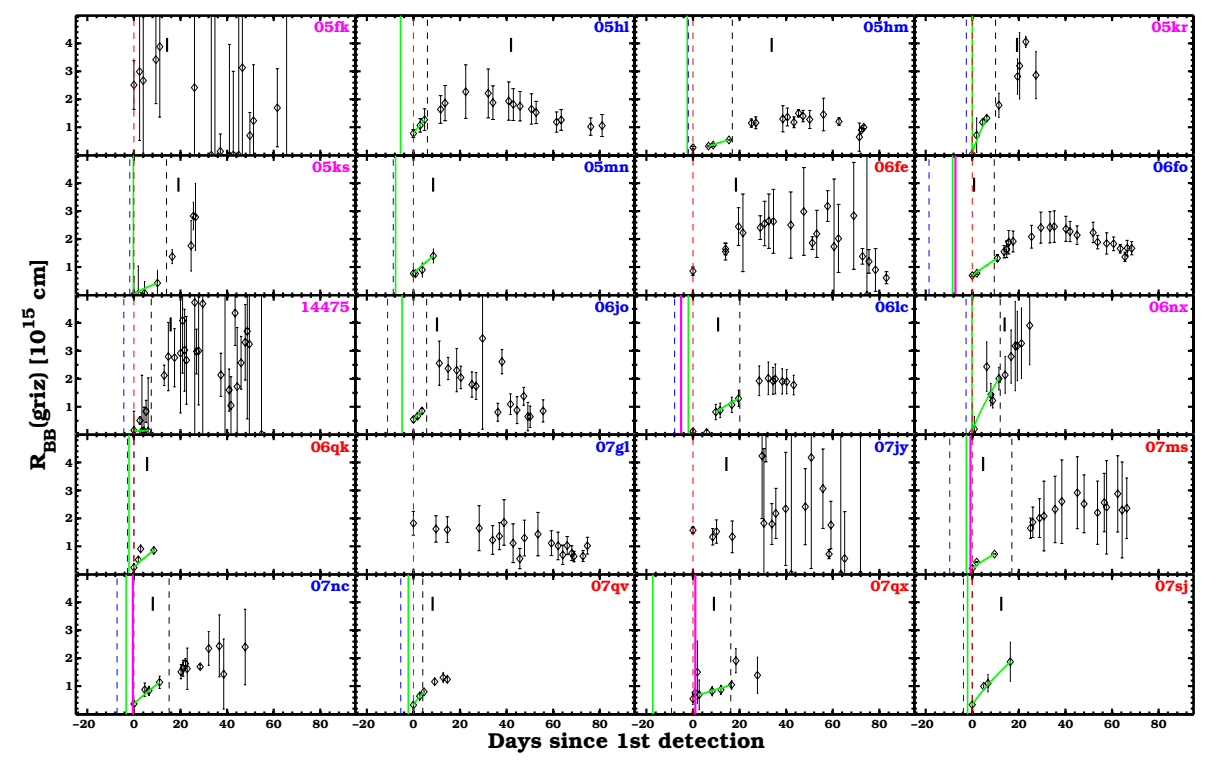

Fig. 20. Photospheric radius evolution for the 20 SDSS $\mathrm{SNe} \mathrm{Ib} / \mathrm{c}$ from the $\mathrm{BB}$ fits on the griz. SEDs. Discovery epochs are marked by red dashed lines, the last non-detections by dashed blue lines. The epoch of the first spectrum is marked by a black segment. Here we include host-extinction corrections. The vertical green line indicates the explosion date as derived from the best PL fit $\left(t_{\text {expl }}^{\mathrm{PL}}\right.$, see Sect. 3.4.1) on the prepeak BB radii, which is shown with a green solid line. The PL-derived explosion dates show that most of the $\mathrm{SNe}$ were discovered at early epochs, when the photospheric radius was remarkably small, and thus that the ${ }^{56} \mathrm{Ni}$ is likely to be present in the outer layers for most of them (see Fig. 1 in PN13 and Sect. 3.4.4). The vertical magenta line indicates the minimum explosion day $t_{\min }$ computed from the spectral velocities (see Sect. 3.4.1). Both $t_{\min }$ and maximum luminosity epochs are marked by black vertical dashed lines. Blue, red, and magenta labels correspond to $\mathrm{SNe} \mathrm{Ib}$, Ic, and Ic-BL, respectively. redder bands. Most of the objects show past-peak temperatures of $\sim 5000 \mathrm{~K}$.

When we do not consider host-extinction correction, $T_{\mathrm{BB}}^{\max }$ ranges from 5500 to $11000 \mathrm{~K}$, with SNe Ic and Ic-BL that appear hotter than $\mathrm{SNe} \mathrm{Ib}$. The range of temperatures can be either due to intrinsic differences and/or to different unaccounted reddening in the host galaxy. We assumed that the colou difference after maximum light is due to the host extinction to compute $E(B-$ $V)_{\text {host }}$ (see Sect. 3.3.1).

\subsubsection{Photospheric radius}

The BB fits on the griz SEDs (corrected for the host extinction) combined with the known distances also allow an estimate of the photospheric radius evolution (Fig. 20). The radius linearly expands at early times, reaches a maximum, then slowly decreases. This behavior is remarkably similar for all the objects. The maximum radius is reached around 20 days after peak luminosity for most of the events. Photospheric radii at the epoch of maximum light $\left(R_{\mathrm{BB}}^{\max }\right)$ have typical values of $10^{15} \mathrm{~cm}$. The results on the radius are not significantly affected by the hostextinction corrections.

When comparing $R_{\mathrm{BB}}^{\max }$ for the different $\mathrm{SN}$ types, $\mathrm{SNe}$ Ic tend to have a typical $R_{\mathrm{BB}}^{\max }$ that is slightly smaller than those of $\mathrm{SNe} \mathrm{Ib}$ and Ic-BL (lefthand panel of Fig. 21). Given that the rise times of SNe Ic and Ic-BL are typically shorter than those of SNe Ib, this result implies that the photospheric velocities of $\mathrm{SNe} \mathrm{Ib}$ and Ic are similar at maximum light (although the uncertainties are large, mainly owing to the error on the explosion date), whereas the same line of reasoning demonstrates that the photospheric velocities of SNe Ic-BL must be considerably higher (see the righthand panel of Fig. 21). The latter is of course expected since SNe Ic-BL have higher spectroscopical expansion velocities by definition.

We compared this result with the velocities that we measured from the spectra (from Si II $\lambda 6355$ and He I $\lambda 5876$ absorption minima), which are reported in Table 2. A better approximation for the photospheric velocity would be what is obtained from the absorption minimum of Fe II $\lambda 5169$ (Branch et al. 2002). However, given the quality of the spectra, it is difficult to perform this measurement. For a few poor-quality spectra, we did
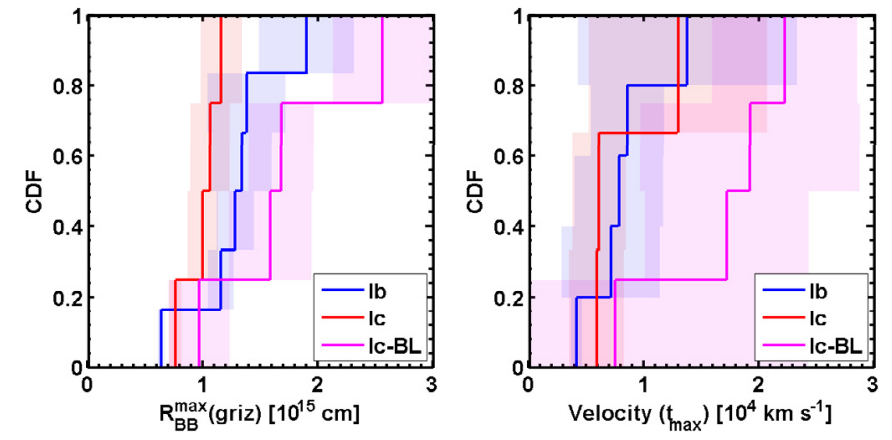

Fig. 21. Cumulative distributions for the photospheric radii and the photospheric velocities at maximum light of SNe Ib, Ic, and Ic-BL. Photospheric velocities are estimated from the photospheric radii divided by the bolometric light curve rise times. Here we include host-extinction corrections. Shaded areas indicate the uncertainty for each SN.

not manage to properly measure the expansion velocity at all. Our spectral measurements confirm that $\mathrm{SNe}$ Ic-BL show higher velocities than $\mathrm{SNe} \mathrm{Ib}$ and $\mathrm{SNe}$ Ic. We do not find any clear difference between SN Ib and Ic expansion velocities, but this spectral sample is not well suited to such a comparison, given the small number of high-quality spectra and the different phases at which the spectra were obtained.

\subsection{Progenitor and explosion properties}

The SDSS-II SN dataset allows us to derive (or at least place limits on) the properties of the SN progenitors and of the explosion parameters, based on the comparison between observed light curves and simple theoretical models at the photospheric phase.

\subsubsection{Explosion dates}

The assumption on the explosion date strongly affects the estimates of the SN progenitor properties, as shown by PN13 in the case of PTF10vgv. A precise knowledge of the time of explosion is useful for obtaining information about the ${ }^{56} \mathrm{Ni}$ mixing in the 


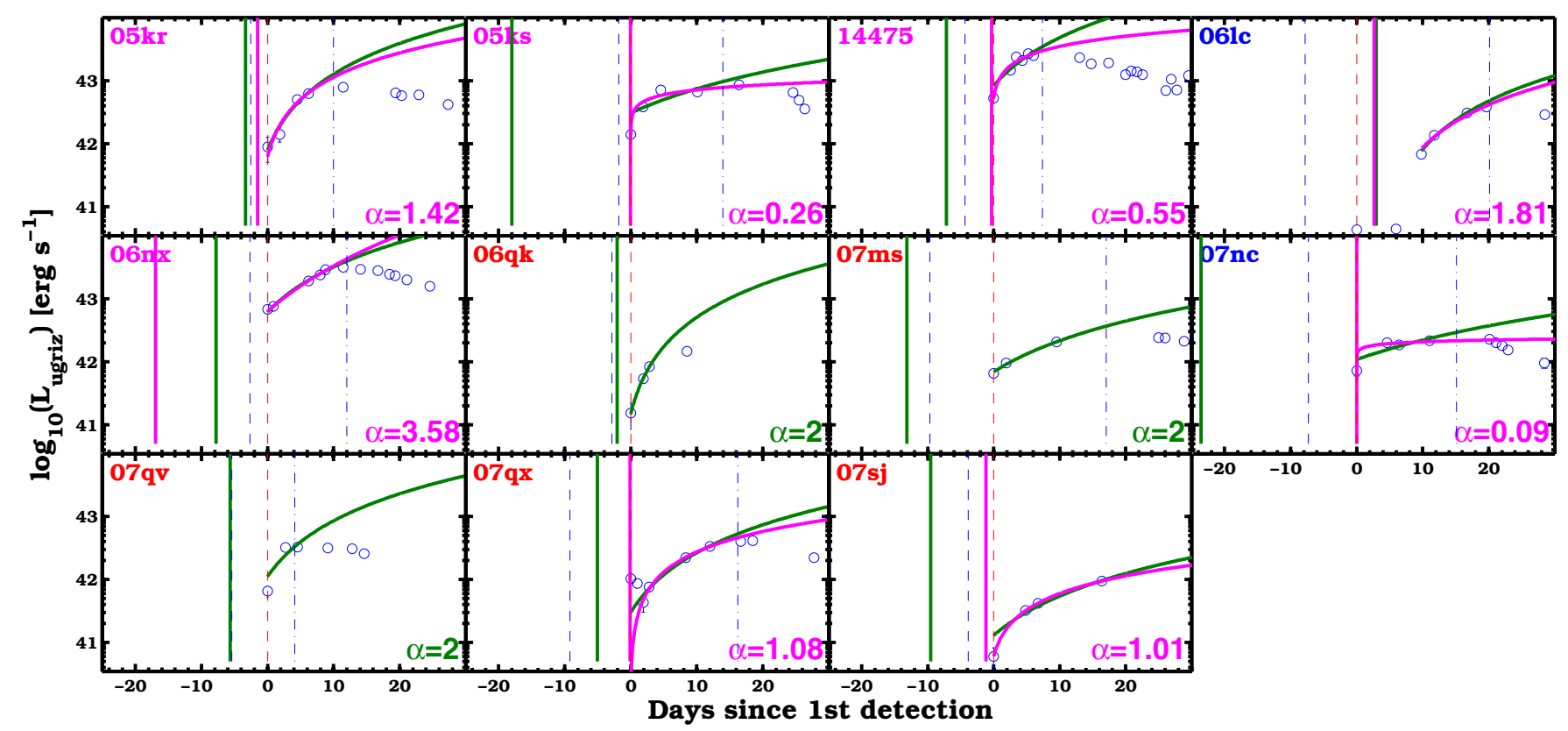

Fig. 22. Power-law (PL) fits on the early part of the pseudo-bolometric light curves of 11 SDSS SNe Ib/c. Here we correct for host extinction. Green solid lines correspond to $\alpha=2$, magenta solid lines to $\alpha$ as a free parameter, whose value is reported in each subpanel. Vertical green and magenta lines indicate the inferred explosion date from the PL fit. Blue and red dashed lines indicate the epochs of last non-detection and first detection, respectively. Black dashed-dotted lines indicate the epoch of maximum light. Blue, red, and magenta labels belong to SNe Ib, Ic, and Ic-BL, respectively.

$\mathrm{SN}$, as well as putting strong limits on the pre-SN radius. Also other physical properties such as ${ }^{56} \mathrm{Ni}$ mass, explosion energy, and ejecta mass can be obtained with higher precision if the explosion date is well established, since their estimates depend on the rise time. We discuss several methods here for estimating the explosion epoch.

The most straight-forward approach to estimating the explosion date of a $\mathrm{SN}$ is to take the average between the first detection and the last non-detection epochs and the uncertainty as half of the time interval determined by these two measurements. This is the explosion date used throughout this paper (coined $\left.t_{\text {expl }}\right)$. The precision of this method is largely affected by the cadence for the light curve sampling. With the cadence of the SDSS-II SN survey, this method implies a typical uncertainty on the explosion epoch of $\pm 2-3$ days for our SN sample. This uncertainty is small enough to exclude the existence of an initial long plateau (Dessart et al. 2011) in the bolometric light curves (see Sect. 3.4.4). Also, the prediscovery images must be deep enough to ensure that the SN was not missed simply because it was too faint. We discuss this aspect in Sect. 3.4.4, where it is shown that, for most of our targets, the limits on the luminosity of the last non-detection are deep enough to exclude a dark plateau phase in the predicted luminosity range.

Another possible way to define the explosion date is to extrapolate the light curve back in time when assuming some functional form, usually a power law (PL), $L(t)=A\left(t-t_{\mathrm{expl}}^{\mathrm{PL}}\right)^{\alpha}$. Cao et al. (2013) applied a PL fit to the early light curve of iPTF13bvn, and the same was done by Pignata et al. (2011) for SN 2009bb. Both PL and broken PL fits have also been used to determine the explosion dates of SNe Ia (e.g., SN $2011 \mathrm{fe}$ and SN 2014J, Nugent et al. 2011; Zheng et al. 2014; Goobar et al. 2014). Different PL shapes have been used to fit earlytime light curves of SE SNe. This approach assumes that the explosion coincides with the beginning of the light curve rise and therefore disregards any dark plateau phase after collapse and before the light curve rise. We designate the power-law extrapolated explosion day $t_{\mathrm{expl}}^{\mathrm{PL}}$. Figure 22 shows our best PL fits to the early time bolometric light curves of those SNe that have enough data points before maximum light. We adopt $\alpha=2$ (consistent with homologous expansion, e.g., Arnett 1982) when we only have three points on the rise, whereas we allow $\alpha$ to be a free parameter when we have at least four points on the rise (corresponding $\alpha$ values are reported in Table 5). The resulting $t_{\text {expl }}^{\mathrm{PL}}$ from the best fits with $\alpha$ as a free parameter are consistent with the explosion dates $t_{\text {expl }}$ estimated in this paper. The lines that indicate the derived $t_{\mathrm{expl}}^{\mathrm{PL}}(\alpha)$ always fall between the last nondetection and the first detection, except in the case of SN 2006nx and, as expected, in that of SN 2006lc, where an early plateau is detected (see Sect. 3.4.4). Conversely, when $\alpha \equiv 2$, we obtain too early explosion dates. The lines that indicate $t_{\text {expl }}^{\mathrm{PL}}(\alpha=2)$ mark explosion dates prior to the last non-detection epoch in most cases.

The SDSS-II SN survey's multi-band coverage allowed us to derive temperatures and radii from the BB fit in Sects. 3.3.4 and 3.3.5. This information can also be used to place additional constraints on the explosion date. PN13 (their Eq. (11)) estimate the photospheric radius $\left(r_{\mathrm{ph}}\right)$ evolution as $\propto\left(t-t_{\mathrm{expl}}^{r_{\mathrm{ph}}=0}\right)^{0.78}$. Assuming that the photospheric radius is similar to the BB radius (basically assuming a constant dilution factor), we fit a PL with $\alpha=0.78$ to the prepeak BB radii presented in Fig. 20, in order to derive the explosion epoch for each $\mathrm{SN}$. We designate the explosion epoch derived from the PL fit with $\alpha=0.78$ of the $\mathrm{BB}$ radius evolution as $t_{\mathrm{expl}}^{r_{\mathrm{ph}}=0}$. For all SNe, $t_{\mathrm{expl}}^{r_{\mathrm{ph}}=0}$ occurs between the last non-detection and the first detection (see Fig. 20, where the green vertical line is always included between the vertical blue and red lines), matching our simplest explosion date $\left(t_{\text {expl }}\right)$ estimates. The same result is obtained when we fit the BB radii from the host-extinction uncorrected SEDs. 
We also use the spectral information at the epoch of the rise for some of our SNe in order to determine the minimum time of explosion, $t_{\min }$ as defined by PN13 in their equation 17 . From the spectra we derive the velocity (see Table 2 ), whereas the temperature $(T)$ and bolometric luminosity $(L)$ was obtained by interpolating $T_{\mathrm{BB}}$ and $R_{\mathrm{BB}}$ at the spectral epoch and then assuming $\mathrm{BB}$ emission. For five events, $t_{\min }$ always occurs after the last non-detection and before discovery (Fig. 20). This is true regardless of the host-extinction corrections. The estimates of $t_{\min }$ linearly depend on the assumed velocity, and the spectral velocities are only an approximation of the actual photospheric velocity. However, we find that $t_{\min }$ occurs between last non-detection and discovery for the vast majority of our sample also when a variation in velocity of $\pm 20 \%$ is allowed.

We conclude that the $t_{\text {expl }}$ estimates presented in Sect. 3.1 and based on the first method presented in this section are in good agreement with the explosion dates inferred from the model for the evolution of the photospheric radius $\left(t_{\mathrm{expl}}^{r_{\mathrm{ph}}=0}\right)$ and with $t_{\mathrm{min}}$ (PN13). Therefore the rise times shown in Fig. 10 should also correspond to the actual rise times, defined as the time interval between explosion and peak. This analysis also suggests that a long early plateau phase is not common in $\mathrm{SNe} \mathrm{Ib} / \mathrm{c}$, since the light curves tend to rise soon after explosion (see Sect. 3.4.4).

\subsection{2. ${ }^{56} \mathrm{Ni}$ mass, explosion energy and ejecta mass}

The amount of ${ }^{56} \mathrm{Ni}$ synthesized during the explosion, the ejecta mass $\left(M_{\mathrm{ej}}\right)$, and the explosion energy $\left(E_{\mathrm{K}}\right)$ of our SNe can be estimated by fitting the Arnett model (Arnett 1982) for SNe I (see, e.g., Eq. (1) in Cano 2013) to their bolometric light curves. This model can be applied in the photospheric phase, which is the case for our sample. Therefore we built optical+near-infrared (NIR) bolometric light curves by adding a near-infrared contribution to our host-extinction-corrected quasi-bolometric light curves (see Fig. 16 and Sect. 3.3.3). This contribution is computed from the fraction of NIR $(\lambda>9000 \AA)$ BB flux to the total BB flux (redward of $3500 \AA$ ) given the BB temperatures computed in Sect. 3.3.4 and shown in Fig. 18. At early times this correction is small for most SNe (the optical flux is $\sim 70-80 \%$ ), whereas after maximum light, the NIR contribution rises in importance as the temperature decreases. We also fixed the $E_{\mathrm{K}} / M_{\mathrm{ej}}$ ratio for each event by using the photospheric velocities at maximum light $\left(v_{\mathrm{ph}}^{2}=\frac{10}{3} E_{\mathrm{K}} / M_{\mathrm{ej}}\right.$, assuming the $\mathrm{SN}$ ejecta to be an expanding sphere with constant density) computed in Sect. 3.3.5 and shown in the righthand panel of Fig. 21. In the Arnett formula we adopted ${ }^{56} \mathrm{Ni}$ and ${ }^{56} \mathrm{Co}$ decay times $\left(\tau_{\mathrm{Ni}}\right.$ and $\left.\tau_{\mathrm{Co}}\right)$, energy releases per second per gram $\left(\epsilon_{\mathrm{Ni}}\right.$ and $\left.\epsilon_{\mathrm{Co}}\right)$, opacity $(\kappa=$ $0.07 \mathrm{~cm}^{2} \mathrm{~g}^{-1}$, which is appropriate when the electron scattering dominates Chevalier e.g., 1992) and constant of integration $(\beta)$ as in Cano (2013). The assumed explosion dates $\left(t_{\text {expl }}\right)$ for each SN were those estimated and discussed in Sect. 3.4.1.

The best fits to the bolometric light curves (shown in Fig. 16) give the values for ${ }^{56} \mathrm{Ni}$ mass, $M_{\mathrm{ej}}$, and $E_{\mathrm{K}}$ that are listed in Table 6. The quoted uncertainty is due to the precision on the explosion date estimate. When we compare SNe Ib and Ic, we do not find any statistically significant difference in the amount of ejected ${ }^{56} \mathrm{Ni}$ (Fig. 23, lefthand panel). On the other hand, SNe Ic$\mathrm{BL}$ appear richer in ${ }^{56} \mathrm{Ni}$ than both $\mathrm{SNe} \mathrm{Ib}$ and Ic. When we compare (see Fig. 23) $M_{\text {ej }}$ among SNe Ib, Ic, and Ic-BL, we do not find any statistically significant difference. (We only have a few $\mathrm{SNe} I c$, and the uncertainties are quite large.) However, SNe Ic$\mathrm{BL}$ and $\mathrm{SNe} \mathrm{Ic}$ appear slightly more massive than $\mathrm{SNe} \mathrm{Ib}$. The comparison of $E_{\mathrm{K}}$ shows that this parameter is clearly larger for
SNe Ic-BL than for SNe Ic and Ib. We compare our best estimates to those found by Drout et al. (2011) and Cano (2013) in Sect. 4.

\subsubsection{Early plateau phase}

The ${ }^{56} \mathrm{Ni}$ mass estimated in Sect. 3.4.2 can either be mixed out in the ejecta to the outermost layers, or be distributed more centrally. The mechanism that brings ${ }^{56} \mathrm{Ni}$ near the ejecta surface is not clearly established, but jets might be a possible explanation (e.g., Maund et al. 2007a,b; Maund et al. 2009; Couch et al. $2009,2011)$. We note that the use of the word "mixing" to define the presence of ${ }^{56} \mathrm{Ni}$ in the outermost ejecta might erroneously suggest that this is the effect of small scale, quasi-homogeneous mixing, rather than large-scale plumes. Therefore, to study the ${ }^{56} \mathrm{Ni}$ mixing is important for understanding the explosion mechanism of SN progenitors.

The different degrees of mixing should affect the shape of the light curve, in particular in the early phases. For instance, the presence of an initial, low-luminous plateau between the shock breakout cooling tail and the rise of the light curve was noted as an outcome from hydrodynamical models of compact stars by Dessart et al. (2011, their Fig. 1). They found that the duration of the initial plateau was around ten days with a luminosity $L_{\mathrm{p}}=10^{40.6-41.3} \mathrm{erg} \mathrm{s}^{-1}$. Such a long plateau phase was produced because the ${ }^{56} \mathrm{Ni}$ was assumed to be located close to the centre. PN13 present a discussion (and a semi-analytical formula that describes the luminosity and duration of this early plateau), which illustrates that a deep ${ }^{56} \mathrm{Ni}$ distribution leads to a long (a few days) early plateau, whereas a shallow (strongly mixed) ${ }^{56} \mathrm{Ni}$ distribution produces a light curve that rises soon after shock breakout. Bersten et al. (2013) confirm the presence of an initial plateau in compact stars and studied the effect of ${ }^{56} \mathrm{Ni}$ mixing applied to the particular case of SN 2008D. That analysis concludes that ${ }^{56} \mathrm{Ni}$ mixing is the main factor that determines the plateau duration. Figure 24 shows some results for the early plateau properties, which were obtained with the hydrodynamical code of Bersten et al. (2011). We calculated a set of light curves for two initial models with ejecta masses and progenitor radii of $1.7 M_{\odot}, 2.5 R_{\odot}$, and $2.2 M_{\odot}, 2.3 R_{\odot}$, respectively. We adopted a ${ }^{56} \mathrm{Ni}$ mass of $0.2 M_{\odot}$ in all the calculations. For each model we assumed four different degrees of ${ }^{56} \mathrm{Ni}$ mixing, $0.60,0.83,0.95$, and 1.00 of the progenitor mass $\left({ }^{56} \mathrm{Ni}\right.$ is linearly distributed in mass coordinates, see Bersten et al. 2012) and three different explosion energies, $E_{\mathrm{K}}=1,1.5$, and 2 foe ( 1 foe $=10^{51} \mathrm{erg}$ ). These assumed values for the ${ }^{56} \mathrm{Ni}$ mass, $E_{\mathrm{K}}$, and $M_{\mathrm{ej}}$ are consistent with our findings in Sect. 3.4.2 and with those available in the literature for $\mathrm{SNe} \mathrm{Ib/c} \mathrm{(Drout} \mathrm{et} \mathrm{al.}$ 2011; Cano 2013). All models predict an initial plateau whose duration depends mainly on the mixing of ${ }^{56} \mathrm{Ni}$ (lefthand panel of Fig. 24), with short plateaus for strongly mixed models and long-duration plateaus for models with weak ${ }^{56} \mathrm{Ni}$ mixing. The early plateau duration range is around two to ten days, with durations shorter than three to four days, implying an almost full ${ }^{56} \mathrm{Ni}$ mixing ( $\left.\gtrsim 83-95 \%\right)$. The luminosity of the plateau depends mainly on the explosion energy: the higher the energy, the brighter the plateau. There is also dependence on the ejecta mass: the higher the mass, the fainter the plateau. The difference in magnitude between peak and plateau luminosity $(\Delta M)$ is large for low energy and/or for high mass. In our models, the predicted range of plateau luminosity is $\sim 10^{41.2-41.8} \mathrm{erg} \mathrm{s}^{-1}$ (central panel of Fig. 24), and the $\Delta M$ range is $2.3-3.4$ mag (righthand panel of Fig. 24). 

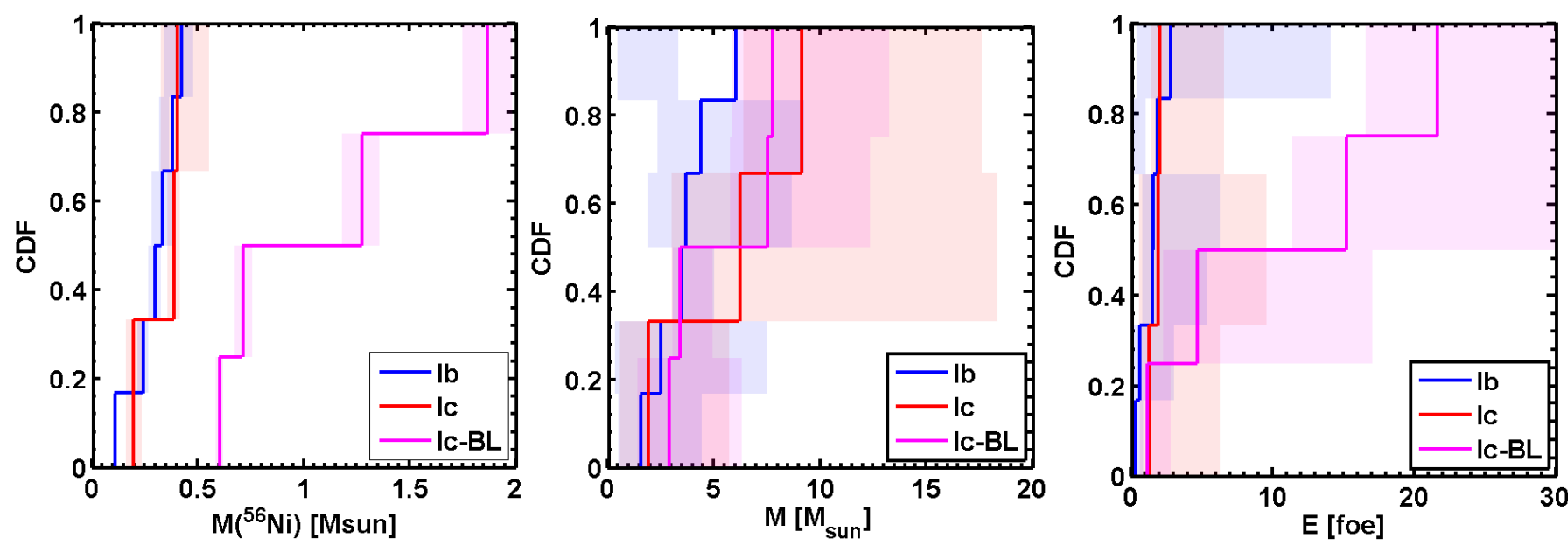

Fig. 23. Lefthand panel: ${ }^{56} \mathrm{Ni}$ mass cumulative distributions for $\mathrm{SNe} \mathrm{Ib}$, Ic, and Ic-BL observed at peak. The comparison reveals higher ${ }^{56} \mathrm{Ni}$ masses for SNe Ic-BL (host extinction included). Shaded areas indicate the uncertainties for each SN, caused by the error on the explosion day. Central panel: ejecta mass (M) cumulative distributions for SNe Ib, Ic, and Ic-BL. SNe Ic and Ic-BL appear more massive than SNe Ib, but the uncertainties are large. Righthand panel: explosion energy $\left(E_{\mathrm{K}}\right)$ cumulative distributions for $\mathrm{SNe} \mathrm{Ib}$, Ic, and Ic-BL. SNe Ic-BL are clearly more energetic. We compare our results to those of Cano (2013) and Drout et al. (2011) in Sect. 4.1.
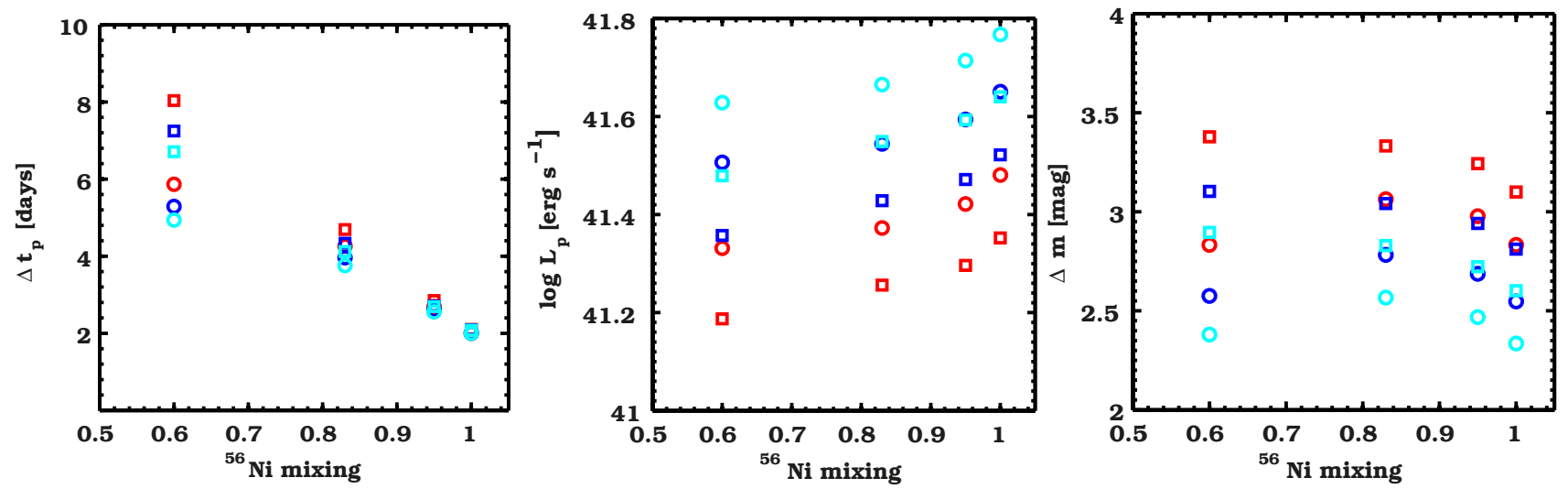

Fig. 24. Plateau length $\left(\Delta t_{\mathrm{p}}\right)$, luminosity $\left(L_{\mathrm{p}}\right)$, and difference in magnitude $(\Delta M)$ between $L_{\mathrm{p}}$ and the luminosity peak as functions of ${ }^{56} \mathrm{Ni}$ mixing $\left({ }^{56} \mathrm{Ni}\right.$ mass is mixed out to $0.60,0.83,0.95$, and 1.00 of the total progenitor mass). Red, blue, and cyan symbols correspond to models with $E=1,1.5,2$ foe, respectively. Circles and squares correspond to models with ejecta mass and progenitor radius $M_{\mathrm{ej}}=1.7 / R=2.5 R_{\odot}$ and $2.2 M_{\odot} / R=2.3 R_{\odot}$, respectively.

\subsection{4. ${ }^{56} \mathrm{Ni}$ mixing}

Since the early plateau phase is such an important diagnostic for the amount of ${ }^{56} \mathrm{Ni}$ mixing, we studied the quasi-bolometric light curves of the SDSS SNe Ib/c to search for evidence of this phase. Since we did not find any clear early plateau emission except in a single case (see below), we examined further whether our observations had deep enough limits on luminosity and high enough cadence to actually detect this plateau.

Given the expected temperature at the plateau phase ( 7000-8000 K, Dessart et al. 2011), the quasi-bolometric (optical) luminosities should range between $\sim 10^{41.0-41.6} \mathrm{erg} \mathrm{s}^{-1}$ (assuming BB emission and the bolometric luminosity range from our models). Table 7 reports the last non-detection luminosity $1 \sigma$ limits $\left(\Delta L_{\text {last non-det. }}\right)$. It is clear that we would have been able to detect a plateau at the expected luminosity range in most of our objects, since most of the detection limits are lower than the expected values provided by the models ( 7 out of 13 are $\leq 10^{41} \mathrm{erg} \mathrm{s}^{-1}$ ).

We can also put limits on the plateau duration $\left(\Delta t_{\mathrm{p}}\right.$, see Table 7), assuming this is equal to the time interval between the last non-detection (which is shown to be robust, see Sect. 3.4.1) and the discovery of the $\mathrm{SN}$, as exemplified in the lefthand panel of Fig. 25 for the case of SN 2007ms. In the specific cases of SN 2005hm and SN 2007qx, the limits on the duration of the plateau are set by the time interval between the first(second) and the second(fourth) detections, since these two $\mathrm{SNe}$ were very likely observed at the epoch of the shock breakout cooling tail (see Sect. 3.3.5). Figure 25 also reports the other plateau parameters discussed in this section and presented for each $\mathrm{SN}$ in Table 7. In three cases (SNe 2005ks, 2006qk, 2007qx) $\Delta t_{\mathrm{p}}<2$ days. Two of these objects are SNe Ic, and the other is a SN Ic-BL. Two additional SNe Ic-BL (2005kr, 2006nx) show $\Delta t_{\mathrm{p}}<3$ days, SN Ic 2007sj exhibits $\Delta t_{\mathrm{p}}<4$ days, and the SN Ic-BL 14475 has a plateau duration that can be constrained to $\Delta t_{\mathrm{p}}<4.4$ days. We thus conclude that within the context of the aforementioned hydrodynamical models, five SNe must have ${ }^{56} \mathrm{Ni}$ mixed into the outermost layers $\left(\Delta t_{\mathrm{p}}<3\right.$ days $)$, and that two more objects show strong ${ }^{56} \mathrm{Ni}$ mixing (above $\sim 83 \%$ of the progenitor mass). All of these events appear to be helium-poor.

We do not have equally tight constraints on the plateau duration for $\mathrm{SNe} \mathrm{Ib}$, whose shortest limit is $\Delta t_{\mathrm{p}}<6.8$ days (SN 2005hm). For SN Ib 2006lc we can actually observe what we interpret to be an early plateau phase, at faint luminosity 

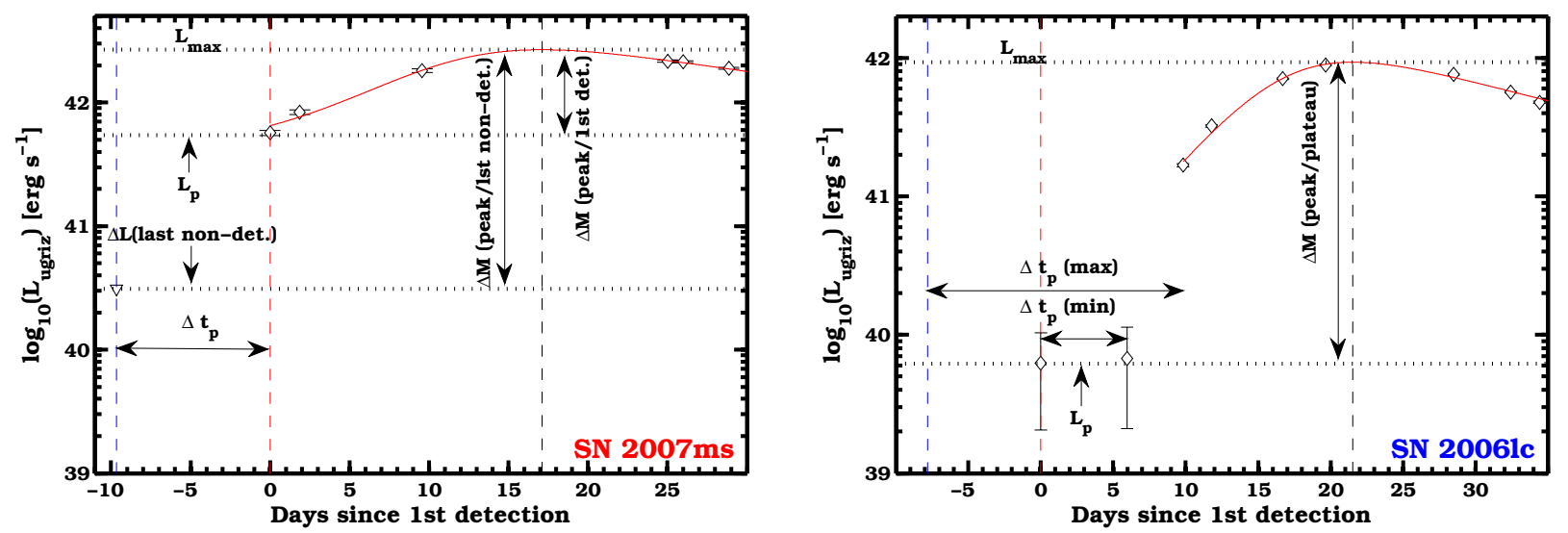

Fig. 25. Early-time pseudo-bolometric light curves (host extinction neglected) of SN 2007ms and SN 2006lc, where we label the parameters discussed in Sect. 3.4.3.

(see the righthand panel of Fig. 25) and $T_{\mathrm{BB}}$ temperature of $8000 \mathrm{~K}$ (with host-extinction correction). We have two detections at $\sim 81.3 \times 10^{39} \mathrm{erg} \mathrm{s}^{-1}$, i.e., at 4.2 mag below peak luminosity. The duration of this plateau ranges from a minimum of $\Delta t_{\mathrm{p}}(\mathrm{min}) \approx 5.9$ days (the interval between the two detections) to a maximum of $\Delta t_{\mathrm{p}}(\max ) \approx 17.6$ days (last non-detection epoch to first epoch on the rise). These results would imply a low degree of ${ }^{56} \mathrm{Ni}$ mixing for SN 2006lc, lower than $\sim 60 \%$ of the progenitor mass.

We also take this opportunity to review the few examples in the literature for which we know the explosion date with considerable accuracy and to investigate whether there is evidence for any early and faint plateau phase in these objects. Our knowledge of the time of explosion depends on the detection of the shock breakout or of its cooling phase. SN 2008D (Soderberg et al. 2008; Malesani et al. 2009; Cowen et al. 2010) presents $B V R I$ light curves at early times with a possible plateau lasting for around four days. SN 1999ex (Stritzinger et al. 2002), a SN Ib, shows an early dip in the optical light curves. There is no clear plateau phase between the early light curve declining phase and the rise: any plateau can therefore not be longer than $\lesssim 2-3$ days. Two other SNe with good pre-explosion limits where the plateau is undetected are SN Ic PTF10vgv (Corsi et al. 2012) and SN Ic-BL 2012gzk (Ben-Ami et al. 2012). In the case of SNe associated to GRBs, such as SN 1998bw (Galama et al. 1998) and SN 2006aj (Sollerman et al. 2006; Ferrero et al. 2007; Cowen et al. 2010), the early afterglow light could mask the possible plateau phase. However, these events are probably highly asymmetric explosions (Maeda et al. 2008), and we do expect significant mixing of radioactive nickel in such cases, hence a short or non-existent plateau phase as in the stronglymixed models by Dessart et al. (2011). In summary, SNe Ib/c with known explosion dates in the literature show short $(\lesssim 2-4)$ limits on the plateau duration, again suggesting that their ${ }^{56} \mathrm{Ni}$ masses are strongly mixed in the ejecta.

The rising part of the light curves can also provide important information on the ${ }^{56} \mathrm{Ni}$ mixing. Within the context of the simple models provided by PN13, if we fit a PL $\left[L(t)=A\left(t-t_{\text {expl }}^{\mathrm{PL}}\right)^{\alpha}\right]$ to the rising part of the light curves and find an index $\alpha<2$, this would mean that the direct radioactive heating dominates, with the ${ }^{56} \mathrm{Ni}$ significantly mixed out in the ejecta. On the other hand, when the light curve is steeper than $\alpha=2$, this is interpreted by PN13 as a diffusive ${ }^{56} \mathrm{Ni}$ tail affecting its shape, owing to the central location of the ${ }^{56} \mathrm{Ni}$. The contribution of the radioactive diffusive tail is as important as the direct radioactive heating until 9-12 days past explosion, after which it progressively vanishes as the light curve reaches the peak (see Fig. 8 in PN13). Since it is crucial to have early observations to constrain the index $\alpha$, we have fit PLs with $\alpha$ as a free parameter only to those bolometric light curves where the first observed luminosity is at least 2 mag below maximum light. The fit was performed on all the prepeak epochs of the bolometric light curves. From Fig. 22 it is clear that $\alpha=2$ is too steep to reproduce the early shape, and it also implies too early an explosion date. (The suggested explosion would occur even ten days before the last non-detection for some SNe.) The objects that were fit with $\alpha$ as a free parameter indicate that $\alpha$ is lower than 2, spanning $0.09<\alpha<1.42$. The exceptions are SN 2006lc and SN 2006nx, whose early light curves are reproduced by $\alpha \gtrsim 2$. In the context of the model by PN13, this means that the ${ }^{56} \mathrm{Ni}$ is mixed throughout the ejecta in most of these objects. These conclusions also hold when we include the host-extinction corrections on the bolometric light curves. A PL fit to the early light curve (first 4 days) of iPTF13bvn by Cao et al. (2013) gave $\alpha=1.54$, suggesting that the direct ${ }^{56} \mathrm{Ni}$ radioactive heating dominates.

The temperature profiles in Figs. 18 and 19 confirm the ${ }^{56} \mathrm{Ni}$ mixing in the outer layers of most of our objects. PN13 show that the temperature is expected to increase during the light curve rise if the ${ }^{56} \mathrm{Ni}$ is centrally located, whereas it should stay roughly constant, or even drop, if the ${ }^{56} \mathrm{Ni}$ is distributed more uniformly. In our $\mathrm{SNe}$, we always observe constant or decreasing $\mathrm{T}_{\mathrm{BB}}$ at the prepeak epochs, with the exception of SN 2006lc, which is confirmed to have a steep ${ }^{56} \mathrm{Ni}$ distribution.

In the literature, Takaki et al. (2013) and Cano et al. (2014) have recently estimated a high degree of ${ }^{56} \mathrm{Ni}$ mixing for $\mathrm{SNe} \mathrm{Ib}$ 2012au and 1999dn, respectively.

\subsubsection{Progenitor radius}

We can also use the analytic model by PN13 for the early bolometric light curve plateau (see their Eq. (5)) to put limits on the progenitor radius. The luminosity at the discovery epoch is used as an upper limit to the plateau luminosity, making a conservative assumption. We furthermore use values for $E_{\mathrm{K}}$ and $M_{\mathrm{ej}}$ derived in Sect. 3.4.2 and listed in Table 6 (for the SNe lacking $E_{\mathrm{K}}$ and $M_{\mathrm{ej}}$ estimates, the average values of their spectral class were used).

We take into account that our derived bolometric luminosities only constitute a fraction of the total bolometric luminosities ( $\sim 60 \%$ if we assume a plateau temperature of $0.6 \mathrm{eV}(6959 \mathrm{~K})$ 
and BB emission as in PN13), since we integrated between 3500 and $9000 \AA$. The corresponding limits on the progenitor radii are listed in Table 6 . Including host-extinction corrections, for $\mathrm{SNe}$ Ic, Ic-BL, and Ib, the tightest upper limits are of 1.6, 6.0, and $1.7 R_{\odot}$. In the case of SN 2006lc, where we argue that something similar to the plateau phase is indeed detected, it is necessary to include the large and uncertain host-extinction correction in order to derive a reasonable radius. The main conclusion that we obtain from the SNe with the best limits on the plateau luminosity is that these transients arise from compact progenitors, with a size of no more than a few solar radii.

We stress that the limits on the progenitor radius are obtained in the framework of the analytic model by PN13, where the plateau luminosity is fixed to the specific value of the bolometric luminosity powered by the shock cooling when the temperature reaches $0.6 \mathrm{eV}$. The strong dependence of $L_{\mathrm{p}}$ on the progenitor radius is not reproduced by hydrodynamical models (Bersten et al. 2014). In the future surveys (e.g., ZTF) we will be able to systematically detect the early emission from the shock cooling for $\mathrm{SNe} \mathrm{Ib} / \mathrm{c}$ and will have better constraints on the explosion dates. These achievements will imply more accurate radius estimates.

Two objects, SNe 2007qx and 2005hm, show hints of the cooling that follows the shock breakout in their early light curves. An early dip in the light curves was detected for SN 2007qx, which suggests that the explosion of this SN occurred immediately before the first detection. The dip is clearly visible in the bluer bands and less pronounced in the redder ones, consistent with an initial cooling phase, as observed in, for example, SN 1999ex (Stritzinger et al. 2002). Recently Nakar \& Piro (2014) have shown that the appearance of an early maximum in the bluer optical bands, and the absence of it in the redder ones can be explained if the progenitor star has most of its mass next to the hydrostatic stellar radius, in an envelope whose mass $M_{\text {ext }}$ is higher than that of the core $M_{\text {core }}$. It is possible to estimate the progenitor radius of this object by fitting the first two points of the quasi-bolometric light curve with the model described in equations 1 and 2 of PN13, assuming $E_{\mathrm{K}}$ and $M_{\mathrm{ej}}$ from the Arnett model fit presented in Sect. 3.4.2. The quasi-bolometric luminosity model is derived from the total bolometric luminosity and the temperature profile by assuming $\mathrm{BB}$ emission and considering only the fraction of flux emitted in the wavelength range 3500-9000 $\AA$. The analysis indicates that the explosion of SN 2007qx occurred 5.5 days before first detection and that the progenitor radius is $16-32 R_{\odot}$, including host-extinction corrections.

In the case of SN 2005hm, we observe a rather high luminosity at the first epoch (only 2 days after the last non-detection) if compared to the second epoch, which is already clearly on the rising part of the light curve (see Fig. 8). Unlike SN 2007qx, the first epoch is bright in all the optical bands. This behavior is reminiscent of the early light curve of SN 2008D (Soderberg et al. 2008; Malesani et al. 2009; Modjaz et al. 2009), where a bright cooling phase dominated the light curve for about five days. To explain the fist peak, Bersten et al. (2013) proposed a scenario where a jet produced during the explosion has deposited ${ }^{56} \mathrm{Ni}-$ rich material in the outer layers of the ejecta. Nakar \& Piro (2014) suggest that the presence of an early maximum in the $r$ and $i$ bands might arise from a non-standard stellar progenitor that has an extended low-mass envelope surrounding a more massive core $\left(M_{\text {ext }} \ll M_{\text {core }}\right)$. If we assume that the explosion date occurred exactly between last non-detection and discovery and that our first photometric observation corresponds to the early peak, Eq. (12) in Nakar \& Piro (2014) gives a radius of $\approx 8.3 R_{\odot}$. Here we adopted energy and core mass from Table 6 , and we corrected the quasi-bolometric luminosity of the first peak to take all of the BB emission into account.

With these two objects we confirm that $\mathrm{SN} \mathrm{Ib/c} \mathrm{progenitors}$ are relatively compact.

\section{Discussion}

In this paper we have presented the first study of a large, homogeneous sample of $\mathrm{SNe} \mathrm{Ib} / \mathrm{c}$ observed in five optical passbands. When we compare absolute luminosities and light curve widths to those of the well studied sample of Drout et al. (2011), we find similar trends (see Sects. 3.2.3 and 3.3.2). This suggests that, even though the SDSS-II SN survey was mainly aiming for $\mathrm{SNe} \mathrm{Ia}$, the sample of SDSS SNe Ib/c is not significantly biased, and we can use it to draw conclusions on the nature of $\mathrm{SN} \mathrm{Ib/c}$ progenitors.

\subsection{Implications for the SN progenitors}

Dessart et al. (2011) show in their numerical models that the spectral difference between $\mathrm{SNe} \mathrm{Ib}$ and Ic might be largely due to different mixing. In $\mathrm{SNe} \mathrm{Ib}$, the ${ }^{56} \mathrm{Ni}$ could be mixed out to the outermost layers, where its decay can excite the helium lines that define the Type Ib class. In contrast, in SNe Ic, ${ }^{56} \mathrm{Ni}$ might be more centrally distributed; this would prevent the excitation of any helium layers and thus suppress the appearance of helium lines in their spectra. The different degrees of ${ }^{56} \mathrm{Ni}$ mixing should also have consequences for the light curves, in particular at early times, and thus allow investigation of whether this is the main parameter distinguishing between $\mathrm{SNe}$ Ib and Ic.

We have shown that for our SDSS SNe Ic and for other $\mathrm{SNe}$ Ic in the literature, the limits on the plateau length are consistent with a shallow ${ }^{56} \mathrm{Ni}$ distribution. Also the early light curve shape and the temperature evolution suggest that ${ }^{56} \mathrm{Ni}$ is thoroughly mixed in most of our SNe, including SNe Ic. Moreover, a strongly mixed $\mathrm{SN}$ has a shorter light curve rise time than a poorly mixed one, as reported by Dessart et al. (2012). Since the light-curve rise times are shorter for helium-poor transients than for $\mathrm{SNe} \mathrm{Ib} / \mathrm{IIb}$, one could suspect that mixing is actually stronger in SNe Ic. The lack of mixing of radioactive elements in the outer layers of helium does not appear to cause the difference between $\mathrm{SNe} \mathrm{Ib}$ and SNe Ic. The difference in rise time could also be produced by a larger $E_{\mathrm{K}} / M_{\mathrm{ej}}$ ratio for SNe Ic than for $\mathrm{SNe} \mathrm{Ib}$, but there is no evidence to support this conclusion (see also Cano 2013). We conclude that ${ }^{56} \mathrm{Ni}$ mixing is important for the early light curves of $\mathrm{SNe} \mathrm{Ib} / \mathrm{c}$, but that the difference in degree of mixing does not play a pivotal role in the spectroscopic difference between $\mathrm{SNe} \mathrm{Ib}$ and Ic. The driving factor of this difference is therefore more likely a real lack of helium in SNe Ic (e.g., Wheeler et al. 1987). Also Cano et al. (2014) have recently found a strong mixing for SN $1999 \mathrm{dn}$ and two other $\mathrm{SNe} \mathrm{Ib}$, whereas in the single case of SN Ic $2007 \mathrm{gr}$, the mixing seems to be poor. Another possible explanation for the absence of helium lines in SNe Ic can be found in a strongly asymmetric ${ }^{56} \mathrm{Ni}$ mixing (Dessart et al. 2012) in these SNe, which would produce the absence of helium lines if the $\mathrm{SN}$ is observed under specific viewing angles.

In our work we found that $\mathrm{SNe}$ Ic-BL have more ${ }^{56} \mathrm{Ni}$ mass and higher energy than $\mathrm{SNe} \mathrm{Ib}$ and Ic. These results were also found by (Drout et al. 2011) and Cano (2013). Our estimates for the ${ }^{56} \mathrm{Ni}$ masses are more like those of Drout et al. (2011) 
than those of Cano (2013). For SNe Ib, Ic, and IcBL, we and Drout et al. (2011) found $M\left({ }^{56} \mathrm{Ni}\right)=0.3,0.3$, and $0.6-1.1 M_{\odot}$, whereas Cano (2013) estimated $M\left({ }^{56} \mathrm{Ni}\right)=0.2,0.2$, and $0.4 M_{\odot}$. In terms of ejecta masses, we found $M_{\mathrm{ej}}=3.6,5.7,5.4 M_{\odot}$ for $\mathrm{SNe} \mathrm{Ib}$, Ic and IcBL. The two other works estimated $M_{\mathrm{ej}}=2.0$, 1.7, 4.7 $M_{\odot}$ (Drout et al. 2011) and $M_{\mathrm{ej}}=4.7,4.5,5.4 M_{\odot}$ (Cano 2013). However, the errors on the ejecta masses are large in all the works, and the differences are not significant. Concerning the explosion energy, we obtained $E_{\mathrm{K}}=1.5,1.7,10.7$ foe, whereas Drout et al. (2011) reports $E_{\mathrm{K}}=1.2,1.0,11.0$ foe and Cano (2013) $E_{\mathrm{K}}=3.3,3.3,12.6$ foe; i.e. all the works report similar values (within the errors, see Table 6$)^{14}$.

With the early time light curves, we have been able to constrain the radius of the SN progenitors. Our limits are based on the analytic model of PN13. For the SNe with the earliest observations and the tightest constraints on the plateau luminosity, we found that these objects are compact $(R \lesssim 1.6$ (Ic), 6.0(Ic-BL), 1.7(Ib) $R_{\odot}$ ). For the Type Ib SN 20061c, whose early time plateau was observable, we estimated a radius of $\approx 1.7 R_{\odot}$. For SN Ic $2007 \mathrm{qx}$, the estimate from the possible early shock breakout cooling tail gives a radius of $\approx 16-32 R_{\odot}$. For SN Ib $2005 \mathrm{hm}$, the first peak along with the known energy and mass reveal a radius of $\approx 8.3 R_{\odot}$. These radii agree with the values expected for hot and massive $\left(M_{\text {ZAMS }}>25 M_{\odot}\right)$ Wolf-Rayet stars, which are stripped of their hydrogen-rich envelopes by strong line-driven winds, but also with the radii of stars that were initially less massive and were stripped by a companion (Yoon et al. 2010). In both cases the expected final mass for the progenitors is around $4-8 M_{\odot}$ (the smaller the radius, the higher the final mass). These values are compatible with our findings on the ejecta masses from the Arnett model. SNe IIb, like SN 2011dh, have been found to arise from extended $\left(\sim 200 R_{\odot}\right)$ yellow supergiant progenitors (Maund et al. 2011; Bersten et al. 2012; Ergon et al. 2014, 2015). We note that, using the PN13 model, we have not explored the possibility that, at early times, there is some circumstellar interaction contributing to the light curve. This is not unreasonable because SE SN progenitors should suffer large mass losses before collapse. We have also neglected that a substantial part of the gamma-ray deposition is non-local. This is probably a significant effect when ${ }^{56} \mathrm{Ni}$ is transported to near the surface. Another assumption is the constant opacity, used in the Arnett model.

\subsection{Outlook for ongoing and future surveys (iPTF, ZTF)}

The ongoing iPTF survey and, even more, the future ZTF survey will discover and follow hundreds of new SE CC SNe. These surveys aim to scan large portions of the sky with high cadence in order to find young transients. The same fields will be imaged multiple times per night, obtaining exceptionally dense light curve sampling. The study of the early light curves of SE CC SNe will obviously benefit from these observations.

Given the compact nature of their progenitors, $\mathrm{SNe} \mathrm{Ib/c} \mathrm{are}$ expected to emit a short burst of high-energy (soft $\gamma$-rays, X-ray, see Nakar \& Sari 2010) radiation at the moment of shock breakout, which should last no more than $\sim 15 \mathrm{~min}$. Thereafter, the cooling will bring the emission into the UV-optical range, which

\footnotetext{
14 During the refereeing process, a paper by Lyman et al. (2014) was published with similar results to those of our paper. In particular from their analysis, it emerges that $\mathrm{SNe}$ Ic-BL have higher ${ }^{56} \mathrm{Ni}$ masses and the kinetic energies than those of the other subtypes. Furthermore, $\mathrm{SNe} \mathrm{Ib}$ and Ic show very similar explosion parameters, i.e. a likely similarity in progenitors.
}

can be detected by iPTF/ZTF. This phase should last at most a few hours, typically less than a day. A cadence of a few hours per field would thus allow the shock breakout cooling tail of such $\mathrm{SNe}$ to be systematically detected. These early observations will be crucial for deriving the progenitor radius with good precision (see, e.g., Bersten et al. 2011, 2013). Our analysis of SDSS-II SNe has demonstrated the importance of observing in several bands in order to constrain the temperature and to construct bolometric light curves that can be compared to theoretical models. iPTF/ZTF have the potential to observe at early times in both the $r$ and $g$ bands and soon will be able to obtain prompt spectra via the SED machine (e.g., Gal-Yam et al. 2014).

After one day, if the ${ }^{56} \mathrm{Ni}$ is not significantly mixed, a plateau phase at relatively low luminosity and with optical temperatures should be observable, lasting about four to ten days, according to several theoretical predictions. A cadence of about a day would be enough to observe at least $\gtrsim 4-10$ epochs on the early plateau. The detection of the dark plateau phase requires that the observations are deep enough to detect luminosities of $\sim 10^{41} \mathrm{erg} \mathrm{s}^{-1}$ (or $M \sim-13 \mathrm{mag}$ ). This means limiting apparent magnitudes of $\sim 22$ mag for objects at distances of $\sim 100 \mathrm{Mpc}$.

For iPTF and ZTF, the best strategy is to increase the cadence rather than the individual exposure times to reach a combined limiting magnitude of $r \sim 23 \mathrm{mag}$, required to discover (or rule out) more dark plateaus of $\mathrm{SNe} \mathrm{Ib} / \mathrm{c}$. The observations of the early plateau, with better constraints on its duration and luminosity, will be crucial to estimate the ${ }^{56} \mathrm{Ni}$ mixing. SN 2006lc is an example of what iPTF/ZTF will systematically observe in the near future, making it possible to precisely disentangle a shallow ${ }^{56} \mathrm{Ni}$ distribution (immediate rise after cooling) from a centrally concentrated ${ }^{56} \mathrm{Ni}$ distribution (delayed rise after a dark plateau).

Finally, after the plateau, the bolometric light curve will rise powered by the ${ }^{56} \mathrm{Ni}$, reaching its peak after $\sim 15-25$ days. To also properly estimate other explosion/progenitor parameters, such as $E_{\mathrm{K}}, M_{\mathrm{ej}}$, it will be necessary for future surveys to follow the early discovered $\mathrm{SNe} \mathrm{Ib} / \mathrm{c}$ until at least a few weeks after peak, to properly fit an analytical or hydrodynamical model and to obtain information on the photospheric velocity evolution from the spectra (see, e.g., Bersten et al. 2013). With our sample we have not explored the late and very late-time epochs (up to 300-400 days after maximum), which would be useful for comparing the late-time light curve slopes (Clocchiatti et al. 1996), thus obtaining information on SN properties, such as the gammaray deposition fraction (Chatzopoulos et al. 2012).

High-cadence spectral coverage with high signal-to-noise ratio data will also allow for distinguishing between helium-rich and helium-poor SNe with high accuracy. As a matter of fact, premaximum spectra obtained soon after discovery may not allow distinguishing between the two groups, since traces of helium can also be present in the early spectra of SN Ic (Branch 2003). In addition, $\mathrm{SNe} \mathrm{IIb}$ and $\mathrm{Ib}$ are impossible to distinguish if there is insufficient spectral coverage (Milisavljevic et al. 2013). In this paper we investigated the differences between SE CC SNe subclasses. Most of our targets were spectroscopically observed before and after maximum, making the classification possible. However, when only limited spectral information was available, the classification was problematic. Future surveys will have to take this into account if they aim to investigate the differences among SE SN subgroups better.

\section{Conclusions}

In this paper we have presented a systematic analysis of the SDSS-II SN Ib/c light curves and an extended comparison 
among the different SE CC subtypes. Although several of the noted trends are not statistically significant for this sample, our work clearly provides valuable hints for future studies of $\mathrm{SN} \mathrm{Ib/c}$ samples with high cadence. The main results of this work are:

- We have enlarged the sample of well-studied SNe Ib/c, analyzing the light curves of 20 events (9 SNe Ib, $6 \mathrm{SNe}$ Ic, 5 SNe Ic-BL ).

- All SNe Ib/c light curves peak first in the bluer and later in the redder optical bands.

- Helium-rich SNe (Ib, IIb) tend to rise to peak in a longer time than helium-poor events (Ic, Ic-BL).

- All the SE SNe subtypes show similar distributions of $\Delta m_{15}$.

- SNe Ic-BL are brighter than Ib and Ic. The last two show different luminosity distributions only if we neglect hostextinction corrections based on the temperature after maximum (SNe Ic then appear brighter and hotter).

- ${ }^{56} \mathrm{Ni}$ mass estimates of $\mathrm{SNe} \mathrm{Ib} / \mathrm{Ic} / \mathrm{Ic}-\mathrm{BL}$ give typical values of $0.3,0.3,1.1 M_{\odot}$, respectively. Energies are on the order of 1.5-1.7 foe for SNe Ib and Ic and about one order of magnitude larger for SNe Ic-BL. Typical ejecta masses for $\mathrm{SNe} \mathrm{Ib} / \mathrm{Ic} / \mathrm{Ic}-\mathrm{BL}$ are 3.6, 5.7, and 5.4 $M_{\odot}$.

- Limits on the early-plateau duration, light curve shape on the rise and early temperature evolution imply strong ${ }^{56} \mathrm{Ni}$ mixing for most SNe Ic and Ic-BL. Therefore, the cause of the spectral difference between SNe Ic and Ib subtypes is not explained well by nickel mixing, but is understood better in the context of a real lack of helium in SN Ic progenitors. We note that the number of SNe Ic is still rather small.

- We detect an early plateau in the case of SN Ib 2006lc and radiation from the shock cooling tail in the early light curves of SNe 2005hm and 2007qx.

- The best limits from PN13 model on the progenitor radii suggest that $\mathrm{SNe} \mathrm{Ib}$, Ic, and Ic-BL arise from compact objects.

- Future surveys will observe numerous young SE SNe with high cadence. We discuss the importance of obtaining multiband photometry and spectra (until a few weeks after peak) in order to constrain the temperature and the photospheric velocity and thus the most important progenitor properties $\left(R,{ }^{56} \mathrm{Ni}\right.$ mixing, $\left.E_{\mathrm{K}}, M_{\mathrm{ej}}\right)$. Deep photometry $(r \sim 23 \mathrm{mag})$ is needed to detect early time plateaus. Good spectral coverage is required to allow distinguishing different SN subclasses.

Acknowledgements. We thank Melina C. Bersten for providing us with the data from her hydrodynamical models, which allowed us to quantify the properties of the early-time plateau as a function of ${ }^{56} \mathrm{Ni}$ mixing, explosion energy, and ejecta mass. The Oskar Klein Centre is funded by the Swedish Research Council. F.T. and M.D.S. acknowledge funding provided by the Instrument Center for Danish Astrophysics (IDA). M.D.S. gratefully acknowledges generous support provided by the Danish Agency for Science and Technology and Innovation realized through a Sapere Aude Level 2 grant. Support for L.G. is provided by the Ministry of Economy, Development, and Tourism's Millennium Science Initiative through grant IC12009, awarded to The Millennium Institute of Astrophysics, MAS. L.G. acknowledges support by CONICYT through FONDECYT grant 3140566. The Dark Cosmology Centre is funded by the Danish National Research Foundation. Funding for the creation and distribution of the SDSS and SDSS-II has been provided by the Alfred P. Sloan Foundation, the Participating Institutions, the National Science Foundation, the US Department of Energy, the National Aeronautics and Space Administration, the Japanese Monbukagakusho, the Max Planck Society, and the Higher Education Funding Council for England. The SDSS Web site is http://www.sdss.org/. The SDSS is managed by the Astrophysical Research Consortium for the Participating Institutions. The Participating Institutions are the American Museum of Natural History, Astrophysical Institute Potsdam, University of Basel, Cambridge University, Case Western Reserve University, University of Chicago, Drexel University, Fermilab, the Institute for Advanced Study, the Japan Participation Group, Johns Hopkins University, the Joint
Institute for Nuclear Astrophysics, the Kavli Institute for Particle Astrophysics and Cosmology, the Korean Scientist Group, the Chinese Academy of Sciences (LAMOST), Los Alamos National Laboratory, the Max-Planck-Institute for Astronomy (MPIA), the Max-Planck-Institute for Astrophysics (MPA), New Mexico State University, Ohio State University, University of Pittsburgh, University of Portsmouth, Princeton University, the United States Naval Observatory, and the University of Washington. The Hobby-Eberly Telescope HET is a joint project of the University of Texas at Austin, the Pennsylvania State University, Stanford University, Ludwig-Maximillians-Universität München, and Georg-August-Universität Göttingen. The HET is named in honor of its principal benefactors, William P. Hobby and Robert E. Eberly. The Subaru Telescope is operated by the National Astronomical Observatory of Japan. The William Herschel Telescope is operated by the Isaac Newton Group, and the Nordic Optical Telescope is operated jointly by Denmark, Finland, Iceland, Norway, and Sweden, both on the island of La Palma in the Spanish Observatorio del Roque de los Muchachos of the Instituto de Astrofisica de Canarias. Observations at the ESO New Technology Telescope at La Silla Observatory were made under program IDs 77.A-0437, 78.A-0325, and 79.A-0715. Kitt Peak National Observatory, National Optical Astronomy Observatory, is operated by the Association of Universities for Research in Astronomy, Inc. (AURA) under cooperative agreement with the National Science Foundation. This research made use of the " $K$-corrections calculator" service available at http://kcor . sai.msu.ru/. This research has made use of the NASA/IPAC Extragalactic Database (NED) which is operated by the Jet Propulsion Laboratory, California Institute of Technology, under contract with the National Aeronautics and Space Administration.

\section{References}

Anderson, J. P., Covarrubias, R. A., James, P. A., Hamuy, M., \& Habergham, S. M. 2010, MNRAS, 407, 2660

Arcavi, I., Gal-Yam, A., Yaron, O., et al. 2011, ApJ, 742, L18

Arnett, W. D. 1982, ApJ, 253, 785

Baltay, C., Bauer, A., Rabinowitz, D., et al. 2007, Central Bureau Electronic Telegrams, 1024, 1

Bazin, G., Ruhlmann-Kleider, V., Palanque-Delabrouille, N., et al. 2011, A\&A, 534, A43

Ben-Ami, S., Gal-Yam, A., Filippenko, A. V., et al. 2012, ApJ, 760, L33

Bersten, M. C., Benvenuto, O., \& Hamuy, M. 2011, ApJ, 729, 61

Bersten, M. C., Benvenuto, O. G., Nomoto, K., et al. 2012, ApJ, 757, 31

Bersten, M. C., Tanaka, M., Tominaga, N., Benvenuto, O. G., \& Nomoto, K. 2013, ApJ, 767, 143

Bersten, M. C., N., Benvenuto, O. G., Folatelli, G., et al. 2014, AJ, 148, 68

Betoule, M., Kessler, R., Guy, J., et al. 2014, A\&A, 568, A22

Bianco, F. B., Modjaz, M., Hicken, M., et al. 2014, ApJS, 213, 19

Blondin, S., Davis, T. M., Krisciunas, K., et al. 2008, ApJ, 682, 724

Branch, D. 2003, A Massive Star Odyssey: From Main Sequence to Supernova,

Proc. of Symp. (San Francisco: ASP), 212, 346

Branch, D., Benetti, S., Kasen, D., et al. 2002, ApJ, 566, 1005

Bufano, F., Pian, E., Sollerman, J., et al. 2012, ApJ, 753, 67

Cano, Z. 2013, MNRAS, 434, 1098

Cano, Z. 2014, ApJ, 794, 121

Cano, Z., \& Jakobsson, P. 2014, MNRAS, submitted [arXiv: 1409. 3570]

Cano, Z., Bersier, D., Guidorzi, C., et al. 2011, ApJ, 740, 41

Cano, Z., Maeda, K., \& Schulze, S. 2014, MNRAS, 438, 2924

Cao, Y., Kasliwal, M. M., Arcavi, I., et al. 2013, ApJ, 775, L7

Cardelli, J. A., Clayton, G. C., \& Mathis, J. S. 1989, ApJ, 345, 245

Chatzopoulos, E., Wheeler, J. C., \& Vinko, J. 2012, ApJ, 746, 121

Chevalier, R. A. 1992, ApJ, 394, 599

Chilingarian, I. V., \& Zolotukhin, I. Y. 2012, MNRAS, 419, 1727

Chilingarian, I. V., Melchior, A.-L., \& Zolotukhin, I. Y. 2010, MNRAS, 405, 1409

Clocchiatti, A., Wheeler, J. C., Benetti, S., \& Frueh, M. 1996, ApJ, 459, 547

Clocchiatti, A., Suntzeff, N. B., Covarrubias, R., \& Candia, P. 2011, AJ, 141, 163

Contardo, G., Leibundgut, B., \& Vacca, W. D. 2000, A\&A, 359, 876

Corsi, A., Ofek, E. O.,Gal-Yam, A., et al. 2012, ApJ, 747, L5

Couch, S. M., Wheeler, J. C., \& Milosavljević, M. 2009, ApJ, 696, 953

Couch, S. M., Pooley, D., Wheeler, J. C., \& Milosavljević, M. 2011, ApJ, 727, 104

Cowen, D. F., Franckowiak, A., \& Kowalski, M. 2010, Astropart. Phys., 33, 19 D’Andrea, C. B., Sako, M., Dilday, B., et al. 2010, ApJ, 708, 661

Dessart, L., Hillier, D. J., Livne, E., et al. 2011, MNRAS, 414, 2985

Dessart, L., Hillier, D. J., Li, C., \& Woosley, S. 2012, MNRAS, 424, 2139

Drout, M. R., Soderberg, A. M., Gal-Yam, A., et al. 2011, ApJ, 741, 97

Elias-Rosa, N., Pastorello, A., Maund, J. R., et al. 2013, MNRAS, 436, L109

Ergon, M., Sollerman, J., Fraser, M., et al. 2014, A\&A, 562, A17 
Ergon, M., Jerkstrand, A., Sollerman, J., et al. 2015, A\&A, accepted [arXiv: 1408.0731]

Ferrero, P., Palazzi, E., Pian, E., \& Savaglio, S. 2007, The Multicolored Landscape of Compact Objects and Their Explosive Origins, AIP Conf. Proc., 924, 120

Filippenko, A. V. 1997, ARA\&A, 35, 309

Filippenko, A. V., Matheson, T., \& Ho, L. C. 1993, ApJ, 415, L103

Folatelli, G., Phillips, M. M., Burns, C. R., et al. 2010, AJ, 139, 120

Folatelli, G., Bersten, M. C., Kuncarayakti, H., et al. 2014, ApJ, 792, 7

Fremling, C., Sollerman, J., Taddia, F., et al. 2014, A\&A, 565, A114

Frieman, J. A., Bassett, B., Becker, A., et al. 2008, AJ, 135, 338

Fukugita, M., Ichikawa, T., Gunn, J. E., et al. 1996, AJ, 111, 1748

Galama, T. J., Vreeswijk, P. M., van Paradijs, J., et al. 1998, Nature, 395, 670

Gal-Yam, A. 2012, Science, 337, 927

Gal-Yam, A., Arcavi, I., Ofek, E. O., et al. 2014, Nature, 509, 471

Goobar, A., Johansson, J., Amanullah, R., et al. 2014, ApJ, 784, L12

Gunn, J. E. Carr, M., Rockosi, C., et al. 1998, AJ, 116, 3040

Gunn, J. E., Siegmund, W. A., Mannery, E. J., et al. 2006, AJ, 131, 2332

Hakobyan, A. A., Adibekyan, V. Z., Aramyan, L. S., et al. 2012, A\&A, 544, A81

Hayden, B. T., Garnavich, P. M., Kessler, R., et al. 2010a, ApJ, 712, 350

Hayden, B. T., Garnavich, P. M., Kasen, D., et al. 2010b, ApJ, 722, 1691

Hjorth, J., Sollerman, J., Møller, P., et al. 2003, Nature, 423, 847

Holtzman, J. A., Marriner, J., Kessler, R., et al. 2008, AJ, 136, 2306

Hunter, D. J., Valenti, S., Kotak, R., et al. 2009, A\&A, 508, 371

Ivezić, Ž., Lupton, R. H., Schlegel, D., et al. 2004, Astron. Nachr., 325, 583

Kessler, R., Becker, A. C., Cinabro, D., et al. 2009, ApJS, 185, 32

Komatsu, E., Dunkley, J., Nolta, M. R., et al. 2009, ApJS, 180, 330

Kulkarni, S. R. 2012 [arXiv: 1202 . 2381]

Kumar, B., Pandey, S. B., Sahu, D. K., et al. 2013, MNRAS, 431, 308

Kuncarayakti, H., Doi, M., Aldering, G., et al. 2013, AJ, 146, 30

Lampeitl, H., Nichol, R. C., Seo, H.-J., et al. 2010, MNRAS, 401, 2331

Leloudas, G., Gallazzi, A., Sollerman, J., et al. 2011, A\&A, 530, A95

Leloudas, G., Chatzopoulos, E., Dilday, B., et al. 2012, A\&A, 541, A129

Li, X., \& Hjorth, J. 2014, A\&A, submitted [arXiv: 1407.3506]

Li, X., Hjorth, J., \& Wojtak, R. 2014, ApJ, submitted [arXiv: 1409. 3567]

Lipkin, Y. M., Ofek, E. O., Gal-Yam, A., et al. 2004, ApJ, 606, 381

Lyman, J., Bersier, D., James, P., et al. 2014, MNRAS, submitted [arXiv: 1406.3667]

Maeda, K., Kawabata, K., Mazzali, P. A., et al. 2008, Science, 319, 1220

Malesani, D., Fynbo, J. P. U., Hjorth, J., et al. 2009, ApJ, 692, L84

Massey, P. 2003, ARA\&A, 41, 15

Matheson, T., Filippenko, A. V., Li, W., Leonard, D. C., \& Shields, J. C. 2001, AJ, 121, 1648

Maund, J. R., Smartt, S. J., Kudritzki, R. P., Podsiadlowski, P., \& Gilmore, G. F. 2004, Nature, 427, 129

Maund, J. R., Wheeler, J. C., Patat, F., et al. 2007a, A\&A, 475, L1

Maund, J. R., Wheeler, J. C., Patat, F., et al. 2007b, ApJ, 671, 1944

Maund, J. R., Wheeler, J. C., Baade, D., et al. 2009, ApJ, 705, 1139
Maund, J. R., Fraser, M., Ergon, M., et al. 2011, ApJ, 739, L37

Mazzali, P. A., Valenti, S., Della Valle, M., et al. 2008, Science, 321, 1185

Milisavljevic, D., Margutti, R., Soderberg, A. M., et al. 2013, ApJ, 767, 71

Modjaz, M., Li, W., Butler, N., et al. 2009, ApJ, 702, 226

Modjaz, M., Kewley, L., Bloom, J. S., et al. 2011, ApJ, 731, L4

Modjaz, M., Blondin, S., Kirshner, R. P., et al. 2014, AJ, 147, 99

Morrell, N., Folatelli, G., \& Phillips, M. M. 2005, IAU Circ., 8611, 2

Mosher, J., Sako, M., Corlies, L., et al. 2012, AJ, 144, 17

Nakar, E., \& Piro, A. L. 2014, ApJ, 788, 193

Nakar, E., \& Sari, R. 2010, ApJ, 725, 904

Nugent, P. E., Sullivan, M., Cenko, S. B., et al. 2011, Nature, 480, 344

Pastorello, A., Kasliwal, M. M., Crockett, R. M., et al. 2008, MNRAS, 389, 955

Phillips, M. M. 1993, ApJ, 413, L105

Pignata, G., Stritzinger, M., Soderberg, A., et al. 2011, ApJ, 728, 14

Piro, A. L., \& Nakar, E. 2013, ApJ, 769, 67

Poznanski, D., Ganeshalingam, M., Silverman, J. M., \& Filippenko, A. V. 2011, MNRAS, 415, L81

Poznanski, D., Prochaska, J. X., \& Bloom, J. S. 2012, MNRAS, 426, 1465

Richardson, D., Branch, D., \& Baron, E. 2006, AJ, 131, 2233

Richardson, D., Jenkins, R. L., III, Wright, J., \& Maddox, L. 2014, AJ, 147, 118

Richmond, M. W., Treffers, R. R., Filippenko, A. V., et al. 1994, AJ, 107, 1022

Richmond, M. W., van Dyk, S. D., Ho, W., et al. 1996, AJ, 111, 327

Roy, R., Kumar, B., Maund, J. R., et al. 2013, MNRAS, 434, 2032

Sako, M., Bassett, B., Becker, A., et al. 2008, AJ, 135, 348

Sako, M., Bassett, B., Becker, A. C., et al. 2014, ApJS, submitted [arXiv: 1401.3317]

Sanders, N. E., Soderberg, A. M., Levesque, E. M., et al. 2012, ApJ, 758, 132

Schlafly, E. F., \& Finkbeiner, D. P. 2011, ApJ, 737, 103

Smith, N., Li, W., Filippenko, A. V., \& Chornock, R. 2011, MNRAS, 412, 1522

Soderberg, A. M., Berger, E., Page, K. L., et al. 2008, Nature, 453, 469

Sollerman, J., Jaunsen, A. O., Fynbo, J. P. U., et al. 2006, A\&A, 454, 503

Sollerman, J., Mörtsell, E., Davis, T. M., et al. 2009, ApJ, 703, 1374

Stritzinger, M., Hamuy, M., Suntzeff, N. B., et al. 2002, AJ, 124, 2100

Stritzinger, M., Mazzali, P., Phillips, M. M., et al. 2009, ApJ, 696, 713

Takaki, K., Kawabata, K. S., Yamanaka, M., et al. 2013, ApJ, 772, L17

Taubenberger, S., Pastorello, A., Mazzali, P. A., et al. 2006, MNRAS, 371, 1459

Taubenberger, S., Navasardyan, H., Maurer, J. I., et al. 2011, MNRAS, 413, 2140

Valenti, S., Benetti, S., Cappellaro, E., et al. 2008, MNRAS, 383, 1485

Valenti, S., Fraser, M., Benetti, S., et al. 2011, MNRAS, 416, 3138

Valenti, S., Taubenberger, S., Pastorello, A., et al. 2012, ApJ, 749, L28

Wheeler, J. C., Harkness, R. P., Barker, E. S., Cochran, A. L., \& Wills, D. 1987, ApJ, 313, L69

Wright, E. L. 2006, PASP, 118, 1711

Yoon, S.-C., Woosley, S. E., \& Langer, N. 2010, ApJ, 725, 940

York, D. G., Adelman, J., Anderson, J. E., Jr., et al. 2000, AJ, 120, 1579

Zheng, C., Romani, R. W., Sako, M., et al. 2008, AJ, 135, 1766

Zheng, W., Shivvers, I., Filippenko, A. V., et al. 2014, ApJ, 783, L24 

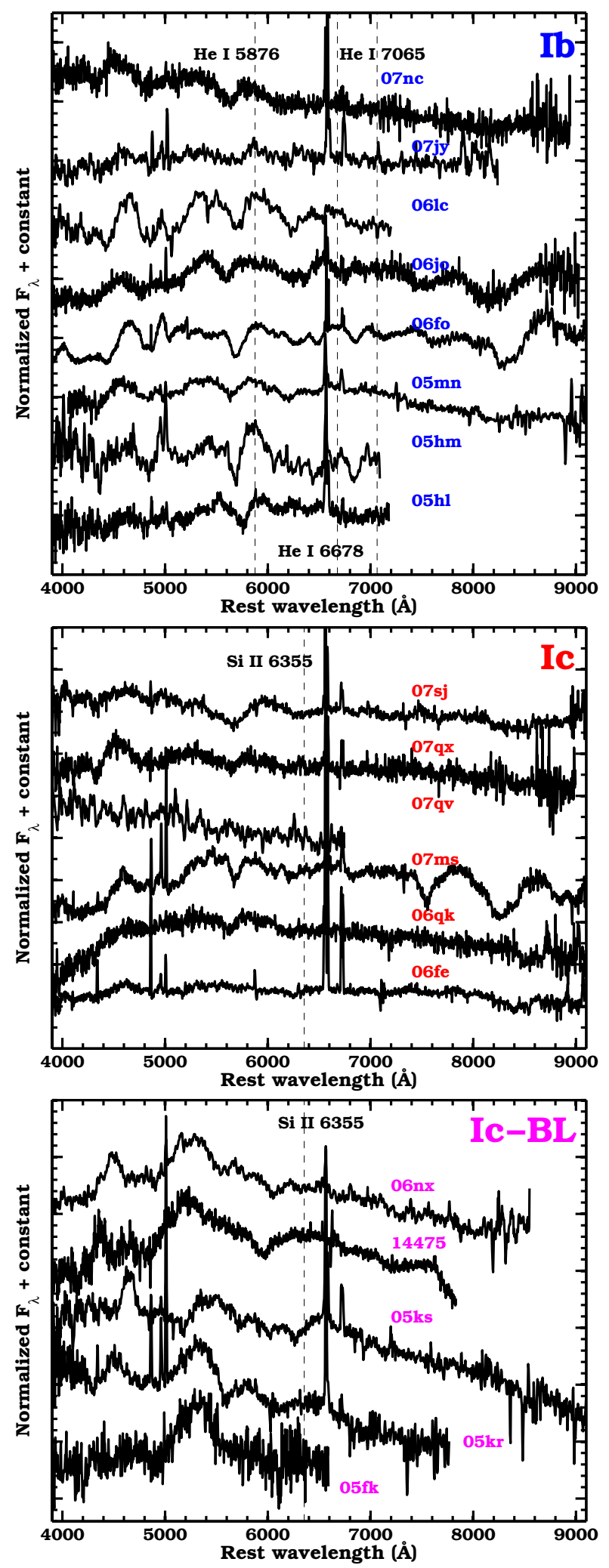

Fig. 1. Selected spectra (data from Sako et al. 2014) for 19 SDSS SNe Ib/c (SN 2007gl was classified by Baltay et al. 2007). The vertical dashed lines indicate the rest wavelengths of He I $\lambda \lambda 5876,6678,7065$, and Si II $\lambda 6355$ at zero velocity. The epoch of each spectrum is marked in Fig. 4 with a vertical red segment. Most of the spectra show narrow emission lines from the host galaxy. 

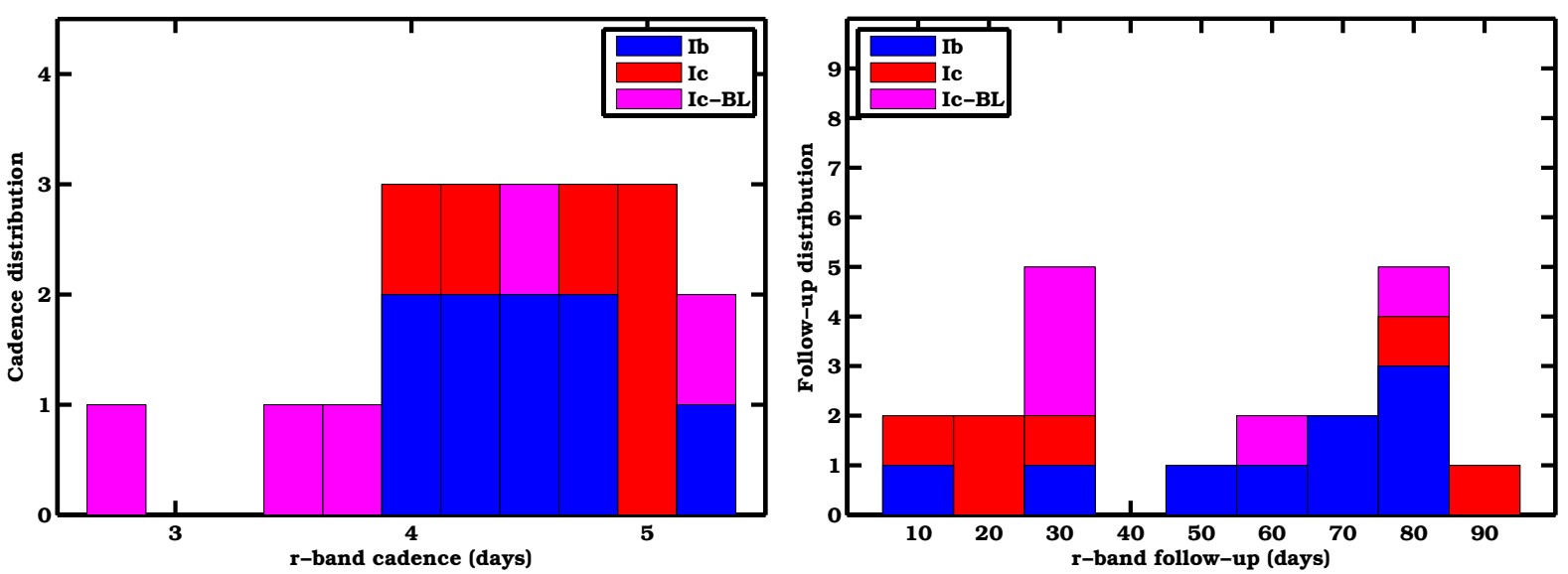

Fig. 7. Lefthand panel: $r$-band cadence distribution for the SDSS SN Ib/c sample. Typical values are between 4 and 5 days. Righthand panel: $r$-band follow-up distribution for the SDSS SN Ib/c sample. SNe were followed for a minimum of $\sim 10$ days to a maximum of $\sim 90$ days after discovery.

Table 1. SDSS sample of $20 \mathrm{SNe} \mathrm{Ib}$, Ic and Ic-BL.

\begin{tabular}{|c|c|c|c|c|c|c|c|c|c|}
\hline $\mathrm{SN}$ & $\begin{array}{l}\text { RA (J2000) } \\
\text { (hh:mm:ss) }\end{array}$ & $\begin{array}{c}\text { Dec (J2000) } \\
\text { (dd:mm:ss) }\end{array}$ & Type & Redshift & $\begin{array}{r}\text { Distance } \\
(\mathrm{Mpc})\end{array}$ & Host galaxy & $\begin{array}{c}E(B-V)_{\mathrm{MW}} \\
(\mathrm{mag})\end{array}$ & $\begin{array}{c}E(B-V)_{\text {host }}{ }^{*} \\
\text { (mag) }\end{array}$ & $\begin{array}{l}M_{g}^{\mathrm{gal}{ }_{* *}} \\
(\mathrm{mag})\end{array}$ \\
\hline $2005 \mathrm{fk}$ & $21: 15: 19.84$ & $-00: 22: 58.6$ & Ic-BL & 0.264 & 1341.1 & A211519-0022 & 0.054 & & -19.90 \\
\hline $2005 \mathrm{hl}$ & $20: 55: 19.79$ & $+00: 32: 34.7$ & $\mathrm{Ib}$ & 0.023 & 100.4 & A205519+0032 & 0.073 & 0.533 & -20.23 \\
\hline $2005 \mathrm{hm}$ & $21: 39: 00.65$ & $-01: 01: 38.7$ & $\mathrm{Ib}$ & 0.035 & 151.6 & A213900-0101 & 0.048 & 0 & -15.51 \\
\hline $2005 \mathrm{kr}$ & $03: 08: 29.66$ & $+00: 53: 20.2$ & Ic-BL & 0.134 & 627.1 & A $030829+0053$ & 0.087 & 0.075 & -17.57 \\
\hline $2005 \mathrm{ks}$ & $21: 37: 56.56$ & $-00: 01: 56.9$ & Ic-BL & 0.099 & 451.5 & A213756-0001 & 0.050 & 0.537 & -19.39 \\
\hline $2005 \mathrm{mn}$ & $03: 49: 18.44$ & $-00: 41: 31.4$ & $\mathrm{Ib}$ & 0.047 & 209.2 & A034918-0041 & 0.166 & & -18.86 \\
\hline 2006fe & $20: 52: 09.10$ & $-00: 30: 39.3$ & Ic & 0.070 & 316.3 & SDSS J205209.10-003039.2 & 0.098 & 0.132 & -20.56 \\
\hline 2006 fo & $02: 32: 38.89$ & $+00: 37: 03.0$ & $\mathrm{Ib}$ & 0.021 & 89.4 & UGC 2019 & 0.026 & 0.301 & -20.31 \\
\hline 14475 & $22: 24: 30.90$ & $+00: 12: 12.3$ & Ic-BL & 0.149 & 705.3 & SDSS J222430.86+001212.3 & 0.072 & 0.650 & -18.05 \\
\hline 2006jo & $01: 23: 14.72$ & $-00: 19: 46.7$ & $\mathrm{Ib}$ & 0.077 & 345.8 & A012314-0019 & 0.032 & 0.460 & -20.81 \\
\hline $2006 \mathrm{lc}$ & $22: 44: 24.48$ & $-00: 09: 53.5$ & $\mathrm{Ib}$ & 0.016 & 69.7 & NGC7364 & 0.057 & 0.510 & -21.20 \\
\hline $2006 n x$ & $03: 33: 30.63$ & $-00: 40: 38.2$ & Ic-BL & 0.137 & 641.9 & A033330-0040 & 0.108 & 0.461 & -19.19 \\
\hline $2006 \mathrm{qk}$ & $22: 25: 32.38$ & $+00: 09: 15.1$ & Ic & 0.058 & 259.5 & A222532+0009 & 0.075 & & -17.96 \\
\hline $2007 \mathrm{gl}$ & $03: 11: 33.21$ & $-00: 44: 46.7$ & $\mathrm{Ib}$ & 0.028 & 122.6 & KUG 0309-009 & 0.059 & & -19.51 \\
\hline 2007jy & $20: 51: 21.43$ & $+00: 23: 57.8$ & $\mathrm{Ib}$ & 0.180 & 869.0 & A205121+0023 & 0.095 & & -19.86 \\
\hline $2007 \mathrm{~ms}$ & $20: 32: 18.34$ & $-01: 00: 53.1$ & Ic & 0.039 & 170.9 & A203218-0100 & 0.184 & 0.040 & -17.76 \\
\hline $2007 \mathrm{nc}$ & $00: 01: 09.30$ & $+01: 04: 06.5$ & $\mathrm{Ib}$ & 0.087 & 393.9 & A000109+0104 & 0.025 & 0.227 & -20.21 \\
\hline $2007 q v$ & $22: 35: 07.91$ & $-01: 06: 37.5$ & Ic & 0.095 & 433.5 & A223507-0106 & 0.048 & 0 & -19.61 \\
\hline 2007qx & $00: 27: 41.78$ & $+01: 13: 59.7$ & Ic & 0.080 & 363.3 & A $002741+0113$ & 0.023 & 0.371 & -20.20 \\
\hline 2007sj & $00: 10: 39.63$ & $-00: 03: 10.2$ & Ic & 0.039 & 170.1 & A001039-0003 & 0.032 & $\ldots$ & -21.24 \\
\hline
\end{tabular}

Notes. Typical errors on the distance are on the order of 5-10\%. Redshifts are usually known with a precision down to $\delta z \sim 0.001$ (Zheng et al. 2008). ${ }^{(*)}$ See Sect. 3.3.1 for details. ${ }^{(*)}$ The absolute magnitude in $g$ band for each host galaxy is corrected for the Milky Way extinction at the position of the host center and K-corrected. 
Table 2. Spectral log for the SDSS SNe Ib/c.

\begin{tabular}{|c|c|c|c|c|c|c|c|c|c|}
\hline SN & Type & $\begin{array}{l}\text { Spectral epoch } \\
\text { (MJD-53 000) }\end{array}$ & Telescope & $\begin{array}{l}\text { Velocity } \\
\left(\mathrm{km} \mathrm{s}^{-1}\right)\end{array}$ & $\mathrm{SN}$ & Type & $\begin{array}{l}\text { Spectral epoch } \\
\text { (MJD-53 000) }\end{array}$ & Telescope & $\begin{array}{l}\text { Velocity } \\
\left(\mathrm{km} \mathrm{s}^{-1}\right)\end{array}$ \\
\hline $2005 \mathrm{fk}$ & Ic-BL & $640.10 *$ & HET & $11700(\mathrm{Si})$ & $2006 \mathrm{lc}$ & $\overline{\mathrm{Ib}}$ & 1030.17 & $\overline{\mathrm{H}}$ & $6080(\mathrm{Si})$ \\
\hline $2005 \mathrm{hl}$ & $\mathrm{Ib}$ & $665.12 *$ & MGH & $5450(\mathrm{He})$ & & & 1035.19 & $\mathrm{H}$ & $11261(\mathrm{He})$ \\
\hline $2005 \mathrm{hm}$ & $\mathrm{Ib}$ & $663.19 *$ & MGH & $9470(\mathrm{He})$ & & & $1043.14 *$ & $\mathrm{H}$ & $9050(\mathrm{He})$ \\
\hline $2005 \mathrm{kr}$ & Ic-BL & $696.19 *$ & HET & $12200(\mathrm{Si})$ & & & 1059.13 & $\mathrm{H}$ & $8246(\mathrm{He})$ \\
\hline $2005 \mathrm{ks}$ & Ic-BL & 696.06* & HET & $15500(\mathrm{Si})$ & & & 1082.08 & ESO-NTT & $7141(\mathrm{He})$ \\
\hline \multirow[t]{6}{*}{$2005 \mathrm{mn}$} & $\mathrm{Ib}$ & $705.29 *$ & HET & $11900(\mathrm{He})$ & & & 1069.06 & $\mathrm{H}$ & \\
\hline & & 708.35 & ARC & & & & 1072.61 & $\mathrm{H}$ & \\
\hline & & 711.27 & HET & $11000(\mathrm{He})$ & $2006 n x$ & Ic-BL & 1056.19 & NOT & $15400(\mathrm{Si})$ \\
\hline & & 767.13 & HET & & & & 1057.33 & $\mathrm{KP}$ & \\
\hline & & 767.13 & HET & & & & 1058.36 & $\mathrm{H}$ & \\
\hline & & 767.13 & HET & & & & $1063.31 *$ & ESO-NTT & $14190(\mathrm{Si})$ \\
\hline \multirow[t]{2}{*}{$2006 f e$} & Ic & $994.02 *$ & ESO-NTT & $4760(\mathrm{Si})$ & & & 1064.32 & ESO-NTT & $13190(\mathrm{Si})$ \\
\hline & & 995.26 & SUBARU & $5340(\mathrm{Si})$ & 2006qk & Ic & $1065.11 *$ & ESO-NTT & $13740(\mathrm{He})$ \\
\hline \multirow[t]{11}{*}{ 2006fo } & $\mathrm{Ib}$ & 995.16 & ESO-NTT & $10480(\mathrm{He})$ & & & 1072.06 & $\mathrm{H}$ & \\
\hline & & 1004.40 & KP & $10080(\mathrm{He})$ & & & 1073.07 & $\mathrm{H}$ & \\
\hline & & $1010.44 *$ & ARC & $9530(\mathrm{He})$ & & & 1085.04 & ESO-NTT & \\
\hline & & 1015.38 & $\mathrm{H}$ & $9280(\mathrm{He})$ & 2007jy & $\mathrm{Ib}$ & $1363.09 *$ & ESO-NTT & \\
\hline & & 1018.41 & $\mathrm{H}$ & $9380(\mathrm{He})$ & $2007 \mathrm{~ms}$ & Ic & 1361.03 & ESO-NTT & $11410(\mathrm{He})$ \\
\hline & & 1024.36 & ESO-NTT & $9030(\mathrm{He})$ & & & $1387.05 *$ & ESO-NTT & $7130(\mathrm{Si})$ \\
\hline & & 1027.36 & $\mathrm{H}$ & $8680(\mathrm{He})$ & & & 1389.04 & ESO-NTT & \\
\hline & & 1031.31 & $\mathrm{H}$ & $8430(\mathrm{He})$ & $2007 \mathrm{nc}$ & $\mathrm{Ib}$ & $1390.16^{*}$ & ESO-NTT & $12680(\mathrm{Si})$ \\
\hline & & 1037.32 & $\mathrm{H}$ & $8030(\mathrm{He})$ & $2007 \mathrm{qv}$ & Ic & $1413.10 *$ & $\mathrm{H}$ & \\
\hline & & 1059.33 & $\mathrm{H}$ & & & & 1415.09 & $\mathrm{H}$ & \\
\hline & & 1064.27 & ESO-NTT & $7380(\mathrm{He})$ & $2007 q x$ & Ic & $1417.18 *$ & ESO-NTT & $11790(\mathrm{He})$ \\
\hline 14475 & Ic-BL & $1023.31 *$ & SUBARU & $18700(\mathrm{Si})$ & $2007 \mathrm{sj}$ & Ic & 1429.13 & $\mathrm{H}$ & \\
\hline \multirow[t]{2}{*}{ 2006jo } & $\mathrm{Ib}$ & 1019.30 & & & & & $1441.14 *$ & ESO-NTT & \\
\hline & & $1023.26^{*}$ & ESO-NTT & $14360(\mathrm{He})$ & & & & & \\
\hline
\end{tabular}

Notes. All the spectra were released by Sako et al. (2014). ARC = Astrophysical Research Consortium $3.5 \mathrm{~m}$ telescope at the Apache Point Observatory (New Mexico), ESO-NTT = New Technology $3.6 \mathrm{~m}$ Telescope in La Silla (Chile), HET = Hobby-Eberly $9.2 \mathrm{~m}$ Telescope at McDonald Observatory (Texas), $\mathrm{H}=$ Hiltner $2.4 \mathrm{~m}$ telescope at the Michigan-Dartmouth-MIT observatory (Arizona), KP = Kitt Peak National Observatory Mayall $4 \mathrm{~m}$ telescope (Arizona), MGH = McGraw-Hill $1.3 \mathrm{~m}$ telescope at the Michigan-Dartmouth-MIT observatory (Arizona), NOT $=$ Nordic Optical $2.5 \mathrm{~m}$ Telescope in La Palma (Spain), SUBARU $=8.2 \mathrm{~m}$ Subaru Telescope at the National Astronomical Observatory of Japan (Hawaii). (He): Velocity measured from He I 25876 . (Si): Velocity measured from Si II $\lambda 6355 .{ }^{(*)}$ Spectra plotted in Fig. 1. 


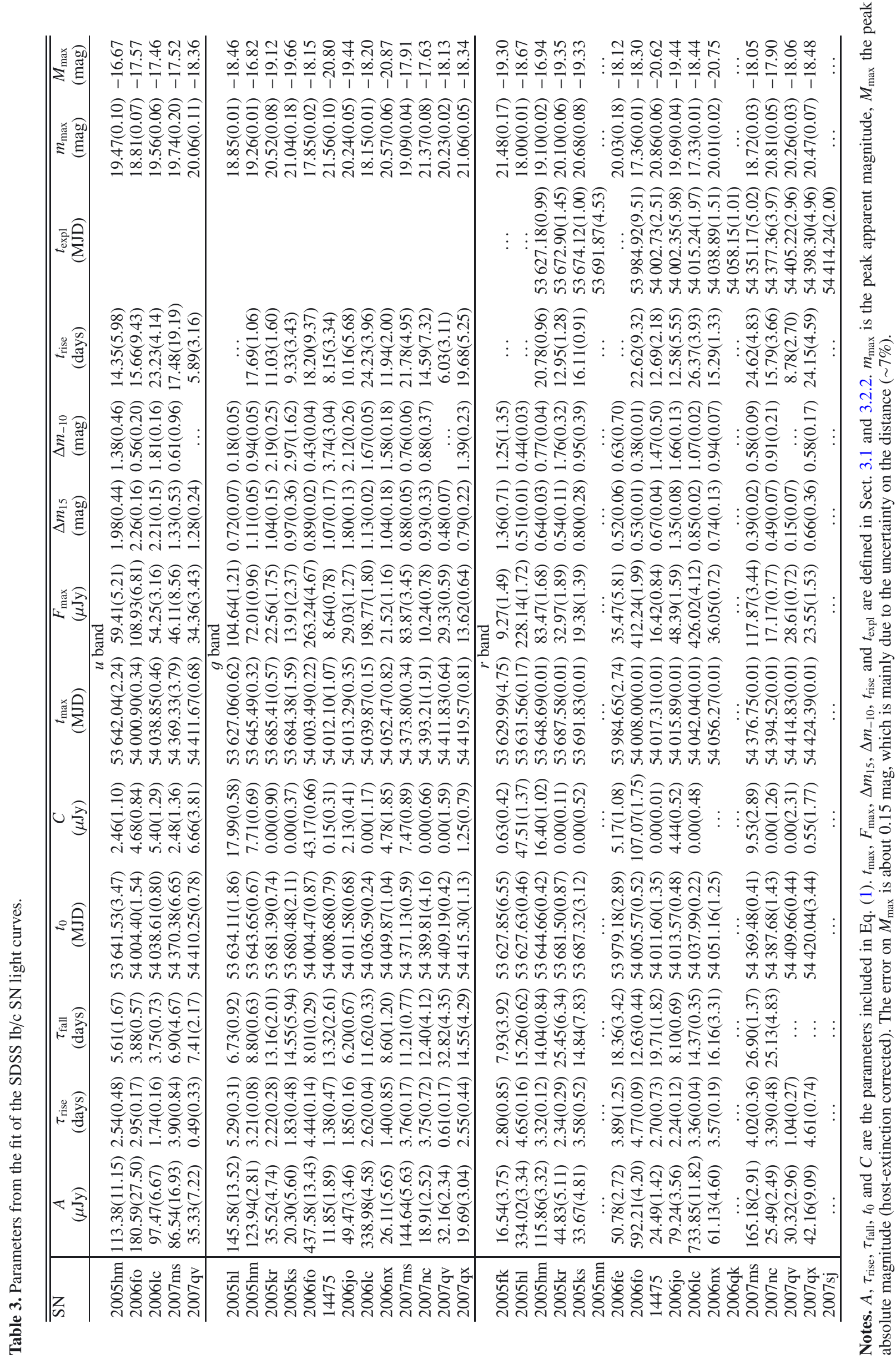




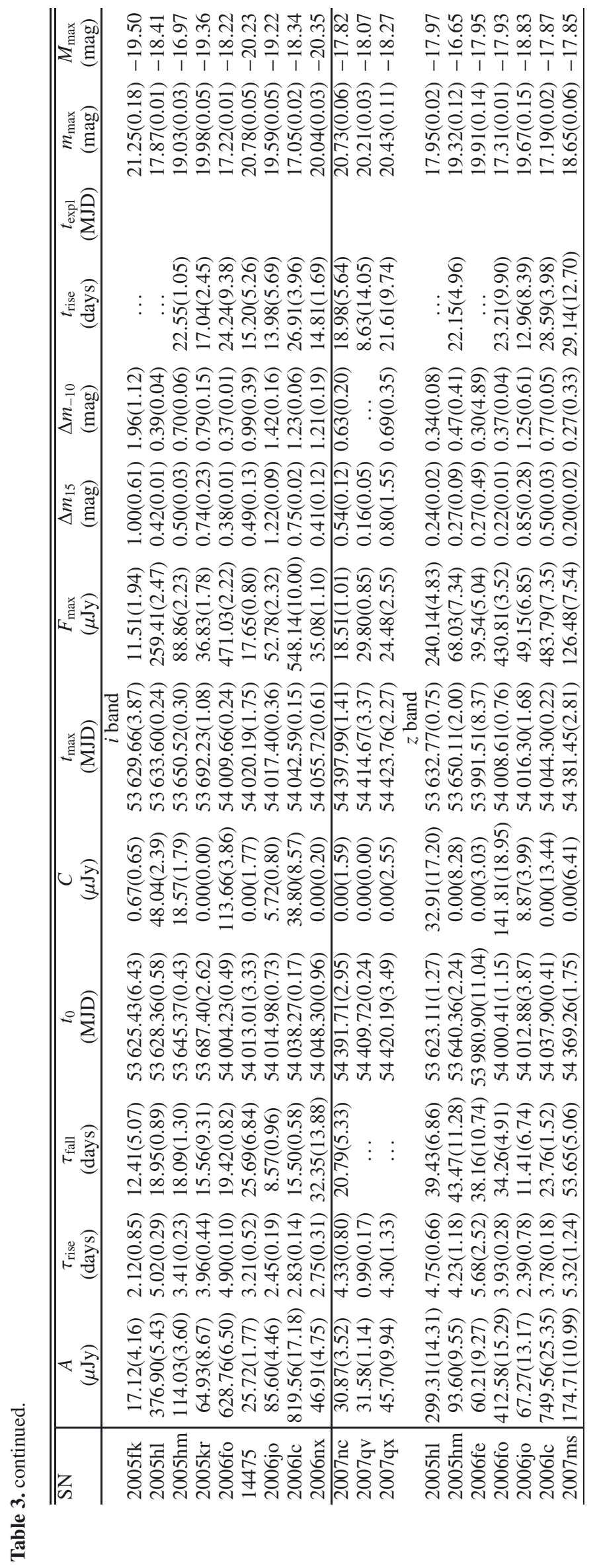


F. Taddia et al.: Early-time light curves of SDSS-II SNe Ib/c

Table 4. Well-constrained $R / r$-band light-curve rise-times and $\Delta m_{-10}$ for 4, 13, 10 and $9 \mathrm{SNe} \mathrm{IIb}$, Ib, Ic and Ic-BL in the literature.

\begin{tabular}{|c|c|c|c|c|}
\hline SN & Type & $\begin{array}{c}t_{\text {rise }} \\
\text { (days) }\end{array}$ & $\begin{array}{l}\Delta m_{-10} \\
(\mathrm{mag})\end{array}$ & Ref. \\
\hline 1993J & IIlb & $22.53(3.24)$ & $0.77(0.15)$ & Kumar et al. (2013), Richmond et al. (1994) \\
\hline 2008ax & $\mathrm{IIb}$ & $21.50(0.40)$ & $0.63(0.15)$ & Taubenberger et al. (2011) \\
\hline 2011dh & $\mathrm{IIb}$ & $21.86(0.23)$ & $0.61(0.10)$ & Ergon et al. (2014) \\
\hline $2011 \mathrm{fu}$ & $\mathrm{IIb}$ & $26.40(2.90)$ & $0.42(0.15)$ & Kumar et al. (2013) \\
\hline $1998 \mathrm{dt}$ & $\mathrm{Ib}$ & $17.60(3.00)$ & $1.00(0.10)$ & Matheson et al. (2001) \\
\hline 1999ex & $\mathrm{Ib}$ & $20.49(0.52)$ & $0.38(0.10)$ & Stritzinger et al. (2002); \\
\hline $2004 \mathrm{dk}$ & $\mathrm{Ib}$ & $23.75(1.55)$ & $0.50(0.10)$ & Drout et al. (2011) \\
\hline $2005 \mathrm{hm}$ & $\mathrm{Ib}$ & 21.06(1.17) & $0.76(0.03)$ & This paper \\
\hline 2006fo & $\mathrm{Ib}$ & & $0.38(0.01)$ & This paper \\
\hline 2006lc & $\mathrm{Ib}$ & 26.41(5.18) & $1.08(0.02)$ & This paper \\
\hline $2007 Y$ & $\mathrm{Ib}$ & $21.00(0.50)$ & $0.67(0.10)$ & Stritzinger et al. (2009) \\
\hline 2007nc & $\mathrm{Ib}$ & & $0.80(0.15)$ & This paper \\
\hline 2007uy & $\mathrm{Ib}$ & $21.72(2.50)$ & & Roy et al. (2013), explosion date from modelling of radio data \\
\hline 2008D & $\mathrm{Ib}$ & 20.75(1.00) & $0.31(0.10)$ & Soderberg et al. (2008), Malesani et al. (2009) \\
\hline 2009jf & $\mathrm{Ib}$ & $24.50(1.00)$ & $0.34(0.10)$ & Valenti et al. (2011), good pre-explosion limit \\
\hline $2011 \mathrm{ei}$ & $\mathrm{Ib}$ & $19.50(2.50)$ & $0.69(0.20)$ & Milisavljevic et al. (2013) \\
\hline iPTF 13bvn & $\mathrm{Ib}$ & $18.55(0.70)$ & $0.57(0.10)$ & Fremling et al. (2014) \\
\hline 1994I & Ic & $10.01(1.02)$ & $2.50(0.50)$ & Richmond et al. (1996) \\
\hline 2004aw & Ic & $\ldots$ & $0.27(0.05)$ & Taubenberger et al. (2006) \\
\hline 2004dn & Ic & $\ldots$ & $0.60(0.15)$ & Drout et al. (2011) \\
\hline 2004fe & Ic & $\ldots$ & $1.20(0.10)$ & Drout et al. (2011) \\
\hline $2007 \mathrm{gr}$ & Ic & $15.50(3.02)$ & $0.73(0.04)$ & Hunter et al. (2009) \\
\hline $2007 \mathrm{~ms}$ & Ic & & $0.56(0.08)$ & This paper \\
\hline 2007qv & Ic & $8.72(3.08)$ & & This paper \\
\hline 2007qx & Ic & $\ldots$ & $0.62(0.17)$ & This paper \\
\hline PTF 10vgv & Ic & $11.91(0.85)$ & $2.63(0.50)$ & Corsi et al. (2012) \\
\hline $2013 \mathrm{dk}$ & Ic & & $1.33(0.20)$ & Elias-Rosa et al. (2013), $\Delta m_{-10}$ scaled to $r$ from $V$ \\
\hline 1998bw & Ic-BL & $17.50(0.50)$ & $0.68(0.15)$ & Clocchiatti et al. (2011) \\
\hline 2003jd & Ic-BL & $16.20(1.00)$ & $\ldots$ & Valenti et al. (2008), only error on max epoch \\
\hline $2005 \mathrm{kr}$ & Ic-BL & $15.40(1.45)$ & $1.18(0.16)$ & This paper \\
\hline 14475 & Ic-BL & $14.36(2.58)$ & $1.15(0.31)$ & This paper \\
\hline 2006аj & Ic-BL & $12.30(0.50)$ & $2.40(0.50)$ & Ferrero et al. (2007), $\Delta m_{-10}$ is extrapolated \\
\hline $2006 n x$ & Ic-BL & $14.99(1.41)$ & $1.10(0.12)$ & This paper \\
\hline 2009bb & Ic-BL & $14.65(1.25)$ & $1.37(0.20)$ & Pignata et al. (2011) \\
\hline 2010bh & Ic-BL & $8.00(1.00)$ & $2.00(0.50)$ & Bufano et al. (2012), $\Delta m_{-8}$ \\
\hline PTF 12gzk & Ic-BL & 20.74(0.62) & $0.45(0.10)$ & Ben-Ami et al. (2012) \\
\hline
\end{tabular}




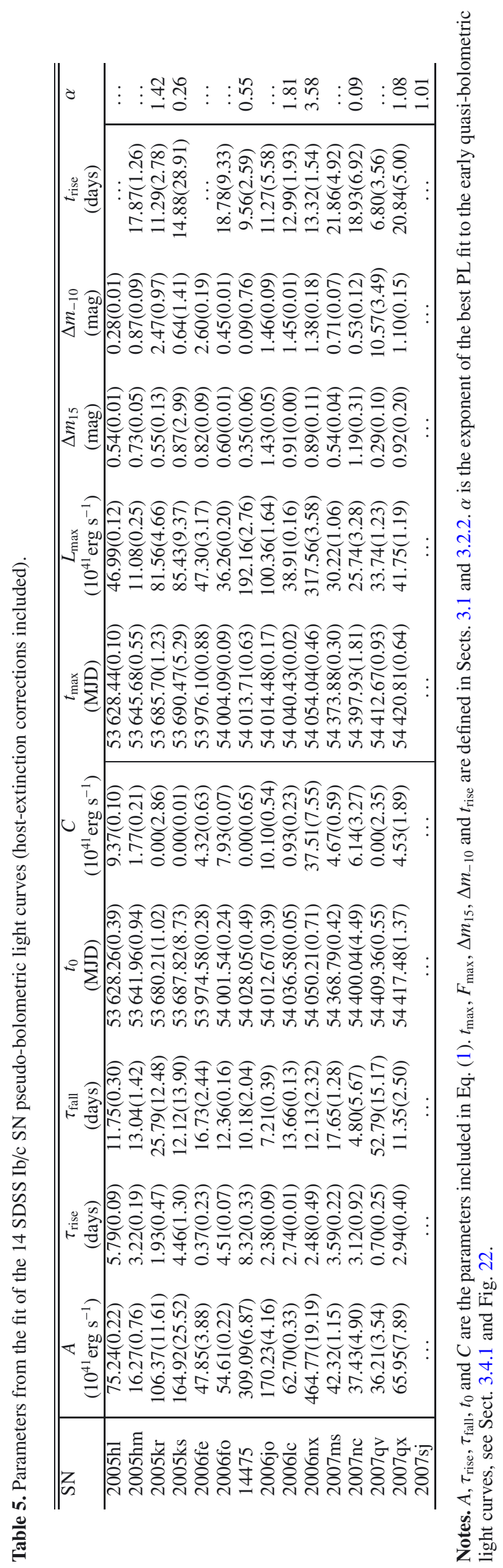


F. Taddia et al.: Early-time light curves of SDSS-II SNe Ib/c

Table 6. ${ }^{56} \mathrm{Ni}$ mass, ejecta mass, explosion energy and progenitor radius for the SDSS sample of SNe Ib/c.

\begin{tabular}{|c|c|c|c|c|c|}
\hline SN & Type & $\begin{array}{l}M_{56} \mathrm{Ni} \\
\left(M_{\odot}\right)\end{array}$ & $\begin{array}{c}M_{\mathrm{ej}} \\
\left(M_{\odot}\right)\end{array}$ & $\begin{array}{c}E_{\mathrm{K}} \\
\text { (foe) }\end{array}$ & $\begin{array}{l}R \\
\left(R_{\odot}\right)\end{array}$ \\
\hline $2005 \mathrm{fk}$ & Ic-BL & & & & $<38.1$ \\
\hline $2005 \mathrm{hl}$ & $\mathrm{Ib}$ & $0.33_{-0.05}^{+0.05}$ & $1.56_{-1.05}^{+2.88}$ & $0.64_{-0.43}^{+2.40}$ & $<357.4$ \\
\hline $2005 \mathrm{hm}$ & $\mathrm{Ib}$ & $0.11_{-0.01}^{+0.01}$ & $3.45_{-0.40}^{+1.54}$ & $0.36_{-0.04}^{+0.50}$ & $<57.2$ \\
\hline $2005 \mathrm{kr}$ & Ic-BL & $0.71_{-0.04}^{+0.04}$ & $7.75_{-1.94}^{+5.48}$ & $15.18_{-3.80}^{+28.00}$ & $<6.0$ \\
\hline 2005ks & Ic-BL & $0.60_{-0.02}^{+0.02}$ & $3.39_{-0.32}^{+1.53}$ & $1.17_{-0.11}^{+1.65}$ & $<87.9$ \\
\hline $2005 \mathrm{mn}$ & Ib & $\ldots$ & $\ldots$ & $\ldots$ & $<25.4$ \\
\hline $2006 \mathrm{fe}$ & Ic & $\ldots$ & $\ldots$ & $\ldots$ & $<116.0$ \\
\hline 2006fo & $\mathrm{Ib}$ & $0.38_{-0.06}^{+0.01}$ & $6.04_{-2.73}^{+5.57}$ & $1.90_{-0.86}^{+1.65}$ & $<172.2$ \\
\hline 14475 & Ic-BL & $1.27_{-0.09}^{+0.08}$ & $2.90_{-1.48}^{+3.38}$ & $4.71_{-2.41}^{+12.29}$ & $<93.1$ \\
\hline 2006jo & $\mathrm{Ib}$ & $0.42_{-0.08}^{+0.09}$ & $2.51_{-2.14}^{+4.98}$ & $2.83_{-2.42}^{+11.24}$ & $<152.1$ \\
\hline 2006lc & $\mathrm{Ib}$ & $0.30_{-0.03}^{+0.03}$ & $3.67_{-1.79}^{+4.96}$ & $1.60_{-0.78}^{+4.68}$ & 1.7 \\
\hline $2006 n x$ & Ic-BL & $1.86_{-0.12}^{+0.12}$ & $7.52_{-1.74}^{+4.83}$ & $21.60_{-5.01}^{+37.53}$ & $<53.9$ \\
\hline 2006qk & Ic & $\ldots$ & $\ldots$ & $\ldots$ & $<5.1$ \\
\hline $2007 \mathrm{~ms}$ & Ic & $0.39_{-0.03}^{+0.03}$ & $9.12_{-2.76}^{+8.45}$ & $2.05_{-0.62}^{+4.54}$ & $<40.7$ \\
\hline $2007 \mathrm{nc}$ & $\mathrm{Ib}$ & $0.24_{-0.03}^{+0.02}$ & $4.38_{-2.02}^{+4.87}$ & $1.54_{-0.71}^{+3.88}$ & $<34.4$ \\
\hline $2007 q v$ & Ic & $0.20_{-0.03}^{+0.04}$ & $1.91_{-1.34}^{+3.81}$ & $1.92_{-1.34}^{+7.64}$ & 11.5 \\
\hline $2007 q x$ & Ic & $0.40_{-0.08}^{+0.15}$ & $6.21_{-3.20}^{+12.10}$ & $1.28_{-0.66}^{+5.02}$ & $<28.7$ \\
\hline 2007sj & Ic & $\ldots$ & $\ldots$ & $\ldots$ & $<1.6$ \\
\hline$\langle\mathrm{Ib}\rangle$ & & $0.30 \pm 0.05$ & $3.60 \pm 0.63$ & $1.48 \pm 0.36$ & \\
\hline$\langle\mathrm{Ic}\rangle$ & & $0.33 \pm 0.07$ & $5.75 \pm 2.09$ & $1.75 \pm 0.24$ & \\
\hline$\langle\mathrm{Ic}-\mathrm{BL}\rangle$ & & $1.11 \pm 0.29$ & $5.39 \pm 1.30$ & $10.66 \pm 4.70$ & \\
\hline
\end{tabular}

Notes. The reported errors are due to the uncertainty on the rise time. Typical uncertainties on the ${ }^{56} \mathrm{Ni}$ mass due to the error on the distance are $\sim 7 \%$.

Table 7. Limits on the early plateau parameters and peak luminosity values for the SDSS sample of SNe Ib/c (host extinction included).

\begin{tabular}{|c|c|c|c|c|c|c|c|}
\hline $\mathrm{SN}$ & Type & $\begin{array}{c}\Delta t_{\mathrm{p}} \\
\text { (days) }\end{array}$ & $\begin{array}{c}\log _{10}\left(\Delta L_{\text {last non-det. }}\right) \\
\left(\log _{10}\left[\mathrm{erg} \mathrm{s}^{-1}\right]\right)\end{array}$ & $\begin{array}{c}\log _{10}\left(L_{\mathrm{p}}\right) \\
\left(\log _{10}\left[\mathrm{erg} \mathrm{s}^{-1}\right]\right)\end{array}$ & $\begin{array}{c}\log _{10}\left(L_{\max }\right) \\
\left(\log _{10}\left[\mathrm{erg} \mathrm{s}^{-1}\right]\right)\end{array}$ & $\begin{array}{c}\Delta M \text { (peak/last non-det.) } \\
(\mathrm{mag})\end{array}$ & $\begin{array}{c}\Delta M \text { (peak/1st det.) } \\
(\text { mag })\end{array}$ \\
\hline $2005 \mathrm{hl}$ & $\mathrm{Ib}$ & $\ldots$ & $\ldots$ & $<42.63$ & 42.67 & 6.08 & 0.10 \\
\hline $2005 \mathrm{hm}$ & $\mathrm{Ib}$ & $<6.8^{*}$ & $<40.35$ & $<41.56$ & 42.04 & 4.23 & 1.21 \\
\hline $2005 \mathrm{kr}$ & Ic-BL & $<2.6$ & $<41.57$ & $<41.95$ & 42.91 & 3.36 & 2.41 \\
\hline $2005 \mathrm{ks}$ & Ic-BL & $<1.8$ & $<41.21$ & $<42.15$ & 42.93 & 4.29 & 1.97 \\
\hline $2005 \mathrm{mn}$ & $\mathrm{Ib}$ & $<8.6$ & $<40.99$ & $<41.80$ & $\ldots$ & $\ldots$ & $\ldots$ \\
\hline $2006 \mathrm{fe}$ & Ic & $\ldots$ & $\ldots$ & $<42.24$ & 42.67 & 4.26 & 1.10 \\
\hline 2006fo & $\mathrm{Ib}$ & $<18.6$ & $<39.90$ & $<42.39$ & 42.56 & 6.65 & 0.42 \\
\hline 14475 & Ic-BL & $<4.4$ & $<41.63$ & $<42.73$ & 43.28 & 4.14 & 1.40 \\
\hline 2006jo & $\mathrm{Ib}$ & $<11.1$ & $<40.99$ & $<42.75$ & 43.00 & 5.03 & 0.62 \\
\hline 2006lc & $\mathrm{Ib}$ & $5.9-17.6$ & $\ldots$ & 40.91 & 42.59 & $\ldots$ & 4.20 \\
\hline $2006 n x$ & Ic-BL & $<2.6$ & $<41.41$ & $<42.83$ & 43.50 & 5.24 & 1.68 \\
\hline $2006 q k$ & Ic & $<1.9$ & $<40.98$ & $<41.18$ & $\ldots$ & $\ldots$ & $\ldots$ \\
\hline $2007 \mathrm{~ms}$ & Ic & $<9.7$ & $<40.51$ & $<41.81$ & 42.48 & 4.93 & 1.67 \\
\hline $2007 \mathrm{nc}$ & $\mathrm{Ib}$ & $<7.3$ & $<41.51$ & $<41.86$ & 42.42 & 2.27 & 1.42 \\
\hline $2007 q v$ & Ic & $<5.4$ & $<41.51$ & $<41.81$ & 42.53 & 2.55 & 1.79 \\
\hline $2007 \mathrm{qx}$ & Ic & $<1.8^{* *}$ & $\ldots$ & $41.63^{* * *}$ & 42.62 & $\ldots$ & 2.47 \\
\hline $2007 \mathrm{sj}$ & Ic & $<3.8$ & $<40.53$ & $<40.78$ & $\ldots$ & $\ldots$ & $\ldots$ \\
\hline
\end{tabular}

Notes. $\Delta t_{\mathrm{p}}$ corresponds to the time interval between last non-detection and first detection in the rest frame, $L_{\mathrm{p}}$ to the luminosity of the $1 \mathrm{st}$ detection. The limit on the luminosity of the last non-detection $\left(\Delta L_{\text {last non-detec. }}\right)$ corresponds to its $1 \sigma$ error. The last two columns $(\Delta M)$ report the difference in magnitude between the peak and the last non-detection limit, and the difference in magnitude between peak and the 1st-detection luminosity. ${ }^{(*)}$ Time interval between the first two epochs; ${ }^{(*)}$ Time interval between the second and the fourth epoch; ${ }^{(* *)}$ Luminosity of the third epoch. 